\title{
Molecular, anatomical, and functional organization of lung interoceptors
}

\author{
Yin Liu ${ }^{1}$, Alex J. Diaz de Arce ${ }^{1}$, Mark A. Krasnow ${ }^{1 *}$ \\ ${ }^{1}$ Department of Biochemistry and Howard Hughes Medical Institute, \\ Stanford University School of Medicine, Stanford, CA, USA.
}

* Lead contact. Correspondence to: krasnow@stanford.edu 


\begin{abstract}
Interoceptors, sensory neurons that monitor internal organs and states, are essential for physiological homeostasis and generating internal perceptions. Here we describe a comprehensive transcriptomic atlas of interoceptors of the mouse lung, defining 10 molecular subtypes that differ in developmental origin, myelination, receptive fields, terminal morphologies, and cell contacts. Each subtype expresses a unique but overlapping combination of sensory receptors that detect diverse physiological and pathological stimuli, and each can signal to distinct sets of lung cells including immune cells, forming a local neuroimmune interaction network. Functional interrogation of two mechanosensory subtypes reveals exquisitely-specific homeostatic roles in breathing, one regulating inspiratory time and the other inspiratory flow. The results suggest that lung interoceptors encode diverse and dynamic sensory information rivaling that of canonical exteroceptors, and this information is used to drive myriad local cellular interactions and enable precision control of breathing, while providing only vague perceptions of organ states.
\end{abstract}




\section{INTRODUCTION}

As we vividly perceive the external world through the canonical five senses, our nervous system also continuously monitors our internal organs and environment including digested nutrients, blood oxygen, and lung volume as well as tissue damage and inflammation (Cameron, 2002; Grundy et al., 2019; Kumar and Prabhakar, 2012; Raybould, 2010; Umans and Liberles, 2018). This internal monitoring is in part carried out by a large population of sensory neurons, dubbed "interoceptors" over a century ago (Sherrington, 1906), whose cell bodies reside in cranial and dorsal root ganglia (Cameron, 2002). Interoceptors are pseudounipolar cells with one axonal branch projecting to the organ where it senses internal signals, and the other projecting to the brainstem or spinal cord where it transmits sensory information to the central nervous system through synapses with second-order neurons. Sensory information transmitted by these neurons is essential for homeostatic regulation of physiological processes such as digestion, circulation, and breathing (Dockray, 2013; Kumada et al., 1990; Widdicombe, 2006), for triggering defense responses such as vomiting and coughing (Canning et al., 2006; Horn, 2014), and for generating interoception, the higher-order brain perception of internal states (Craig, 2002; Quadt et al., 2018).

Although the neurons, sensors, transduction mechanisms and circuitry of the canonical external sensory systems are well understood, much less is known about interoceptors. Classical histological and electrophysiological studies determined their sizes, myelination states and conduction velocities and explored their sensory modalities and adaptation properties. These elegant studies showed that interoceptors of many organs are anatomically, physiologically, and functionally heterogeneous (Brookes et al., 2013; Jensen et al., 2013; Longhurst, 1984; Mazzone and Undem, 2016). But only in the past decade, as molecular markers of specific subsets of 
interoceptors began to be uncovered, could such subpopulations be genetically labeled and manipulated to reveal (and register) their full structure, sensory and electrophysiological properties, and functional outputs (Chang et al., 2015b; Williams et al., 2016). Recent application of single cell RNA-sequencing (scRNA-seq) to the mouse vagal ganglion, a major interoceptor ganglion serving most internal organs, revealed further molecular diversity of interoceptors and enabled anatomical and functional interrogation of several subpopulations targeting the gut and the upper airway (Bai et al., 2019; Kupari et al., 2019; Prescott et al., 2020). However, to decipher the cellular and molecular logic of internal sensing, it is important to elucidate the full set of interoceptor subtypes for each organ and their molecular, anatomical, and functional features. Here, we describe a way to identify and characterize an organ's full set of interoceptors and apply it to the mouse lung, providing a comprehensive analysis of interoceptors across an entire organ.

The lung is extensively innervated by sensory neurons whose cell bodies reside almost exclusively in the vagal ganglia (Figure S1B). These interoceptor neurons (commonly called bronchopulmonary sensory neurons but abbreviated pulmonary sensory neurons, or PSNs, here) monitor inhaled air and its effects on the lung, as well as organ damage and inflammation (Lee and Yu, 2014; Mazzone and Undem, 2016). Sensory information transmitted by PSNs to the brainstem breathing and autonomic centers regulates bronchial tone and respiratory rate and pattern, and it also triggers protective reflexes such as cough in response to inhaled irritants, allergens and pathogens (Canning et al., 2006; Lee and Yu, 2014; Mazzone and Undem, 2016; Widdicombe, 2006). Some of this sensory information is conveyed to higher-order processing centers to generate interoceptions such as the distress of dyspnea (shortness of breath or "air 
hunger") (Nishino et al., 2000; Taguchi et al., 1991; Tetzlaff et al., 1999), the urge to cough (Driessen et al., 2017) and likely the satisfying calm of deep breaths.

Classical electrophysiological studies identified three major PSN subtypes: two types of myelinated fast-conducting (A-fiber) PSNs that both respond to lung inflation but with different adaptation properties (rapidly adapting receptors, RARs; slowly adapting receptors, SARs), and non-myelinated slow-conducting C-fibers that respond to harmful exogenous and endogenous chemicals such as irritants and inflammatory factors (Lee and Pisarri, 2001; Lee and Yu, 2014; Mazzone and Undem, 2016). PSNs have a variety of terminal structures ranging from free-nerve endings to specialized smooth muscle-associated and neuroepithelial body (NEB)-innervating terminals (Brouns et al., 2012), and manipulating different molecular subsets of PSNs causes distinct changes in respiratory physiology such as alterations in bronchial tone, airway secretion, respiratory rate and tidal volume (Chang et al., 2015b; Han et al., 2018; Mazzone and Undem, 2016; Nonomura et al., 2017) (Figure S1A). However, the structure and function of the best characterized PSNs remains provisional because for even the most precisely targeted molecular subpopulations it is uncertain how many subtypes were labeled and manipulated, given that marker specificity across the full set of PSNs is unknown. Indeed, there is likely molecular diversity beyond the classical subtypes because PSNs with other physiological properties (e.g., A $\delta$ high threshold receptors (AHTRs) and deflation receptors (Yu, 2005; Zhang et al., 2006)) and distinct developmental origins (e.g., C-fibers originating from neural crest vs neural placode (Nassenstein et al., 2010)) have been described.

Here we create a comprehensive transcriptomic atlas of mouse PSNs that defines 10 molecular subtypes and their relationship to the classical and more recently studied molecular subpopulations. We assign the developmental origin, cell size, and myelination state of each 
subtype, and develop specific labeling strategies to map their receptive fields, terminal morphologies, and cell contacts in the lung. We describe the sensory repertoires and secreted signals for each subtype as well as the potential recipient cells of many of these signals in the lung, focusing on interactions with resident immune cells. Functional interrogation of two myelinated mechanosensory subtypes reveals their exquisitely specific homeostatic roles in breathing regulation. The results suggest that PSNs encode rich and dynamic lung sensory information that drives myriad local cellular interactions and enables precision control of breathing. 


\section{RESULTS}

\section{Labeling and single cell mRNA profiling of vagal PSNs}

To create a comprehensive gene expression atlas of PSNs, we carried out single-cell RNA sequencing (scRNA-seq) on vagal sensory neurons that innervate the mouse lung (Figure 1A). PSNs were labeled by intratracheally instilling fluorescent wheat germ agglutinin (WGA) into the lung and allowing 4-10 days for WGA uptake and retrograde transport to the cell bodies in the ganglion. This labeled $431+/-48$ neurons per ganglion (mean+/-SD, $n=6$ ganglia), $\sim 15 \%$ of the ganglion's 3000 neurons (Fox et al., 2001); a small number of dorsal root ganglion cells were also labeled (Figure S1B) but not considered further here. Co-instillation of a second WGA conjugated to a different fluorophore labeled an almost identical (98\% overlap) population of vagal ganglion neurons, whereas co-injecting the second WGA into stomach labeled a spatially intermingled but entirely non-overlapping ( $0 \%$ overlap) population (Figure $1 \mathrm{~B}, \mathrm{C})$, indicating that PSN labeling by this method is efficient and specific.

Vagal ganglia labeled in this way were dissociated, and single cell suspensions were visualized by fluorescence microscopy. Labeled PSNs were imaged (for archiving and cell size measurements) and then manually picked and transferred to individual tubes for processing, which is gentler on cells and avoids size filtering by fluorescence-activated cell sorting (FACS). Because PSNs originate from both the neural crest and the neural placode (Mazzone and Undem, 2016), and neural crest-derived neurons are rare ( 7\%, Figure S1D) and did not survive dissociation well (see STAR Methods), some WGA labeling was performed in tdTomato reporter lines that allowed us to distinguish the PSN lineages during cell picking (Wnt1Cre;Ai14/+, neural-crest specific; $C G R P \alpha^{C r e E R T 2 /+} ; A i 14 /+$, neural crest-enriched; Phox $2 b$ Cre;Ai14/+, neural placode specific, Figure S1C, D and Table S1) (Danielian et al., 1998; Scott 
et al., 2011; Song et al., 2012) to ensure a sufficient representation of neural crest lineage neurons in our dataset. Complementary DNA synthesis and amplification of RNA from individual neurons were done by SmartSeq2 protocol, using fewer amplification cycles (14-15) than normal (18-21) to minimize mRNA amplification bias. We recovered enough cDNA for sequencing library construction from 136 labeled cells, roughly one-third of the total PSNs in a ganglion.

Sequencing reads were aligned to the mouse transcriptome (RefSeq), and cells with fewer than 2 million mapped paired-end reads or 10,000 expressed endogenous genes were excluded. We also excluded glial contaminated cells by performing principal component analysis (PCA) using genes enriched in satellite glial cells in peripheral sensory ganglia (Li et al., 2016) (Figure S1E, F) (STAR Methods). The final dataset contained 123 neuronal expression profiles, with an average of 5.4 million total paired-end reads (range 2.4 to 12.4 million) that mapped to an average of 12,500 endogenous genes per cell (range 10,300 14,000 genes). The number of expressed genes detected per cell was $60 \sim 80 \%$ greater than in droplet-based scRNA-seq data of vagal sensory neurons (average 6500 8000 genes per cell) (Bai et al., 2019; Kupari et al., 2019). We examined expression levels of housekeeping genes (Gapdh and $A c t b$ ), vagal sensory neuron marker gene Slc17a6 (Chang et al., 2015b) and fluorescent marker tdTomato in our dataset and detected no drop out and very low variance across all cells (Figure S1G, H). Thus, this vagal PSN scRNA-seq dataset is of high quality and, as we show below, covers all subtypes.

\section{Identification of 10 molecular subtypes of PSNs}

To identify molecularly distinct subtypes, we clustered PSNs based on the similarity of their expression profiles, using a customized iterative strategy modified from one developed for mouse cortical neurons (Tasic et al., 2016) (STAR Methods) (Figure 1D). In each round of 
clustering, neurons were split into two clusters by PCA using genes with highly variable expression levels across the full set, and each obtained cluster was subsequently sub-clustered in a similar manner in the next round; the robustness of each separation was tested by re-clustering using random subsamples $(80 \%)$ of the highly variable genes, and only neurons that stably clustered together were designated as a cluster and promoted to the next round (or accepted as final). Each final cluster was cross-validated with each of the other clusters to confirm robust separation. In this way, we identified eight clusters (PSN1 to PSN8), referred to as PSN subtypes hereafter. We also identified two additional PSN subtypes. PSN9, which separated from PSN8 in the third round of clustering (Figure 1D), was too small a group (two neurons) to be designated a cluster by our algorithm's criteria but was subsequently validated by in situ hybridization as a rare but distinct subtype (Figure S2D). The final subtype we named PSN-H because these neurons are molecular hybrids of PSN2 and PSN3 neurons; in the third round of clustering that distinguished PSN2 and PSN3, PSN-H neurons variably clustered with one or the other depending on the subset of highly variable genes used in the clustering (Figure 1D). They coexpress many of the differentially expressed genes that distinguish PSN2 and PSN3 neurons (Figure 1E), and we did not identify any uniquely expressed genes that distinguish them from PSN2 and 3. Most of the co-expressed genes, including many receptors and channels, displayed a gradual change in expression level across the population (Figure 1E), indicating that PSN-H represents an intermediate functional state between PSN2 and PSN3 neurons.

The traditional classification of PSNs is based in part on their fiber types, which can be distinguished by their cell body diameter and neurofilament heavy chain expression (Lawson and Waddell, 1991). PSN1, 2, 3, and H, which separated as one group (Group I neurons) in the first round of clustering, are composed of small to medium diameter neurons that express low levels 
of Nefh (Figure 1F, G), indicating they are C-fiber neurons. PSN4 to 9 (Group II neurons) are composed of small (PSN4 and 5), medium (PSN8) and large diameter neurons (PSN6, 7 and 9) (Figure 1F). PSN6, 7 and 9 all express high levels of Nefh, indicating they are thickly myelinated (A $\beta$-fiber) neurons. PSN8 neurons express intermediate levels of $N e f h$, suggesting they are myelinated but potentially with thinner myelination (A $\beta$ or A $\delta$-fiber) than PSN6, 7 and 9. PSN4 and 5 express slightly higher levels of Nefh than Group I neurons, so may be thinly myelinated (A $\delta$-fiber) or non-myelinated (C-fiber) (Figure $1 \mathrm{G}$ ). We could also provisionally assign developmental lineages based on the expression of neural placode marker Phox $2 b$ (Dauger et al., 2003): PSN2 to 8 and $\mathrm{H}$ all express high levels so likely arise from neural placode, whereas PSN1 and 9 did not express Phox $2 b$ so likely arise from neural crest (Figure $1 \mathrm{H})$. Thus, the 10 identified molecular PSN subtypes comprise different fiber types and developmental origins. Subtypes also differ in abundance, and each has distinct molecular, anatomical, and functional features that begin to reveal their specific roles in the sensory biology of the lung and breathing, as described below.

\section{PSN subtype markers and abundance in vivo}

To find specific markers for each PSN subtype, we used the single cell differential expression (SCDE) method (Kharchenko et al., 2014) to compare the expression profiles of each subtype with every other subtype individually, then intersected the lists of differentially expressed genes.

This identified 10 to 89 genes enriched in each PSN subtype (except hybrid subtype PSN-H), and 260 genes in total for the 9 subtypes (Figure 2A and S2A). We further selected the best markers for each subtype from these subtype-enriched genes based on their expression specificity and levels (Table S2). Several previously used markers of mouse PSN subpopulations were not among the 260 subtype-enriched genes because they are expressed in multiple PSN subtypes 
(Trpv1, Piezo2, P2ry1, Slc7a7, P2rx2, and P2rx3) (Brouns et al., 2009; Chang et al., 2015b;

Nassenstein et al., 2010; Nonomura et al., 2017). Six extant markers were selectively enriched in single subtypes: PSN1 (Trpa1, Tac1, Mgprx1, Gfra3) (Han et al., 2018; Nassenstein et al., 2008; Nassenstein et al., 2010), PSN2 (Npy2r) (Chang et al., 2015b), and PSN7 (Calb1) (Brouns et al., 2009), but only two (Gfra3 and Calb1) were among the top subtype markers with high specificity and robust expression (Figure S2B). Thus, our comprehensive profiling data identified specific and robust molecular markers that allow identification, manipulation, and functional interrogation of 9 of the 10 PSN molecular subtypes we defined (all except hybrid subtype PSN-H).

We next examined the expression of highly selective and robustly expressed marker genes (Figure 2B) to determine the abundance of each identified PSN subtype in vivo. Single molecule fluorescence in situ hybridization (smFISH) and/or immunostaining of vagal ganglion sections, in which PSNs had been retrogradely labeled with WGA, identified all 10 PSN subtypes, including the hybrid subtype PSN-H (a subset of which are Npy $2 r^{+} H t r 3 b^{+}$) and PSN9 (Phox $2 b^{-}$ Gal $^{-}$or Phox $2 b^{-} \operatorname{Trpv1}^{-}$) (Figure 2B and S2C). Cell counts showed substantial differences in abundance among the subtypes, from $2.5 \%$ ( $\sim 10$ neurons per side) for PSN9 to $15 \sim 20 \%(60 \sim 80$ neurons) for PSN2, 3 and 8 (Figure 2C, D and S2C). These abundance values agree well with our scRNA-seq data, implying minimal cell type bias during manual cell picking (Figure 2C). The summed abundance of all 10 subtypes accounts for virtually all WGA-labeled PSNs (Figure 2D), indicating the 10 subtypes comprise an essentially complete molecular cell atlas of vagal PSNs.

\section{Lung distribution, terminal morphologies, and cell contacts of PSN subtypes}


The lung is composed of a serial branching bronchial tree (the conducting airways) and millions of alveoli, where gas exchange occurs, together with an intricate vascular network. To begin to define the receptive fields and sensory mechanisms of PSN subtypes, we devised genetic labeling strategies with subtype selective genes to map subtype terminal distributions, morphologies, and cell contacts in the lung. Subtype labeling was performed by injecting an adeno-associated virus (AAV) expressing a Cre-dependent reporter (fluorescent protein or alkaline phosphatase, AP) into the vagal ganglia (or instilling them into the lungs) of mice expressing Cre recombinase driven by a gene selectively expressed by the subtype(s) of interest (Figure S3A).

Terminals of Group I neurons were labeled by injecting AAV-CAG-DIO-tdTomato into the vagal ganglia of $\operatorname{Trpv1}{ }^{\mathrm{Cre} /+}$ mice, since Trpv1 is expressed in the vast majority of Group I neurons but rarely in Group II neurons (Figure S2B). TdTomato-labeled axons exhibited simple terminal structures (free-nerve endings) and terminated throughout the bronchial tree and alveoli (Figure 2E, F). To distinguish axons of PSN1 neurons from other Group I neurons, we co-stained lung sections with an antibody against substance P (SP), a neuropeptide encoded by Tacl, which is selectively expressed by PSN1 among Group I neurons (Figure S2B). TdTomato ${ }^{+} \mathrm{SP}^{+}$fibers ramified beneath the airway smooth muscle fibers throughout the conducting airways, with limited penetration of the alveolar region (Figure 2E, F, O, P and S3B). These fibers also terminated on blood vessels, generally external to the vascular smooth muscle layer (Figure S3C and D). Thus, the sensory field of PSN1 neurons is the peribronchial and perivascular mesenchyme. Tdtomato ${ }^{+} \mathrm{SP}^{-}$fibers, presumably from PSN2, $\mathrm{H}$ and 3 neurons, terminated along the conducting airways (Figure 2E), on blood vessels (Figure S3E) and in the alveolar region (Figure 2F). In the alveolar region, these fibers branched extensively (Figure 2F), running mainly 
along the boundaries between alveoli, with rare cases that elaborated on air sacs (Figure 2G, H and S3F). PSN2 neurons (and likely some PSN-H neurons) almost exclusively form alveolar terminals and only rarely terminate on bronchial airways, as shown by whole-mount AP staining of lungs from $\mathrm{Npy} 2 r^{\mathrm{ires}-\mathrm{Cre} /+}$ mice intratracheally instilled with the AP virus (Figure S3G-H) and a prior study that injected a reporter virus into the vagal ganglia of $N p y 2 r^{\text {ires-Cre/+ }}$ mice (Chang et al., 2015b). Thus, the major sensory field of PSN2 neurons is the alveoli, and the tdTomato ${ }^{+} \mathrm{SP}^{-}$ fibers that terminated on conducting airways (observed in $\operatorname{Trpv} 1^{\mathrm{Cre} /+}$ mice) most likely emanated from PSN3 (and some PSN-H) neurons (Figure 2E, O and P).

We also mapped the terminals of two Group II subtypes, PSN6 and PSN7, which we subsequently functionally characterized (see below). We labeled PSN6 neurons by injecting either AAV-CAG-DIO-tdTomato or AAV-Syn-DIO-hM3Dq-mCherry into the vagal ganglia of $M c 4 r^{2 \mathrm{~A}-\mathrm{Cre} /+}$ mice (the latter AAV labeled membrane structures so better revealed terminal morphologies) (Figure 2J) (Garfield et al., 2015). Although Mc4r is also expressed in PSN5 neurons (Figure S3I), fortuitously the AAV preferentially infected and labeled PSN6 neurons (Figure S3J and K). We found that mCherry labeling in the lung was, with rare exception (presumably PSN5 neurons), confined to multi-branched, "leaf" shape terminals intercalating between airway smooth muscle fibers located just beneath (basal to) the airway epithelium (Figure $2 \mathrm{~J}$ and $\mathrm{K}$ ). These resemble the smooth muscle-associated terminals observed in humans and other species (Brouns et al., 2006; Larshell and Dow, 1933). Interestingly, PSN6 terminals were not uniformly distributed across airway smooth muscle: a large majority of terminals localized to smooth muscle at bronchial branch points (Figure 2I and S3L), and whole-mount staining of AP-labeled PSN6 fibers showed them terminating mainly on primary and secondary bronchial branches and concentrated centrally in lobes (Figure S3O and R). We conclude that the 
PSN6 sensory field is airway smooth muscle cells at branch points, mainly in the middle region of the bronchial tree (Figure $2 \mathrm{O}$ and $\mathrm{P}$ ). It appeared that most of the individual labeled fibers form a small number of terminals (1 4) within close proximity (Figure S3S), suggesting a restricted receptive field for individual neurons.

PSN7 neurons were labeled by injecting AAV-Syn-DIO-hM3Dq-mCherry into the vagal ganglia of $\mathrm{Calbl}^{2 \mathrm{~A}-\mathrm{dgCre} /+}$ mice, in which any expressed destabilized Cre protein (dgCre) is rapidly degraded unless stabilized by trimethoprim (TMP) (Evans et al., 2017). TMP delivery $\left(300 \mu \mathrm{g} / \mathrm{g}\right.$ body weight) two days after viral injection specifically labeled Calb $1^{+}$neurons among WGA-labeled PSNs (Figure S3M and N). All mCherry ${ }^{+}$fibers terminated on neuroepithelial bodies (NEBs) (Figure 2L), clusters of neurosensory epithelial cells (neuroendocrine cells) located at bronchial branch points (Lauweryns et al., 1974), where Calb1-stained neurites have previously been observed in immunostained lung sections (Brouns et al., 2009). Labeled PSN7 axons penetrated the basement membrane of the epithelial layer of the NEBs, coursed between neuroendocrine cells, and terminated in an elaborate "waffle" shape covering their apical surface, facing the airway lumen (Figure 2M-N). Whole-mount staining of AP-labeled PSN7 axons showed that they also terminate on major bronchial branches and were enriched in the proximal regions of the lobes (Figure S3P and R). We conclude that the PSN7 sensory field is the airway surface of neuroendocrine cells at bronchial branch points, mainly in the proximal region of the bronchial tree (Figure S3Q and S). A substantial fraction of labeled fibers forms multiple terminals, which can be distributed through multiple branch points (Figure S3T), suggesting a broader receptive field than PSN6 neurons. Interestingly, some PSN4 terminals also map to NEBs, although they have a distinct terminal morphology and often target different NEBs (A.J.D., Y.L., and M.A.K., in preparation). 
Thus, molecularly distinct subtypes of both Group I and Group II PSN subtypes have different lung receptive fields, and their terminals have different morphologies and target cells.

\section{Subtype diversity in physiological sensing and neuronal signaling}

PSNs can be activated or modulated by a variety of stimuli including chemical irritants, heat, mechanical deformation, and changes in ambient gas composition (Mazzone and Undem, 2016; Ruan et al., 2005; Schelegle and Green, 2001; Yu, 2005). To predict the sensory functions of each PSN subtype, we screened the expression of genes with known functions in external and internal sensing and their family members, including mechano- and thermo-receptors, and receptors for volatile and non-volatile chemicals (Figure 3A and Table S3). This analysis identified subtypes that mediate known sensory functions of PSNs from the classical studies and putative sensors, and also uncovered potential new sensory functions and unexpected sensors. It revealed that every PSN subtype likely detects multiple types of stimuli via different combinations of sensors, and conversely most stimuli can be detected by more than one PSN subtype.

Hyperthermia and chemical irritants such as acids, ozone and toxic aldehydes can stimulate pulmonary C-fibers or potentiate their responses to other stimuli (Lee et al., 2013; Lin et al., 2009; Nassenstein et al., 2008; Ruan et al., 2005; Taylor-Clark and Undem, 2010). Many receptors detecting these stimuli are expressed by PSNs, and some of them are not limited to the Group I (C-fiber) subtypes (e.g., thermal receptors $\operatorname{Trpv} 2$ and $\operatorname{Trpm} 3$, acid-sensing channels Asic1-3), suggesting that these signals may also modulate the activity of A-fiber subtypes (Figure 3A). The polymodal irritant receptor Trpal, which can be activated by many chemical irritants including aldehydes present in cigarette smoke (CS) and by cold temperature (Bautista et al., 2006; Fajardo et al., 2008), is selectively expressed in PSN1 and PSN2 (and a subset of PSN-H) 
neurons, but not in the other Group I subtype PSN3. Hence, PSN1 and PSN2 subtypes represent classical irritant-sensing pulmonary C-fibers (Nassenstein et al., 2008). Interestingly, PSN1 also expresses the highest levels of nicotinic acetylcholine receptors that detect nicotine in CS and exclusively expresses the acid sensing GPCR Gpr68, which has a high activation threshold ( $\sim$ pH6.8) close to physiological pH (Ludwig et al., 2003). Given that PSN1 terminates predominately on conducting airways where inhaled air enters the lung, this subtype may be especially important for mediating protective reflexes in response to irritants.

Six of the 10 subtypes (plus a subset of PSN-H neurons) express mechanosensitive Piezo channels, indicating they are putative mechanoreceptors. These include all Group II subtypes (except PSN4) expressing Piezo2, and the Trpa1- Group I subtype PSN3 (and a subset of PSN-H neurons) expressing Piezol; PSN8 is the only subtype that co-expresses both Piezo channels. PSN5, in addition to Piezo2, also expresses Tmc3, a family member of the auditory hair cell mechanotransduction channels Tmc1/2 (Pan et al., 2013), along with the other two components of the mechanotransduction complex, Tmie and Lhfpl5 (Xiong et al., 2012; Zhao et al., 2014). The different mechanosensors and their combinations expressed by individual subtypes may contribute to their detection of different types of mechanical stimuli, such as inflation vs. lung compliance, or to different response thresholds (Ma et al., 2003; Mazzone and Undem, 2016). Additional polymodal mechanosensitive channels, such as two-pore potassium channels and acid sensing ion channels, are also expressed in multiple subtypes (Table S3), including PSN1 and PSN2, suggesting the irritant-sensing subtypes may also be modulated by mechanical stimuli.

Hypercapnia (high $\mathrm{CO}_{2}$ ) has been shown to increase the activity of pulmonary C-fibers and suppress the activity of pulmonary mechanoreceptors, the latter depending on carbonic anhydrase (CA) (Lin et al., 2005; Matsumoto et al., 1996; Schelegle, 2003). Multiple CA genes 
are expressed by PSNs, and several (Car2, $\operatorname{Car} 8, \operatorname{Car} 9$, $\operatorname{Car} 13)$ are selectively enriched in subsets of PSN subtypes. Car2, which mediates olfactory detection of $\mathrm{CO}_{2}$ (Hu et al., 2007), has the highest expression levels and is selective for two putative mechanosensory subtypes (PSN6 and 7) (Figure 3A). As we show below, these two subtypes control eupneic breathing, suggesting that the control mechanisms may be inactivated under hypercapnia by Car2-mediated suppression of their mechanosensory functions.

We also uncovered the expression of genes that mediate sensory functions previously unknown in PSNs, including light-sensitive non-visual opsins Opn3 and Opn4, the latter selectively expressed in PSN6 and 7. Several olfactory and vomeronasal receptors were also detected, such as Olfr1033, Olfr 78 and Olfr920, and they are differentially expressed across PSN subtypes. Among them, Olfr 78, enriched in PSN3 and 4, is of particular interest because it can respond to lactate and mediate the acute response to hypoxemia in carotid bodies (Chang et al., 2015a). PSN4 also expresses Kcnk3/TASK-1, another acute hypoxia response mediator (Trapp et al., 2008) and high levels of genes in the HIF chronic hypoxia response pathway (Arnt2/Hif2b, Egln3/Phd3). Together, these suggest that PSN4 could be a novel pulmonary hypoxia sensor (A.J.D., Y.L., and M.A.K., in preparation).

In addition to detecting physiological stimuli, PSNs also express receptors for cellular components specific to bacteria (Tlr4, Tlr5, Nod1, Naip1 and Naip2) with subtype-specific patterns (Figure 3B). Notably, PSN7 neurons, which terminate on the apical side of NEBs, selectively express both Tlr5, which detects bacterial flagellin (Hayashi et al., 2001), and Naip2, which detects bacterial T3SS inner rod protein (Zhao et al., 2011), suggesting that this subtype might sense bacterial infections. We also found that mouse PSNs express at least 22 genes that enable entry of 10 types of respiratory viruses in human, including Uvrag for influenza (Pirooz et 
al., 2014), Igflr for respiratory syncytial virus (RSV) (Griffiths et al., 2020) and Pvrl4 for measles (Mühlebach et al., 2011) (Figure S4C). PSNs also express intracellular RNA and DNA sensors (Ddx58,Dhx58,Ddx41, Lrrfip1 and Aim2) that can recognize viral nucleic acids and activate downstream signaling (Figure S4C).

All PSNs project to the brainstem, transmitting sensory information to physiological control circuits. To identify the neuronal signals used by each PSN subtype, we analyzed expression of genes encoding neurotransmitter synthesis and secretion machineries and neuropeptides. As expected from previous studies, all are glutamatergic (all express Slc17a6 and a subset Slc17a7), but we also identified two novel PSN neurotransmitters: acetylcholine in PSN4 (expresses Chat and Slc18a3) and dopamine in PSN5 (expresses $T h$ and $D d c$, but not $D b h$ ). We also found that nitric oxide synthase genes (Nos1 and Nos3) are widely expressed across PSN subtypes, with PSN1 expressing neuronal NOS (Nos1) at the highest level, suggesting the use of NO. A surprisingly large number of neuropeptide genes ( $77 \%, 57$ out of 74 known genes) were detected in PSNs (Table S4) and 23 of these were expressed by considerable number of PSNs (in $>50 \%$ PSNs in at least one subtype) and exhibited subtype-selective expression patterns including several that are specific for a single subtype (Gal in PSN1, Penk, Uts2b and Ghrh in PSN6, and Pthlh in PSN7) (Figure 3C).

Together the data indicates that each PSN subtype expresses a different but overlapping combination of physiological sensors and potentially responds to multiple modes of stimuli, and they use diverse neurotransmitters and neuropeptides for intercellular communication (Figure 3D and S4B).

\section{PSN sensing and signaling inflammation}


In addition to physiological sensors, PSNs express a remarkable variety of receptors for inflammatory signals including purines (e.g., ATP and adenosine), biogenic amines (serotonin and histamine), lipids (e.g., lysophosphatidic acid, sphingosine-1-phosphate, and prostaglandins), proteases (e.g., thrombin and trypsin) (Figure 4A) and cytokines (e.g., interleukins, chemokines and tumor necrosis factors) (Figures 4B, S5). For many inflammatory signals, the receptors are expressed across all PSN subtypes (e.g., ATP, prostaglandins and CXCL2), although with different combination of receptor isoforms. This implies widespread influence of inflammation on PSNs. However, other signals target specific subtypes (e.g., lysophosphatidic acid on PSN1, 2, and H and TNF- $\alpha$ on PSN2, H, 3, 7, 8, and 9) or even a single subtype (e.g., OSM on PSN1). Thus, each PSN subtype appears to detect multiple, largely overlapping but distinct combinations of inflammatory signals, as for their detection of physiological signals.

To determine the local sources of the inflammatory signals, we surveyed our comprehensive mouse lung cell atlas (Tabula Muris, 2018; 2020; Travaglini et al., 2020) for expression of cognate ligands for the identified PSN inflammatory receptors. We focused on cytokine signals because the ligand sources can be unambiguously identified by mRNA expression profiles. This predicts an interaction network in which a variety of lung cell types can signal to PSNs: nonimmune cells use primarily TGFB2/3 and TNFSF $10 / 12 / 13 \mathrm{~b}$, and immune cells use interleukins (IL4, OSM) and chemokines (CXCL1/2) plus TGFB1 and TNFSF12/13b, with neutrophils an especially rich source of CXCL2, basophils of IL4, and both granulocytes of OSM (Figure 4B, C and S5). These cytokines are presumably stored under healthy conditions and released on infection or injury, providing early disease signals to PSNs.

The lung cell atlas also allowed us to predict reciprocal signaling interactions, such as PSN signals back to the immune cells. Besides the neuropeptides that modulate immune cell 
behaviors implicated in previous studies (e.g., CGRP and VIP) (Baral et al., 2018; Talbot et al., 2015) (Figure 3C), we found that PSNs also express many cytokines, several in distinct patterns among the PSN subtypes such as $I l 18, C s f 1$ and $C x c l 16$. Their receptors are likewise differentially expressed across immune cell types (e.g., Csflr mainly in monocytes and interstitial macrophages and $\mathrm{Cxcr} 6$ in $\mathrm{CD} 8^{+} \mathrm{T}$ cells); collectively they are distributed across all types of immune cells, including both innate and adaptive immune cells (Figure 4B, C and S5 and S6). This bidirectional cytokine signaling between PSNs and immune cells suggests that PSNs potentially bridge different types of immune cells in an immune response cascade or amplify the response in certain immune cells. For example, basophils release IL4 upon activation (Kubo, 2017), which can act on PSN1 and 2. These PSNs potentially release CCL2 that signals to monocytes and dendritic cells, as well as IL18 that signals back to basophils (Figure 4B, C and S6). Indeed, IL4 has been shown to induce CCL2 release from bone-marrow derived endothelial progenitor cells (Baba et al., 2020), and similar mechanism may exist in PSNs.

In sum, our analysis reveals a surprisingly broad and intricate neuro-immune interaction network in the lung, highlighting the role of PSNs in recognizing, coordinating, and amplifying inflammatory responses.

\section{PSN6 neurons limit tidal volume by regulating inspiratory time}

We further leveraged our PSN molecular cell atlas to develop strategies to target specific PSN subtypes to assess their functions in breathing regulation. A variety of approaches have been used historically to assess PSN functions, including vagal cooling, pharmacological activation, inhibition of differentially-expressed receptors, and, most recently, optogenetic activation and conditional gene ablation (Chang et al., 2015b; Han et al., 2018; Lee and Yu, 2014; Mazzone and Undem, 2016; Nonomura et al., 2017). However, with our new appreciation of PSN 
diversity we now recognize that previous manipulations, with rare exceptions (see Discussion), targeted multiple PSN subtypes, complicating their interpretation. Here we selectively targeted PSN6 and PSN7, two myelinated subtypes that both express mechanosensitive channel Piezo2 but have distinct receptive fields and terminal morphologies. The results below reveal that each subtype regulates a distinct, highly specific aspect of breathing.

To explore PSN6 function, we selectively eliminated them using the diphtheria toxin (DT)/ DT receptor (DTR) cell ablation system. DTR was expressed in PSN6 neurons by bilaterally injecting AAV-CBA-DIO-DTR-GFP, which expresses a Cre-dependent DTR-GFP fusion transgene (Azim et al., 2014), into the vagal ganglia of mice carrying the PSN6-selective Credriver $M c 4 r^{2 \mathrm{~A}-\mathrm{Cre}}$ (PSN6-DTR mice). Three weeks after viral injection, DTR-GFP was detected in $35-55 \%$ of PSN6 neurons $\left(\mathrm{Lmcdl}^{+} \mathrm{WGA}^{+}\right)$(Figure S7A, B), and three days after subsequent systemic delivery of DT to induce apoptosis of DTR-GFP expressing cells, no DTR-GFP expressing PSNs were detected in the ganglia (Figure S7C, D). To assess the impact of PSN6 loss on breathing, we first performed whole-body plethysmography recordings on freely moving control (wildtype mice injected with the same virus) and PSN6-DTR mice 1 day before and 3 days after DT injection (Figure 5A), focusing the analysis on eupneic ("quiet") breaths computationally selected based on their regular breathing pattern (STAR Methods) (Figure S7E, F). We found that after DT injection the tidal volume (TV) of eupneic breaths in PSN6-DTR mice was increased by $\sim 11 \%$ over control mice (Figure $5 \mathrm{~B}, \mathrm{C}$ ). Inspiratory time (Ti) was extended to a similar degree (Figure 5D-F), accounting for the entire increase in tidal volume. The effect was specific for inspiratory time because inspiratory flow, the other determinant of tidal volume, was not significantly altered (Figure 5G). Expiratory time (Te) showed a similar increase, presumably secondary to the increase in tidal volume (Figure S7G). Despite increased 
tidal volume, minute ventilation remained normal because of a compensatory decrease in respiratory frequency (Figure S7H, I). These results indicate that PSN6 neurons are required for setting the eupneic tidal volume, and they appear to do so by regulating inspiratory time.

The Hering-Breuer inflation reflex, described $\sim 150$ years ago, is a reflex triggered by lung inflation to stop inspiration and prolong expiration, leading to a decrease in respiratory rate or transient apnea (Ullmann, 1970; Widdicombe, 2006). This reflex was traditionally believed to be mediated by sensory neurons terminating on airway smooth muscles (Coleridge and Coleridge, 2011), although recent studies suggest potential involvement of NEB-innervating neurons (Chang et al., 2015b; Nonomura et al., 2017). To determine if PSN6 neurons, which terminate on airway smooth muscle (Figure 2I-K), detect inflation and mediate this reflex, we used an inflation challenge assay (modified from Zhang et al., 2006) in which urethane-anesthetized, open-chest mice were mechanically ventilated with sustained inflations introduced up to defined target pressures, and the impact of each sustained inflation on inspiratory motor activity was measured by concurrent electromyogram (EMG) recording of the diaphragm, an inspiratory muscle (Figure 5H). In wildtype control mice (with viral and DT injections), the frequency of diaphragm activity bursts (i.e., respiratory rate) decreased as the sustained inflation pressure increased, as expected for animals with an intact Hering-Breuer reflex (Figure 5I and S6J). The duration of individual bursts (inspiratory time) declined along with the frequency (Figure 5J, K and S6J), showing that lung inflation shortens inspiratory time in mice as in other species (Clark and von Euler, 1972). In PSN6-ablated mice, the pressure-dependent decrease in inspiratory rate was normal (Figures S7J), suggesting the classical Hering-Breuer inflation reflex was intact. However, the effect of inflation pressure on inspiratory time was diminished (Figure $5 \mathrm{~J}$ and $\mathrm{K}$ ), consistent with our observations from the plethysmography recordings. Interestingly, the 
diminution was only evident during inspiratory holding at 6 and $8 \mathrm{cmH}_{2} \mathrm{O}$, around the target pressure of normal mechanical ventilation $\left(\sim 7 \mathrm{cmH}_{2} \mathrm{O}\right)$ (Figure $\left.\mathrm{S} 7 \mathrm{~K}\right)$, but not at higher levels of inflation (Figure 5J, K). We conclude that PSN6 neurons function during eupneic breathing to specifically regulate tidal volume (and not respiratory rate), and they do so by detecting lung expansion with a low threshold and limiting inspiratory time.

\section{PSN7 neurons limit tidal volume by regulating inspiratory flow}

PSN7 neurons are also myelinated and express mechanosensitive channel Piezo2, but unlike PSN6 neurons they form "waffle" shape terminals on NEBs located at bronchial branch points (Figure 2L-N). To probe their physiological function, we selectively ablated these neurons by injecting AAV-CMV-DIO-DTR-GFP into the vagal ganglia of PSN7-specific Calb1 2A-dgCre/+ mice (PSN7-DTR mice). Three weeks after viral infection and TMP treatment, DTR-GFP was detected in $55-70 \%$ of PSN7 neurons $\left(\mathrm{Calb1}^{+} \mathrm{WGA}^{+}\right)$(Figure S7L, M), and three days after subsequent systemic delivery of DT, no DTR-GFP expressing PSNs were detected in the ganglia, with a single exception (Figure S7N, O). Plethysmography recordings showed that PSN7 ablation increased tidal volume of eupneic breaths by $\sim 15 \%$ over control mice, similar to the effect of PSN6 ablation described above (Figure 6A). However, instead of an increase in inspiratory time as we observed after PSN6 ablation, PSN7 ablation increased peak inspiratory flow by $\sim 13 \%$ over control mice (Figure $6 \mathrm{~B}-\mathrm{E}$ ). The effect was specific as peak expiratory flow was not significantly altered (Figure S7P). Expiratory time and respiratory frequency were unchanged, and minute ventilation showed a slight increase (trending) (Figure S7Q-S). In the inflation challenge assay, there was no attenuation of the effect of inflation on either inspiratory time or respiratory rate (Figure 6F and S7T). Thus, PSN7 neurons do not mediate the Hering- 
bioRxiv preprint doi: https://doi.org/10.1101/2021.11.10.468116; this version posted November 13,2021 . The copyright holder for this preprint (which was not certified by peer review) is the author/funder. All rights reserved. No reuse allowed without permission.

Liu et al., p. 24

Breuer inflation reflex. We conclude that PSN7 neurons are required for setting eupneic tidal volume, but unlike PSN6 neurons, they do so by regulating inspiratory flow. 


\section{DISCUSSION}

Combining organ-specific retrograde labeling and single cell RNA-sequencing, we have provided a comprehensive, high-quality molecular cell atlas of vagal sensory neurons innervating the mouse lung. This defined 10 molecular PSN subtypes, triple the number inferred from classical physiological studies and likely comprising the full set. From our analysis of PSNs' development, myelination, and sensory and signaling gene profiles, our mapping of their terminals elucidating their receptive fields and sites, and our initial characterization of their physiological functions, we can integrate our atlas with prior studies of PSNs (Figure 7A) and begin to assign specific functions to individual subtypes and speculate on the organization and evolution of interoceptive systems.

PSN1 and PSN2, the two typical irritant-sensing C-fiber subtypes both form free nerve endings terminating beneath the epithelium and smooth muscle, where they presumably detect chemical signals, but differ in developmental lineage (PSN1, neural crest; PSN2, neural placode) and receptive field (conducting airways vs. alveoli) (Figure 2E-H). They likely also differ in the physiological responses elicited by their activation. Activating PSN1 neurons with an agonist (Bam8-22) for Mrgprx1 (also named Mrgprc11 in mouse), specific to PSN1 (Figure S2B), leads to reflex bronchoconstriction (Han et al., 2018), whereas activating PSN2 neurons using optogenetics targeting a subset of vagal sensory neurons expressing $N p y 2 r$, which is selectively expressed by PSN2 (and a subset of PSN-H) (Figure S2B), causes rapid and shallow breathing (Chang et al., 2015b). Thus, the signals for receptors expressed by both subtypes (e.g., aldehydes in polluted air and inflammatory signal lysophosphatidic acid) would likely trigger distinct physiological responses in different lung compartments (conducting airways vs. alveoli). 
PSN3 is a newly identified pulmonary C-fiber subtype with similar terminal structures, locations, and distributions as PSN1 (Figure 2E and F). But unlike PSN1, this subtype does not express irritant receptor Trpal but expresses Piezol, so is presumably mechanosensitive (Figure 3A). Pulmonary C-fibers are not activated by normal ventilation (Zhang et al., 2006), hence PSN3 neurons likely detect inflation or some other mechanical stimulus outside the physiological range. PSN3 neurons also express high levels of $H$ tr $3 a$ and $H t r 3 b$, ionotropic channels for serotonin that can activate pulmonary C-fibers leading to apnea (Hsu et al., 2019; Moreira et al., 2007). Thus, PSN3 neurons could mediate a reflex cessation in breathing (apnea) in response to an extreme mechanical stimulus such as lung overinflation. The other newly identified C-fiber subtype, PSN-H, is a curious molecular hybrid between PSN3 and the alveolar irritant detector PSN2. We did not identify any expressed genes unique to PSN-H, rather PSN-H neurons comprise a continuous molecular gradient between PSN2 and PSN3 identities (Figure 1E), reminiscent of a couple of continuously heterogeneous neuronal populations recently observed in the brain (Kim et al., 2020; Stanley et al., 2020). It will be important to determine the distribution and dynamics of PSN-H neurons in vivo to see if they form a spatial or functional gradient or represent dynamic intermediates between plastic PSN2 and PSN3 fates.

The other six PSN subtypes (Group II) include five putative mechanoreceptor subtypes (PSN5-9), which are mostly myelinated, fast conducting neurons. The ones focused on here, PSN6 and 7, displayed specific mechanosensory functions in regulating eupneic breathing. PSN6 neurons fit the anatomical and functional expectations for SARs. Their terminals target bronchial smooth muscle so presumably sense smooth muscle stretch during inflation (Schelegle and Green, 2001). They are required to limit inspiratory time during eupneic breathing thus are likely activated during inspiration to provide homeostatic feedback on lung volume. PSN7 neurons, by 
contrast, terminate atop NEBs on the luminal (apical) side at bronchial branch points and limit inspiratory airflow. These anatomical and functional features suggest that PSN7 neurons could sense airflow or flow-associated shear stress, similar to endothelial cells at branch points of blood vessels (Chistiakov et al., 2017), and thus function as a homeostatic feedback regulator of inspiratory airflow. PSN7 neurons are likely RARs because unlike volume-detecting SARs, their activity is expected to diminish during sustained inflation, when airflow drops to zero. The different physiological outputs generated by PSN6 and PSN7 suggest that they synapse with separate populations of second-order neurons in the NTS, which in turn connect to different output circuits (Figure 7B). One limitation of our functional interrogation experiments is that the ablations were not restricted to interoceptors of the lung. Although it seems unlikely that interoceptors of other organs mediate the same function, new strategies that combine lung and subtype-specific targeting should be developed to confirm these conclusions.

We suspect that the other Group II mechanoreceptor subtypes (PSN5, 8 and 9) have similarly specific roles in breathing regulation, for example detecting other breathing-associated mechanical modalities distinct from those detected by PSN6 or 7, or the same modalities but at different ranges or thresholds. For instance, the effect of PSN6 loss was observed only at low levels of lung inflation, suggesting that one of the other mechanoreceptor subtypes could serve to limit inspiratory time and lung volume at higher inflation levels, such as during vigorous exercise. Also, PSN6 neurons only regulate inspiratory time, indicating that other subtype(s) sense the same modality (airway stretch or volume) but with a different output -- regulation of respiratory rate. In addition to the surprising plethora and diversity of mechanosensory PSN subtypes, we also discovered a unique and novel subtype (PSN4) that potentially functions as an airway hypoxia sensor to be described elsewhere (A.J.D., Y.L., and M.A.K., in preparation). 
Beyond what we consider the dominant sensing function of each PSN subtype under physiological conditions described above, our sensory repertoire analysis revealed that each subtype is also equipped to detect multiple other types of external and internal stimuli, including inflammatory signals and pathogens. This suggests that vastly different types of sensory information can be transmitted by the same PSNs. Some diverse stimuli may independently activate the same subtype and elicit the same physiological response, e.g., both hot air and nicotine induce bronchoconstriction (Lee et al., 2018; Lin et al., 2009; Ruan et al., 2005). Other stimuli could be integrated, one potentiating or suppressing the PSNs' response to another, e.g., prostaglandins heighten the sensitivity of pulmonary C-fibers to capsaicin (Kwong and Lee, 2002) and hypercapnia suppresses the activity of pulmonary mechanoreceptors (Schelegle and Green, 2001). Because subtypes can exhibit distinct firing patterns under different conditions, e.g., breathing-associated mechanical stimuli generate oscillatory firing whereas chemical stimuli cause continuous firing (Zhang et al., 2006), there could also be sensory coding within a neuron if different firing patterns activate different cells and output pathways. Some of the inflammatory or pathogenic signals may not directly alter the electrical activity of PSNs, but rather induce transcriptional changes to alter their sensory repertoire or responsiveness (Dinh et al., 2005; Verzele et al., 2021; Zhang et al., 2008).

Our analysis of the expression of secreted signals also highlights the diversity of PSN subtypes. These signaling molecules mediate PSN functions not only through their central projections to the NTS where they regulate respiration, but also through local release in the lung. It appears that local neuro-immune interactions are potentially much more extensive and elaborate than previously thought: in addition to immune-influencing neuropeptides, PSNs are also a source of many cytokines that can act on different immune cells and form local signaling 
circuits (Figure 4 and S5). Some of these cytokines such as IL18 (asthma, COPD) and IL6 (COVID-19) are already implicated in serious lung diseases (Kawayama et al., 2012; McGonagle et al., 2020). Moreover, the interactions are not limited to Trpv1-expressing small diameter neurons, the focus of previous investigations of neuro-immune interactions in peripheral tissues (Cohen et al., 2020; Jain et al., 2020). We find that all PSN subtypes are likely involved and in different ways, including prominent roles of two medium to large diameter putative mechanoreceptor subtypes (PSN8 and 9) and the inflammatory receptors and cytokines they selectively express (SIpr1, Il17rc, Csf1 and Cxcl1) (Figure 4A-C and S5). Guided by predictions from the PSN expression profiles and initial characterization of the 10 subtypes, it is important to fully define experimentally what each PSN subtype senses, how these sensory inputs are integrated, and how that influences the local and central output signals and their impacts, in both health and disease.

Our comprehensive molecular atlas of lung interoceptors invites comparison of an internal sensory system to the well-studied exteroceptive sensory systems for vision, hearing, smell, taste, touch, and other cutaneous sensations (thermal, pain, itch). Each of the canonical exteroceptive systems comprises diverse primary sensory neurons (or non-neuronal cells), which express different receptors (e.g., opsins, olfactory receptors), or are arrayed in different locations (e.g., hair cells along the cochlea), or form different types of terminals (e.g., touch sensory end organs). These neurons/cells transduce and propagate sensory stimuli with different properties to the brain, creating a vivid conscious picture of the external world. Although the diversity of PSNs does not approach that of olfactory sensory neurons (>1000 olfactory receptor genes in mice, one receptor expressed in each neuron), they rival or exceed that of the other exteroceptive sensory systems. The closest parallel are the cutaneous mechanoreceptors that mediate touch and 
mechanical pain. The number of molecularly distinct pulmonary (7 putative subtypes) and cutaneous mechanoreceptors (at least 4 low-threshold subtypes plus high-threshold subtype(s)) (Sharma et al., 2020) are similar. For both populations, molecular subtypes are associated with diverse anatomical (different terminals at different locations), physiological (various conduction velocities and adaptation properties) and functional (tuned to different types of mechanical stimuli, such as airway stretch and airflow in lung vs. hair deflection and stroking of skin) properties (Abraira and Ginty, 2013) (Figure 7C). These suggest that pulmonary mechanoreceptors provide a rich and dynamic description of changes in internal tissue structure and associated mechanical forces in the lung, similar to what cutaneous mechanoreceptors provide for the skin albeit with far less spatial acuity owing to orders of magnitude fewer PSNs of each subtype (a few dozen to about a hundred neurons) compared to cutaneous subtypes (thousands to tens of thousands of neurons). A key difference between the two systems is the consequences of activating the primary sensory neurons. Cutaneous sensory neurons provide nuanced perceptions of the external world, such as tactile quality and intensity, and only extreme stimuli induce reflex behaviors like withdrawal. In contrast, information conveyed by PSNs seems mainly used for tuning respiratory functions through feedback circuits in the brainstem and local interactions with lung cells, and apparently only limited information reaches consciousness as our perception of the lung is typically vague (Cervero, 1990) (Figure 7C). It will be fascinating but challenging to elucidate the integration of sensory information from the 10 PSN subtypes and map the rest of the brain circuits that mediate autonomic reflexes and those underlying higher order perceptions of lung states, such as the distress of dyspnea and the satisfying calm of deep breaths. 
Since each internal organ has highly specific functions and is exposed to a unique environment, it will be important to use ours or similar approaches to molecularly define the full set of interoceptors for all organs (Bai et al., 2019; Hockley et al., 2019; Mazzone et al., 2020) and compare the subtype composition and features of each subtype between organs. It seems likely that all organs will include at least one subtype of danger/damage-sensing C-fiber plus varying numbers and diversity of sensory subtypes serving in physiological feedback circuits as in lung but tailored during evolution to the function of each organ (e.g., nutrient and osmolarity sensors in the gut and the liver (Jensen et al., 2013; Waise et al., 2018) and mechanoreceptors in the heart and vessels (Kumada et al., 1990; Longhurst, 1984)). Myelinated subtypes are likely reserved for functions in which rapid feedback with high temporal fidelity is critical (Suminaite et al., 2019), such as in breathing and cardiac regulation. It will be interesting to see if organs with similarly vague or no perception under physiological conditions, like the heart and liver, are nevertheless equipped with equally rich sets of interoceptors. Moreover, integrating the expression profiles of interoceptors and resident cells of other organs and comparing the interactomes of different organs should reveal both general interactions throughout the body and ones specific for a particular organ. Such studies, ideally carried out in a variety of species including human, will lay the groundwork for a new understanding of interoception and autonomic physiology, their evolution and their contributions to physical and mental health and disease. 


\section{ACKNOWLEDGEMENTS}

We thank current and former members of the Krasnow lab for experimental support and comments on the manuscript, especially Joe Ouadah for help on scRNA-seq experiment, Kyle Travaglini for organizing and sharing scRNA-seq data of the mouse lung, Issac Domenech (Stanford Summer Research Program) and Andrea Yung for improving the smFISH protocol on vagal ganglion sections, and Christin Kuo for discussions. We thank Zachary Knight, Nirao Shah and Eiman Azim for reagent sharing. We thank Ben Passarelli, Stanford Stem Cell Institute Genome Center and Stanford Functional Genomic Facility for help on scRNA-seq experiments and following analysis. This work was supported by HHMI, Damon Runyon postdoctoral fellowship (Y.L.), Stanford Child Health postdoctoral fellowship (Y.L.), and NHLBI (F31HL137406) (A.J.D.). M.A.K. is a HHMI investigator.

\section{AUTHOR CONTRIBUTIONS}

Y.L. performed all the experiments with help from A.J.D. on viral production and smFISH experiments. Y.L. and M.A.K conceived and designed the experiments, interpreted data, and wrote the manuscript. All authors edited the manuscript. 


\section{REFERENCES}

Abraira, V.E., and Ginty, D.D. (2013). The sensory neurons of touch. Neuron 79, 618-639. 10.1016/j.neuron.2013.07.051.

Azim, E., Jiang, J., Alstermark, B., and Jessell, T.M. (2014). Skilled reaching relies on a V2a propriospinal internal copy circuit. Nature 508, 357-363. 10.1038/nature13021.

Baba, T., Miyazaki, D., Inata, K., Uotani, R., Miyake, H., Sasaki, S.I., Shimizu, Y., Inoue, Y., and Nakamura, K. (2020). Role of IL-4 in bone marrow driven dysregulated angiogenesis and age-related macular degeneration. Elife 9, e54257. 10.7554/eLife.54257.

Bai, L., Mesgarzadeh, S., Ramesh, K.S., Huey, E.L., Liu, Y., Gray, L.A., Aitken, T.J., Chen, Y., Beutler, L.R., Ahn, J.S., et al. (2019). Genetic identification of vagal sensory neurons that control feeding. Cell 179, 1129-1143 e1123. 10.1016/j.cell.2019.10.031.

Baral, P., Umans, B.D., Li, L., Wallrapp, A., Bist, M., Kirschbaum, T., Wei, Y., Zhou, Y., Kuchroo, V.K., Burkett, P.R., et al. (2018). Nociceptor sensory neurons suppress neutrophil and gammadelta T cell responses in bacterial lung infections and lethal pneumonia. Nat. Med. 24, 417-426. 10.1038/nm.4501.

Bautista, D.M., Jordt, S.E., Nikai, T., Tsuruda, P.R., Read, A.J., Poblete, J., Yamoah, E.N., Basbaum, A.I., and Julius, D. (2006). TRPA1 mediates the inflammatory actions of environmental irritants and proalgesic agents. Cell 124, 1269-1282. 10.1016/j.cell.2006.02.023. Brookes, S.J., Spencer, N.J., Costa, M., and Zagorodnyuk, V.P. (2013). Extrinsic primary afferent signalling in the gut. Nat. Rev. Gastroenterol. Hepatol. 10, 286-296. 10.1038/nrgastro.2013.29.

Brouns, I., De Proost, I., Pintelon, I., Timmermans, J.P., and Adriaensen, D. (2006). Sensory receptors in the airways: neurochemical coding of smooth muscle-associated airway receptors 
and pulmonary neuroepithelial body innervation. Auton. Neurosci. 126-127, 307-319. 10.1016/j.autneu.2006.02.006.

Brouns, I., Oztay, F., Pintelon, I., De Proost, I., Lembrechts, R., Timmermans, J.P., and Adriaensen, D. (2009). Neurochemical pattern of the complex innervation of neuroepithelial bodies in mouse lungs. Histochem. Cell Biol. 131, 55-74. 10.1007/s00418-008-0495-7.

Brouns, I., Pintelon, I., Timmermans, J.P., and Adriaensen, D. (2012). Novel insights in the neurochemistry and function of pulmonary sensory receptors. Adv. Anat. Embryol. Cell Biol. 211, 1-115, vii.

Cameron, O.G. (2002). Visceral sensory neuroscience : interoception (Oxford University Press). Canning, B.J., Mori, N., and Mazzone, S.B. (2006). Vagal afferent nerves regulating the cough reflex. Respir. Physiol. Neurobiol. 152, 223-242. 10.1016/j.resp.2006.03.001.

Cervero, F., R. D. Foreman (1990). Sensory innervation of the viscera. In Central regulation of autonomic functions, K.M.S. A. D. Loewy, ed. (Oxford University Press), pp. 104-125.

Chang, A.J., Ortega, F.E., Riegler, J., Madison, D.V., and Krasnow, M.A. (2015a). Oxygen regulation of breathing through an olfactory receptor activated by lactate. Nature 527, 240-244. 10.1038/nature15721.

Chang, R.B., Strochlic, D.E., Williams, E.K., Umans, B.D., and Liberles, S.D. (2015b). Vagal sensory neuron subtypes that differentially control breathing. Cell 161, 622-633. 10.1016/j.cell.2015.03.022.

Chistiakov, D.A., Orekhov, A.N., and Bobryshev, Y.V. (2017). Effects of shear stress on endothelial cells: go with the flow. Acta Physiol. (Oxf.) 219, 382-408. 10.1111/apha.12725.

Clark, F.J., and von Euler, C. (1972). On the regulation of depth and rate of breathing. J. Physiol. 222, 267-295. 10.1113/jphysiol.1972.sp009797. 
Cohen, J.A., Wu, J., and Kaplan, D.H. (2020). Neuronal regulation of cutaneous immunity. J. Immunol. 204, 264-270. 10.4049/jimmunol.1901109.

Coleridge, H.M., and Coleridge, J.C.G. (2011). Reflexes evoked from tracheobronchial tree and lungs. In Comprehensive Physiology, (John Wiley \& Sons, Inc. Hoboken, NJ), pp. 395-429. 10.1002/cphy.cp030212.

Craig, A.D. (2002). How do you feel? Interoception: the sense of the physiological condition of the body. Nat. Rev. Neurosci. 3, 655-666. 10.1038/nrn894.

Danielian, P.S., Muccino, D., Rowitch, D.H., Michael, S.K., and McMahon, A.P. (1998).

Modification of gene activity in mouse embryos in utero by a tamoxifen-inducible form of Cre recombinase. Curr. Biol. 8, 1323-1326. 10.1016/s0960-9822(07)00562-3.

Dauger, S., Pattyn, A., Lofaso, F., Gaultier, C., Goridis, C., Gallego, J., and Brunet, J.F. (2003).

Phox $2 \mathrm{~b}$ controls the development of peripheral chemoreceptors and afferent visceral pathways. Development 130, 6635-6642. 10.1242/dev.00866.

Dinh, Q.T., Mingomataj, E., Quarcoo, D., Groneberg, D.A., Witt, C., Klapp, B.F., Braun, A., and Fischer, A. (2005). Allergic airway inflammation induces tachykinin peptides expression in vagal sensory neurons innervating mouse airways. Clin. Exp. Allergy 35, 820-825.

10.1111/j.1365-2222.2005.02264.x.

Dockray, G.J. (2013). Enteroendocrine cell signalling via the vagus nerve. Curr. Opin.

Pharmacol. 13, 954-958. 10.1016/j.coph.2013.09.007.

Driessen, A.K., McGovern, A.E., Narula, M., Yang, S.K., Keller, J.A., Farrell, M.J., and Mazzone, S.B. (2017). Central mechanisms of airway sensation and cough hypersensitivity. Pulm. Pharmacol. Ther. 47, 9-15. 10.1016/j.pupt.2017.01.010. 
Evans, R.C., Zhu, M., and Khaliq, Z.M. (2017). Dopamine inhibition differentially controls excitability of substantia nigra dopamine neuron subpopulations through T-type calcium channels. J. Neurosci. 37, 3704-3720. 10.1523/jneurosci.0117-17.2017.

Fajardo, O., Meseguer, V., Belmonte, C., and Viana, F. (2008). TRPA1 channels mediate cold temperature sensing in mammalian vagal sensory neurons: pharmacological and genetic evidence. J. Neurosci. 28, 7863-7875. 10.1523/jneurosci.1696-08.2008.

Fox, E.A., Phillips, R.J., Martinson, F.A., Baronowsky, E.A., and Powley, T.L. (2001). C-Kit mutant mice have a selective loss of vagal intramuscular mechanoreceptors in the forestomach. Anat. Embryol. (Berl.) 204, 11-26. 10.1007/s004290100184.

Garfield, A.S., Li, C., Madara, J.C., Shah, B.P., Webber, E., Steger, J.S., Campbell, J.N., Gavrilova, O., Lee, C.E., Olson, D.P., et al. (2015). A neural basis for melanocortin-4 receptorregulated appetite. Nat. Neurosci. 18, 863-871. 10.1038/nn.4011.

Griffiths, C.D., Bilawchuk, L.M., McDonough, J.E., Jamieson, K.C., Elawar, F., Cen, Y., Duan, W., Lin, C., Song, H., Casanova, J.L., et al. (2020). IGF1R is an entry receptor for respiratory syncytial virus. Nature 583, 615-619. 10.1038/s41586-020-2369-7.

Grundy, L., Erickson, A., and Brierley, S.M. (2019). Visceral pain. Annu. Rev. Physiol. 81, 261284. 10.1146/annurev-physiol-020518-114525.

Han, L., Limjunyawong, N., Ru, F., Li, Z., Hall, O.J., Steele, H., Zhu, Y., Wilson, J., Mitzner, W., Kollarik, M., et al. (2018). Mrgprs on vagal sensory neurons contribute to bronchoconstriction and airway hyper-responsiveness. Nat. Neurosci. 21, 324-328. 10.1038/s41593-018-0074-8. 
Hayashi, F., Smith, K.D., Ozinsky, A., Hawn, T.R., Yi, E.C., Goodlett, D.R., Eng, J.K., Akira, S., Underhill, D.M., and Aderem, A. (2001). The innate immune response to bacterial flagellin is mediated by Toll-like receptor 5. Nature 410, 1099-1103. 10.1038/35074106.

Hockley, J.R.F., Taylor, T.S., Callejo, G., Wilbrey, A.L., Gutteridge, A., Bach, K., Winchester, W.J., Bulmer, D.C., McMurray, G., and Smith, E.S.J. (2019). Single-cell RNAseq reveals seven classes of colonic sensory neuron. Gut 68, 633-644. 10.1136/gutjnl-2017-315631.

Horn, C.C. (2014). The medical implications of gastrointestinal vagal afferent pathways in nausea and vomiting. Curr. Pharm. Des. 20, 2703-2712. 10.2174/13816128113199990568. Hsu, C.C., Ruan, T., Lee, L.Y., and Lin, Y.S. (2019). Stimulatory effect of 5-Hydroxytryptamine (5-HT) on rat capsaicin-sensitive lung vagal sensory neurons via activation of 5-HT(3) receptors. Front. Physiol. 10, 642. 10.3389/fphys.2019.00642.

Hu, J., Zhong, C., Ding, C., Chi, Q., Walz, A., Mombaerts, P., Matsunami, H., and Luo, M. (2007). Detection of near-atmospheric concentrations of CO2 by an olfactory subsystem in the mouse. Science 317, 953-957. 10.1126/science.1144233.

Jain, A., Hakim, S., and Woolf, C.J. (2020). Unraveling the plastic peripheral neuroimmune interactome. J. Immunol. 204, 257-263. 10.4049/jimmunol.1900818.

Jensen, K.J., Alpini, G., and Glaser, S. (2013). Hepatic nervous system and neurobiology of the liver. Compr. Physiol. 3, 655-665. 10.1002/cphy.c120018.

Kawayama, T., Okamoto, M., Imaoka, H., Kato, S., Young, H.A., and Hoshino, T. (2012).

Interleukin-18 in pulmonary inflammatory diseases. J. Interferon Cytokine Res. 32, 443-449. 10.1089/jir.2012.0029.

Kharchenko, P.V., Silberstein, L., and Scadden, D.T. (2014). Bayesian approach to single-cell differential expression analysis. Nat. Methods 11, 740-742. 10.1038/nmeth.2967. 
Kim, E.J., Zhang, Z., Huang, L., Ito-Cole, T., Jacobs, M.W., Juavinett, A.L., Senturk, G., Hu, M., Ku, M., Ecker, J.R., and Callaway, E.M. (2020). Extraction of distinct neuronal cell types from within a genetically continuous population. Neuron 107, 274-282.e276.

10.1016/j.neuron.2020.04.018.

Kubo, M. (2017). Innate and adaptive type 2 immunity in lung allergic inflammation. Immunol. Rev. 278, 162-172. 10.1111/imr.12557.

Kumada, M., Terui, N., and Kuwaki, T. (1990). Arterial baroreceptor reflex: its central and peripheral neural mechanisms. Prog. Neurobiol. 35, 331-361. 10.1016/0301-0082(90)90036-g. Kumar, P., and Prabhakar, N.R. (2012). Peripheral chemoreceptors: function and plasticity of the carotid body. Compr. Physiol. 2, 141-219. 10.1002/cphy.c100069.

Kupari, J., Haring, M., Agirre, E., Castelo-Branco, G., and Ernfors, P. (2019). An atlas of vagal sensory neurons and their molecular specialization. Cell Rep. 27, 2508-2523 e2504. 10.1016/j.celrep.2019.04.096.

Kwong, K., and Lee, L.Y. (2002). PGE(2) sensitizes cultured pulmonary vagal sensory neurons to chemical and electrical stimuli. J. Appl. Physiol. (1985) 93, 1419-1428.

10.1152/japplphysiol.00382.2002.

Larshell, O., and Dow, R.S. (1933). The innervation of the human lung. Am. J. Anat. 52, 125146. 10.1002/aja.1000520104.

Lauweryns, J.M., Cokelaere, M., Theunynck, P., and Deleersnyder, M. (1974). Neuroepithelial bodies in mammalian respiratory mucosa: light optical, histochemical an ultrastructural studies. Chest 65, Suppl:22S-29S. 10.1378/chest.65.4_supplement.22s. 
Lawson, S.N., and Waddell, P.J. (1991). Soma neurofilament immunoreactivity is related to cell size and fibre conduction velocity in rat primary sensory neurons. J. Physiol. 435, 41-63. 10.1113/jphysiol.1991.sp018497.

Lee, L.Y., Gu, Q., Xu, F., and Hong, J.L. (2013). Acid-sensing by airway afferent nerves. Pulm. Pharmacol. Ther. 26, 491-497. 10.1016/j.pupt.2013.03.010.

Lee, L.Y., Lin, R.L., Khosravi, M., and Xu, F. (2018). Reflex bronchoconstriction evoked by inhaled nicotine aerosol in guinea pigs: role of the nicotinic acetylcholine receptor. J. Appl. Physiol. (1985) 125, 117-123. 10.1152/japplphysiol.01039.2017.

Lee, L.Y., and Pisarri, T.E. (2001). Afferent properties and reflex functions of bronchopulmonary C-fibers. Respir. Physiol. 125, 47-65. 10.1016/s0034-5687(00)00204-8.

Lee, L.Y., and Yu, J. (2014). Sensory nerves in lung and airways. Compr. Physiol. 4, 287-324. 10.1002/cphy.c130020.

Li, C.L., Li, K.C., Wu, D., Chen, Y., Luo, H., Zhao, J.R., Wang, S.S., Sun, M.M., Lu, Y.J., Zhong, Y.Q., et al. (2016). Somatosensory neuron types identified by high-coverage single-cell RNA-sequencing and functional heterogeneity. Cell Res. 26, 83-102. 10.1038/cr.2015.149.

Lin, R.L., Gu, Q., Lin, Y.S., and Lee, L.Y. (2005). Stimulatory effect of CO2 on vagal bronchopulmonary C-fiber afferents during airway inflammation. J. Appl. Physiol. (1985) 99, 1704-1711. 10.1152/japplphysiol.00532.2005.

Lin, R.L., Hayes, D., Jr., and Lee, L.Y. (2009). Bronchoconstriction induced by hyperventilation with humidified hot air: role of TRPV1-expressing airway afferents. J. Appl. Physiol. (1985) 106, 1917-1924. 10.1152/japplphysiol.00065.2009.

Longhurst, J.C. (1984). Cardiac receptors: their function in health and disease. Prog. Cardiovasc. Dis. 27, 201-222. 10.1016/0033-0620(84)90005-7. 
Ludwig, M.G., Vanek, M., Guerini, D., Gasser, J.A., Jones, C.E., Junker, U., Hofstetter, H., Wolf, R.M., and Seuwen, K. (2003). Proton-sensing G-protein-coupled receptors. Nature 425, 93-98. 10.1038/nature01905.

Ma, A., Bravo, M., and Kappagoda, C.T. (2003). Responses of bronchial C-fiber afferents of the rabbit to changes in lung compliance. Respir. Physiol. Neurobiol. 138, 155-163. 10.1016/s15699048(03)00189-7.

Matsumoto, S., Okamura, H., Suzuki, K., Sugai, N., and Shimizu, T. (1996). Inhibitory mechanism of $\mathrm{CO} 2$ inhalation on slowly adapting pulmonary stretch receptors in the anesthetized rabbit. J. Pharmacol. Exp. Ther. 279, 402-409.

Mazzone, S.B., Tian, L., Moe, A.A.K., Trewella, M.W., Ritchie, M.E., and McGovern, A.E. (2020). Transcriptional Profiling of Individual Airway Projecting Vagal Sensory Neurons. Mol. Neurobiol. 57, 949-963. 10.1007/s12035-019-01782-8.

Mazzone, S.B., and Undem, B.J. (2016). Vagal afferent innervation of the airways in health and disease. Physiol. Rev. 96, 975-1024. 10.1152/physrev.00039.2015.

McGonagle, D., Sharif, K., O'Regan, A., and Bridgewood, C. (2020). The role of cytokines including Interleukin-6 in COVID-19 induced pneumonia and macrophage activation syndromelike disease. Autoimmun Rev 19, 102537. 10.1016/j.autrev.2020.102537.

Moreira, T.S., Takakura, A.C., Colombari, E., and Guyenet, P.G. (2007). Activation of 5hydroxytryptamine type 3 receptor-expressing C-fiber vagal afferents inhibits retrotrapezoid nucleus chemoreceptors in rats. J. Neurophysiol. 98, 3627-3637. 10.1152/jn.00675.2007. Mühlebach, M.D., Mateo, M., Sinn, P.L., Prüfer, S., Uhlig, K.M., Leonard, V.H.J., Navaratnarajah, C.K., Frenzke, M., Wong, X.X., Sawatsky, B., et al. (2011). Adherens junction 
protein nectin-4 is the epithelial receptor for measles virus. Nature 480, 530-533.

10.1038/nature10639.

Nassenstein, C., Kwong, K., Taylor-Clark, T., Kollarik, M., Macglashan, D.M., Braun, A., and Undem, B.J. (2008). Expression and function of the ion channel TRPA1 in vagal afferent nerves innervating mouse lungs. J. Physiol. 586, 1595-1604. 10.1113/jphysiol.2007.148379.

Nassenstein, C., Taylor-Clark, T.E., Myers, A.C., Ru, F., Nandigama, R., Bettner, W., and Undem, B.J. (2010). Phenotypic distinctions between neural crest and placodal derived vagal Cfibres in mouse lungs. J. Physiol. 588, 4769-4783. 10.1113/jphysiol.2010.195339.

Nishino, T., Ide, T., Sudo, T., and Sato, J. (2000). Inhaled furosemide greatly alleviates the sensation of experimentally induced dyspnea. Am. J. Respir. Crit. Care Med. 161, 1963-1967. 10.1164/ajrccm.161.6.9910009.

Nonomura, K., Woo, S.H., Chang, R.B., Gillich, A., Qiu, Z., Francisco, A.G., Ranade, S.S., Liberles, S.D., and Patapoutian, A. (2017). Piezo2 senses airway stretch and mediates lung inflation-induced apnoea. Nature 541, 176-181. 10.1038/nature20793.

Pan, B., Geleoc, G.S., Asai, Y., Horwitz, G.C., Kurima, K., Ishikawa, K., Kawashima, Y., Griffith, A.J., and Holt, J.R. (2013). TMC1 and TMC2 are components of the mechanotransduction channel in hair cells of the mammalian inner ear. Neuron 79, 504-515. 10.1016/j.neuron.2013.06.019.

Pirooz, S.D., He, S., Zhang, T., Zhang, X., Zhao, Z., Oh, S., O'Connell, D., Khalilzadeh, P., Amini-Bavil-Olyaee, S., Farzan, M., and Liang, C. (2014). UVRAG is required for virus entry through combinatorial interaction with the class C-Vps complex and SNAREs. Proc. Natl. Acad. Sci. U. S. A. 111, 2716-2721. 10.1073/pnas.1320629111. 
Prescott, S.L., Umans, B.D., Williams, E.K., Brust, R.D., and Liberles, S.D. (2020). An airway protection program revealed by sweeping genetic control of vagal afferents. Cell $181,574-$ 589.e514. 10.1016/j.cell.2020.03.004.

Quadt, L., Critchley, H.D., and Garfinkel, S.N. (2018). The neurobiology of interoception in health and disease. Ann. N. Y. Acad. Sci. 1428, 112-128. 10.1111/nyas.13915.

Raybould, H.E. (2010). Gut chemosensing: interactions between gut endocrine cells and visceral afferents. Auton. Neurosci. 153, 41-46. 10.1016/j.autneu.2009.07.007.

Ruan, T., Gu, Q., Kou, Y.R., and Lee, L.Y. (2005). Hyperthermia increases sensitivity of pulmonary C-fibre afferents in rats. J. Physiol. 565, 295-308. 10.1113/jphysiol.2005.084319. Schelegle, E.S. (2003). Functional morphology and physiology of slowly adapting pulmonary stretch receptors. Anat. Rec. A Discov. Mol. Cell. Evol. Biol. 270, 11-16. 10.1002/ar.a.10004. Schelegle, E.S., and Green, J.F. (2001). An overview of the anatomy and physiology of slowly adapting pulmonary stretch receptors. Respir. Physiol. 125, 17-31. 10.1016/s0034$5687(00) 00202-4$

Scott, M.M., Williams, K.W., Rossi, J., Lee, C.E., and Elmquist, J.K. (2011). Leptin receptor expression in hindbrain Glp-1 neurons regulates food intake and energy balance in mice. J. Clin. Invest. 121, 2413-2421. 10.1172/jci43703.

Sharma, N., Flaherty, K., Lezgiyeva, K., Wagner, D.E., Klein, A.M., and Ginty, D.D. (2020).

The emergence of transcriptional identity in somatosensory neurons. Nature 577, 392-398. 10.1038/s41586-019-1900-1.

Sherrington, C.S. (1906). The integrative action of the nervous system (C. Scribner's sons). 
Song, H., Yao, E., Lin, C., Gacayan, R., Chen, M.H., and Chuang, P.T. (2012). Functional characterization of pulmonary neuroendocrine cells in lung development, injury, and tumorigenesis. Proc. Natl. Acad. Sci. U. S. A. 109, 17531-17536. 10.1073/pnas.1207238109. Stanley, G., Gokce, O., Malenka, R.C., Südhof, T.C., and Quake, S.R. (2020). Continuous and Discrete Neuron Types of the Adult Murine Striatum. Neuron 105, 688-699.e688. 10.1016/j.neuron.2019.11.004.

Suminaite, D., Lyons, D.A., and Livesey, M.R. (2019). Myelinated axon physiology and regulation of neural circuit function. Glia 67, 2050-2062. 10.1002/glia.23665.

Tabula Muris, C. (2018). Single-cell transcriptomics of 20 mouse organs creates a Tabula Muris. Nature 562, 367-372. 10.1038/s41586-018-0590-4.

Tabula Muris, C. (2020). A single-cell transcriptomic atlas characterizes ageing tissues in the mouse. Nature 583, 590-595. 10.1038/s41586-020-2496-1.

Taguchi, O., Kikuchi, Y., Hida, W., Iwase, N., Satoh, M., Chonan, T., and Takishima, T. (1991). Effects of bronchoconstriction and external resistive loading on the sensation of dyspnea. J. Appl. Physiol. (1985) 71, 2183-2190. 10.1152/jappl.1991.71.6.2183.

Talbot, S., Abdulnour, R.E., Burkett, P.R., Lee, S., Cronin, S.J., Pascal, M.A., Laedermann, C., Foster, S.L., Tran, J.V., Lai, N., et al. (2015). Silencing nociceptor neurons reduces allergic airway inflammation. Neuron 87, 341-354. 10.1016/j.neuron.2015.06.007.

Tasic, B., Menon, V., Nguyen, T.N., Kim, T.K., Jarsky, T., Yao, Z., Levi, B., Gray, L.T., Sorensen, S.A., Dolbeare, T., et al. (2016). Adult mouse cortical cell taxonomy revealed by single cell transcriptomics. Nat. Neurosci. 19, 335-346. 10.1038/nn.4216.

Taylor-Clark, T.E., and Undem, B.J. (2010). Ozone activates airway nerves via the selective stimulation of TRPA1 ion channels. J. Physiol. 588, 423-433. 10.1113/jphysiol.2009.183301. 
Tetzlaff, K., Leplow, B., ten Thoren, C., and Dahme, B. (1999). Perception of dyspnea during histamine- and methacholine-induced bronchoconstriction. Respiration 66, 427-433.

$10.1159 / 000029426$.

Trapp, S., Aller, M.I., Wisden, W., and Gourine, A.V. (2008). A role for TASK-1 (KCNK3)

channels in the chemosensory control of breathing. J. Neurosci. $28,8844-8850$.

10.1523/jneurosci.1810-08.2008.

Travaglini, K.J., Nabhan, A.N., Penland, L., Sinha, R., Gillich, A., Sit, R.V., Chang, S., Conley, S.D., Mori, Y., Seita, J., et al. (2020). A molecular cell atlas of the human lung from single-cell RNA sequencing. Nature 587, 619-625. 10.1038/s41586-020-2922-4.

Ullmann, E. (1970). The two original papers by Hering and Breuer submitted by Hering to the K.K. Akademie Der Wissenschaften Zu Wien in 1868. In Ciba Foundation Symposium -

Breathing: Hering-Breuer Centenary Symposium, pp. 357-394. 10.1002/9780470715352.ch20.

Umans, B.D., and Liberles, S.D. (2018). Neural sensing of organ volume. Trends Neurosci. 41, 911-924. 10.1016/j.tins.2018.07.008.

Verzele, N.A.J., Chua, B.Y., Law, C.W., Zhang, A., Ritchie, M.E., Wightman, O., Edwards, I.N., Hulme, K.D., Bloxham, C.J., Bielefeldt-Ohmann, H., et al. (2021). The impact of influenza pulmonary infection and inflammation on vagal bronchopulmonary sensory neurons. FASEB J. 35, e21320. 10.1096/fj.202001509R.

Waise, T.M.Z., Dranse, H.J., and Lam, T.K.T. (2018). The metabolic role of vagal afferent innervation. Nat. Rev. Gastroenterol. Hepatol. 15, 625-636. 10.1038/s41575-018-0062-1.

Widdicombe, J. (2006). Reflexes from the lungs and airways: historical perspective. J. Appl. Physiol. (1985) 101, 628-634. 10.1152/japplphysiol.00155.2006. 
Williams, E.K., Chang, R.B., Strochlic, D.E., Umans, B.D., Lowell, B.B., and Liberles, S.D. (2016). Sensory neurons that detect stretch and nutrients in the digestive system. Cell 166, 209221. 10.1016/j.cell.2016.05.011.

Xiong, W., Grillet, N., Elledge, H.M., Wagner, T.F., Zhao, B., Johnson, K.R., Kazmierczak, P., and Muller, U. (2012). TMHS is an integral component of the mechanotransduction machinery of cochlear hair cells. Cell 151, 1283-1295. 10.1016/j.cell.2012.10.041.

Yu, J. (2005). Airway mechanosensors. Respir. Physiol. Neurobiol. 148, 217-243. 10.1016/j.resp.2004.12.007.

Zhang, G., Lin, R.L., Wiggers, M., Snow, D.M., and Lee, L.Y. (2008). Altered expression of TRPV1 and sensitivity to capsaicin in pulmonary myelinated afferents following chronic airway inflammation in the rat. J. Physiol. 586, 5771-5786. 10.1113/jphysiol.2008.161042.

Zhang, J.W., Walker, J.F., Guardiola, J., and Yu, J. (2006). Pulmonary sensory and reflex responses in the mouse. J. Appl. Physiol. (1985) 101, 986-992.

10.1152/japplphysiol.00161.2006.

Zhang, S., Edwards, T.N., Chaudhri, V.K., Wu, J., Cohen, J.A., Hirai, T., Rittenhouse, N., Schmitz, E.G., Zhou, P.Y., McNeil, B.D., et al. (2021). Nonpeptidergic neurons suppress mast cells via glutamate to maintain skin homeostasis. Cell 184, 2151-2166.e2116.

10.1016/j.cell.2021.03.002.

Zhao, B., Wu, Z., Grillet, N., Yan, L., Xiong, W., Harkins-Perry, S., and Muller, U. (2014).

TMIE is an essential component of the mechanotransduction machinery of cochlear hair cells.

Neuron 84, 954-967. 10.1016/j.neuron.2014.10.041. 
bioRxiv preprint doi: https://doi.org/10.1101/2021.11.10.468116; this version posted November 13,2021 . The copyright holder for this preprint (which was not certified by peer review) is the author/funder. All rights reserved. No reuse allowed without permission.

Liu et al., p. 46

Zhao, Y., Yang, J., Shi, J., Gong, Y.-N., Lu, Q., Xu, H., Liu, L., and Shao, F. (2011). The

NLRC4 inflammasome receptors for bacterial flagellin and type III secretion apparatus. Nature

477, 596-600. 10.1038/nature10510. 


\section{FIGURE LEGENDS}

\section{Figure 1. Labeling, transcriptomic profiling, and identification of 10 molecular subtypes of vagal PSNs}

(A) Strategy for scRNA-seq of vagal PSNs. PSNs were labeled by intratracheal instillation of a fluorescent WGA conjugate (green). Vagal ganglia (J, jugular ganglion; N, nodose ganglion) were dissociated, and WGA-labeled neurons were individually picked and placed in separate tubes. scRNA-seq was carried out in each tube using the Smart-seq2 (SS2) method.

(B, C) Retrograde labeling with two fluorescent WGA conjugates (WGA-488, green; WGA-555, red) as indicated of vagal sensory neurons from the lung ( $\mathrm{n}=3$ mice) or from the lung and the stomach ( $\mathrm{n}=2$ mice). Images are maximum projections of the entire ganglia after BABB clearance, with quantifications at right. n, total labeled neurons scored (both fluorescent labels).

(D) Computational cell clustering pipeline for subtype identification based on similarity of scRNA-seq expression profiles. In each round of iterative clustering (hierarchical steps, left to right), cells were clustered into two groups based on highly variable genes, and only neurons that stably clustered together were promoted to the next round (each intermediate cluster numbered as indicated, e.g., I.1). Iteration ended when no statistically significant separation was generated, or the number of cells in one group was $<5$. Cells in intermediate clusters are shown in black or white (gray cells did not stably cluster), and cells in the final clusters are color coded and their subtype designation given at right along with predicted fiber type and developmental origin from results in panels F-H. Number of neurons in each cluster are indicated in parentheses. All plots are tSNE projections except two marked with PC, which show only the first two principal components of PCA because no more than two PCs were statistically significant. 
(E) Heatmap showing the expression pattern of genes that are differentially expressed between PSN2 and PSN3 neurons across PSN2, H and 3. Many ion channels and GPCRs (indicated) exhibit a gradient of expression in PSN-H neurons, suggesting they are functional intermediates. (F-H) Cell diameters (F) and expression levels of Nefh (G, correlates with myelination states) and Phox $2 b$ (H, developmental lineage marker) of neurons of each PSN subtype. Dots represent values for individual neurons, and hinges correspond to $25^{\text {th }}$ and $75^{\text {th }}$ percentiles. 


\section{Figure 2. Markers, abundance, and anatomical features of PSN subtypes.}

(A) Dot plot showing expression levels of PSN subtype marker genes. Numbers at bottom are mean $\log _{2}(\mathrm{TPM}+1)$ values of the subtypes with the highest average expression levels of the indicated genes, with a threshold set at TPM $>=1$ (TPM, transcript per million). This format is used for expression dot plots in all figures, unless noted otherwise. Genes in red were used for validation analysis by smFISH and immunostaining in (B) and Figure S2C. Group I and II correspond to the two clusters generated in the first round of iterative clustering shown in Figure $1 \mathrm{D}$.

(B) smFISH (RNAscope) or immunostaining of the indicated PSN subtype markers on mouse vagal ganglion sections with WGA retrograde labeling from the lung. Close-ups of boxed areas are shown in insets with channels split, and the outlined neurons are examples of indicated PSN subtypes. Scale bars: $20 \mu \mathrm{m}$ and $10 \mu \mathrm{m}$ (insets). Examples of PSN2, H, 3 and 9 are shown in Figure S2C and D.

(C) Estimated number of PSN neurons of the indicated subtypes in each vagal ganglion based on quantification of smFISH (in vivo, solid bars) and proportions of all sequenced neurons (scRNAseq, open bars). Values were scaled to total 400 PSNs per ganglion. PSN2, H and 3 are plotted together because no unique markers identify PSN-H neurons, but from the scRNAseq analysis there are comparable numbers of all three types. Quantification sample sizes are given in Figure S2E.

(D) Estimated proportions of individual PSN subtypes. All subtypes except PSN2, H, and 3 are estimated based on smFISH results. PSN2, 3 and $\mathrm{H}$ are estimated based on scRNA-seq results (divided by dashed lines). Sum of the estimated proportions totals $105 \%$, indicating the 10 subtypes account for all PSNs labeled by our general labeling method. 
(E-H) Group I neurons labeled by injecting AAV-CAG-FLEX-tdTomato into vagal ganglia of $\operatorname{Trpv} 1^{\mathrm{Cre} /+}$ mice. E and F, maximum projections of a segment of conducting airways (E) and a region mainly occupied by alveoli (F). Sections were immunostained for substance P (SP, selectively expressed in PSN1 among Trpv1-expressing PSNs, Figure S2B), tdTomato and smooth muscle actin (SMA, surrounds conducting airways and major blood vessels and is absent in alveolar regions). Close ups of boxed regions are shown in E' and F'. E' shows both $\mathrm{SP}^{+}$ (single arrowheads, PSN1) and $\mathrm{SP}^{-}$(triple arrowhead, likely PSN3 or H) labeled fibers. F' shows a SP- labeled fiber (double arrowhead, likely PSN2 or H) innervating alveoli. Note that virtually all alveolar tdTomato ${ }^{+}$fibers were $\mathrm{SP}^{-}$. G, a thin optical section of alveoli immunostained with tdTomato, Rage (alveolar type I cell marker), and Pecam (capillary marker), showing an alveolar Group I neuron terminal, presumably a PSN2 or H neuron, contacting both alveolar cell types. H, maximum projection of alveoli from the same lung section as G. Note that most Group I alveolar fibers run along the junctions between alveoli, sending rare branches onto the alveolar surface (Figure S3F). bv, blood vessel; ca, conducting airway. Scale bars: $100 \mu \mathrm{m}$ (E and F), $10 \mu \mathrm{m}$ (E'), $20 \mu \mathrm{m}\left(\mathrm{F}^{\prime}\right), 5 \mu \mathrm{m}(\mathrm{G}), 10 \mu \mathrm{m}(\mathrm{H})$.

(I-K) PSN6 terminals labeled by injecting AAV-Syn-DIO-hM3Dq-mCherry into the vagal ganglia of $M c 4 r^{2 \mathrm{~A}-\mathrm{Cre} /+}$ mice. I, maximum projection of a bronchial branch point with two labeled terminals (arrowheads). J, top-down view of a labeled terminal showing multi-branched "leaf" shape morphology. K, side view of a labeled terminal intercalating with airway smooth muscle fibers $\left(\mathrm{SMA}^{+}\right.$). al, airway lumen; e, epithelium (outlined). Scale bars: $100 \mu \mathrm{m}$ (I), $20 \mu \mathrm{m}$ (J and K).

(L-N) PSN7 terminals labeled by injecting AAV-Syn-DIO-hM3Dq-mCherry into the vagal ganglia of Calb1 ${ }^{2 \mathrm{~A}-\mathrm{dgCre} /+}$ mice, followed by TMP induction. L, maximum projection of 
conducting airways with three NEBs (labeled by CGRP immunostaining) all innervated by labeled terminals (arrowheads). M, top-down view of a labeled terminal showing "waffle" shape morphology, N, side view of a labeled terminal covering the apical side of the NEB. al, airway lumen; e, epithelium (outlined). Scale bars: $100 \mu \mathrm{m}(\mathrm{L}), 10 \mu \mathrm{m}(\mathrm{M}), 20 \mu \mathrm{m}(\mathrm{N})$.

(O) Diagrams of 6 PSN subtypes showing their terminal structures, locations, and contacted lung cells. PSN1, 2, H and 3 all form free nerve endings. PSN1 and 3 both branch along conducting airways beneath airway smooth muscle. PSN2 mainly branches along the alveolar junctions and closely contacts alveolar type I and capillary cells. PSN6 terminals intercalate with airway smooth muscle, preferentially at bronchial branch points. PSN7 also terminates at branch points but on the apical side of NEBs. Boxes show top-down views of PSN6 and PSN7 termini. ASM, airway smooth muscle; PNEC, pulmonary neuroendocrine cell; AT1, alveolar type I cell; Cap, capillary cell.

(P) Distribution of PSN subtypes throughout a lung lobe. PSN1 and PSN3 both terminate on conducting airways (light purple), whereas PSN2 terminates predominately in the alveolar region (light blue) (whether PSN3 also innervates alveoli is undetermined). PSN-H likely exhibits a similar distribution as PSN2 or PSN3 or a combination of both. Group I neurons also terminate on pulmonary blood vessels (not shown). PSN6 (orange dots) and PSN7 (red dots) both terminate at bronchial branch points but with different proximal and distal distributions as shown. However, the terminal locations of PSN6 and 7 neurons are not stereotyped between different lobes or animals, and the schematic only illustrates general features of their terminal distributions. 
Figure 3. Diversity of PSNs in physiological sensing, pathogen detection and neuronal signaling

(A) Dot plots showing expression in PSN subtypes of genes encoding receptors that detect the diverse physiological stimuli indicated. Expression levels of nicotinic acetylcholine receptor subunits (*) are the summed values of all isoforms (see Figure S4A for individual isoforms).

(B) Expression of pathogen pattern recognition receptors for bacterial cellular components. LPS, lipopolysaccharide; PGN, peptidoglycan.

(C) Expression of genes encoding neuropeptides and synthesis and release components for the indicated neurotransmitters. Glu, glutamate; ACh, acetylcholine, DP, dopamine, NE, norepinephrine, NO, nitric oxide.

(D) Summary of predicted physiological sensing and neuronal signaling for the four PSN subtypes indicated. PSNs are pseudounipolar neurons, with one branch projecting to the lung (left) and the other to the brainstem (right). Stimuli indicated here are presumably detected by the peripheral terminals in the lung, and neurotransmitters and neuropeptides are likely released from the central terminals in the brainstem. Neuropeptides like CGRP and SP (asterisks) are also known to be released in the lung. Remaining PSNs are shown in Figure S4B. CS, cigarette smoke; Mech, mechanical stimuli. 


\section{Figure 4. PSNs sensing inflammation and interacting with lung immune cells}

(A) Expression of receptors in PSNs detecting the indicated non-cytokine inflammatory mediators. LPA, lysophosphatidic acid; S1P, sphingosine-1-phosphate; PG, prostaglandins; LT, leukotrienes.

(B) Expression of selected cytokine ligands and their cognate receptors in lung immune cell types (left; dashed line divides innate and adaptive immune cell types) and PSN subtypes (right). Red, blue, and green lines and arrows denote proposed reciprocal signaling pathways between lung immune cells and PSNs: Basophils can signal to PSN1 via IL4, and PSN1 can signal to monocytes and dendritic cells via CCL2, as well as back to basophils via IL18. mDC1, myeloid dendritic type 1 cells; mDC2, myeloid dendritic type 2 cells; Ccr7+ DC, Ccr7+ dendritic cells; NC Mono, non-classical monocytes; inter Mono, intermediate monocytes; C Mono, classical monocytes; B, B cells; CD4+ T, CD4+ T cells; CD8+ T, CD8+ T cells; NK, natural killer cells; Neu, neutrophils; Bas, basophils; InterM $\phi$, interstitial macrophages; AlvM $\phi$, alveolar macrophages.

(C) Summary of inflammatory mediators detected (blue) and immune cell-signaling cytokines secreted (magenta molecules) by four PSN subtypes. Light blue and light magenta indicate that the ligand or receptor is expressed at a lower level compared to other subtypes $(50-75 \%$ of the mean level in the subtype with highest expression). Other PSNs are shown in Figure S6. 
Figure 5. Ablation of PSN6 neurons increases tidal volume and inspiratory time.

(A) Experimental scheme for assessing functions of PSN subtypes in breathing regulation. AAVDIO-DTR-GFP was bilaterally injected into the vagal ganglia of subtype-specific Cre mice.

Three weeks (20-24 days) later, DT was injected intraperitoneally to ablate the targeted PSN subtypes. Whole-body plethysmography (WBP) recordings to acquire breathing parameters were performed 1 day before (pre-ablation) and 3 days after DT injection (post-ablation). WGA retrograde labeling was performed 5 days prior to DT injection to label PSNs for later assessment of ablation efficiency.

(B) Kernel density plots showing tidal volume (TV) distribution for calm breaths of a control and a PSN6-DTR mouse pre- and post-DT injection. Note shift to higher tidal volumes following PSN6 ablation.

(C) Tidal volume (TV) changes after PSN6 ablation. Each bar represents the mean ratio of TV distribution mode post- to pre-DT injection. Error bars, standard error of the mean (s.e.m.). ${ }^{* *} \mathrm{p}=0.007$, two-sided Mann-Whitney U test.

(D) Representative breathing airflow traces of a PSN6-DTR mouse before and after DT injection. Note that in the same period, there are 16 breaths before injection and 13 breaths after injection. Insp, inspiration; Exp, expiration. Dashed lines, zero flow.

(E) Overlay of 10 breaths from each of the traces in D, aligned to the onset of inspiration. Red traces, average of the 10 breaths; light blue, inspiratory phase. Note lengthened inspiratory time (Ti) after PSN6 ablation.

$(F, G)$ Inspiratory time (Ti) and peak inspiratory flow (PIF) changes after PSN6 ablation. Each bar represents the mean ratio of Ti or PIF distribution mode post- to pre-DT injection. Note 
increase in Ti (F) but not PIF (G) following PSN6 ablation. Error bars, error of the mean (s.e.m.). ** $\mathrm{p}=0.0036$, two-sided Mann-Whitney U tests.

(H) Inflation challenge assay. Open-chest mice were intubated and ventilated. Airflow was monitored by spirometry, and inspiratory drive was measured by electromyogram (EMG) recording of the costal diaphragm.

(I) Representative traces of integrated airflow (i.e., tidal volume) and diaphragm EMG from a control mouse during sustained expiratory (at $2 \mathrm{~cm} \mathrm{H}_{2} \mathrm{O}$ ) and inspiratory (at $8 \mathrm{~cm}$ and $10 \mathrm{~cm} \mathrm{H}_{2} \mathrm{O}$ ) holdings ( $5 \mathrm{~s}$ each, green shadings). Note decrease in the rate of EMG bursts along with increase in the pressure maintained during inspiratory holdings, indicating normal Hering-Breuer reflex. Red boxes, single bursts of EMG activity enlarged in (J).

(J) Representative traces of single diagram EMG activity bursts from control and PSN6-DTR mice after DT injections. Note longer inspiratory time (period of a single EMG burst) at $8 \mathrm{~cm}$ $\mathrm{H}_{2} \mathrm{O}$ in PSN6 ablated mouse than in control.

(K) Quantification of reflex time index (T.index) in control and PSN6-DTR mice after DT injections. T.index is the average length of EMG bursts during inspiratory holding at a given pressure normalized to that during expiratory holding for each mouse. Note lengthened inspiratory time in PSN6 ablated mice presents only at low pressures. $\dagger \mathrm{p}=0.073,{ }^{*} \mathrm{p}=0.014$, Twoway ANOVA with Sidak's multiple comparison correction. 
Figure 6. Ablation of PSN7 neurons increases tidal volume and inspiratory flow.

(A) Tidal volume (TV) changes after PSN7 ablation. Each bar represents the mean ratio of TV distribution mode post- to pre-DT injection. Error bars, standard error of the mean (s.e.m.).

** $\mathrm{p}=0.003$, two-sided Mann-Whitney U test.

(B) Representative breathing flow traces of a PSN7-DTR mouse before and after DT injection. Insp, inspiration; Exp, expiration. Dashed line, zero flow.

(C) Overlay of 10 breaths from each of the traces in B, aligned to the onset of inspiration. Red traces, average of the 10 breaths. Black dashed line, zero flow; red dashed lines, average peak inspiratory flow. Note increase in peak inspiratory flow (PIF) after PSN7 ablation (right panel). (D, E) Inspiratory time (Ti) and peak inspiratory flow (PIF) changes after PSN7 ablation. Each bar represents the mean ratio of Ti or PIF distribution mode post- to pre-DT injection. Error bars, standard error of the mean (s.e.m.). Note increase in PIF (E) but not Ti (E) following PSN7 ablation. ${ }^{*} \mathrm{p}=0.0071$, two-sided Mann-Whitney U tests. Note increase in PIF (E) but not Ti (E) following PSN7 ablation.

(F) Quantification of T.index in control and PSN7-DTR mice after DT injections. Note no change in inflation induced shortening of inspiratory time in PSN7 ablated mice. 


\section{Figure 7. Summary of the molecular, anatomical, and functional diversity of vagal PSNs}

(A) Chart summarizing the molecular, anatomical, and functional diversity of the newly defined PSN subtypes integrated with prior studies. NC, neural crest lineage, NP: neural placode lineage, (P): predicted. Physiological outputs of PSN1 and PSN2 are inferred by combining molecular identities recognized in this study and previous functional studies (Chang et al., 2015b; Han et al., 2018). Little is known about the perceptions generated by each PSN subtype.

(B) Diagram of the anatomy, sensory functions, circuits, and physiological outputs of PSN6 and PSN7 neurons. PSN6 neurons terminate on airway smooth muscle, detect airway stretch during inflation, and their activation shortens inspiratory time. PSN7 neurons terminate atop NEBs on the luminal side, and their activation limits inspiratory flow. It is likely that PSN7 neurons detect airflow or flow-associated shear stress. Since the physiological outputs of PSN6 and PSN7 are different, they presumably synapse with separate populations of second-order neurons in the nucleus of the solitary tract (NTS). VG, vagal ganglia.

(C) Comparison of pulmonary and cutaneous mechanoreceptors. The number of molecular subtypes of putative vagal pulmonary mechanoreceptors is comparable to that of cutaneous mechanoreceptors, and in both lung and skin the subtypes differ in their terminal morphologies, conduction velocities (blue highlight), adaptation properties, and the mechanical stimuli they are tuned to. Pulmonary mechanoreceptors play highly specific and critical roles in physiological reflexes and homeostasis and are capable of extensive interactions with local cells including lung resident immune cells but generate only vague perceptions. In contrast, cutaneous mechanoreceptors generate vivid perceptions of tactile quality and intensity but induce limited reflex behaviors, and their interactions with local cells in the skin appears limited but has just begun to be explored (Zhang et al., 2021). 
Figure S1. Diversity of vagal PSNs and scRNA-seq, related to Introduction and Figure 1

(A) Schematic of vagal PSNs highlighting different aspects of their diversity. Vagal sensory neurons are pseudounipolar cells, with one axonal branch projecting to an internal organ and the other projecting to the brainstem. PSNs (green) account for $\sim 15 \%$ of vagal sensory neurons, and they are heterogeneous in many ways. These include the stimuli they detect, the morphology and distribution of their termini ("receptive field"), their activity pattern in response to a given stimulus, action potential conduction velocity (correlated with myelination state), molecular identity, developmental lineage, central connectivity, physiological output, and the generated perception. FNE, free-nerve endings; ASM-T, airway smooth muscle associated terminals; NEBT, NEB-innervating terminals; Vagal Gang., vagal ganglion (nodose and jugular ganglia).

(B) Quantification of WGA-labeled neurons in vagal (VG) and dorsal root ganglia (DRG, C7-T6 collectively) on the side of the body indicated (L, left, $\mathrm{R}$, right). Dots, values from individual mice; bars, mean values.

(C) Vagal ganglion section of a Phox $2 b-C r e ; A i 32 /+$ mouse with WGA labeling from the lung, immunostained for indicated markers. Close-ups of boxed areas are shown in split panels at right. Box 1, a CGRP ${ }^{+}$Phox2b-lineage negative (EYFP') WGA-labeled neuron. Box 2, a CGRP ${ }^{+}$ Phox2b-lineage positive $\left(\mathrm{EYFP}^{+}\right.$) WGA-labeled neuron (arrowhead). Scale bars: $100 \mu \mathrm{m}, 20 \mu \mathrm{m}$ (insets).

(D) Proportion of WGA-labeled vagal sensory neurons with indicated combinations of CGRP and Phox $2 \mathrm{~b}$-lineage positivity ( $\mathrm{n}=1122$ scored neurons from 3 ganglia in 3 mice).

(E) Brightfield image of a PSN with satellite glial cell(s) still attached (arrowhead) after ganglion cell dissociation. Scale bar: $10 \mu \mathrm{m}$. 
(F) PC plots showing 7 cells (black) separated from others (gray) when satellite glial cellenriched genes were used for analysis. These cells expressed high levels of glial cell marker genes Fabp7 and Plp1. Expression scale: $\log _{2}(\mathrm{TPM}+1)$ for range indicated. Arrowhead, cell shown in (E).

(G) Sequencing readout of housekeeping genes (Gapdh, Actb) and sensory neuron marker gene (Slc17a6) across all profiled cells that passed quality control. High consistency and no drop-out indicate high quality expression profiles.

(H) Sequencing readout of tdTomato expression in cells picked from mice with genetic labeling as indicated. Red and black dots, cells with (red) and without (black) tdTomato fluorescence during cell picking. Note sequencing readout was consistent with the fluorescence records. Low levels of tdTomato reads detected in some of the fluorescence-negative cells are likely due to either low level leaky expression of the reporter gene or contaminating mRNA released from dying or dead tdTomato-expressing cells, since it was not observed in neurons from wild type mice. 
Figure S2. Molecular markers and abundances of PSN subtypes, related to Figure 2

(A) Heatmap showing relative expression levels of 260 subtype-enriched genes across the 10 molecular subtypes. Genes were selected using single cell differential expression (SCDE) method (see STAR Methods). Z scores were calculated for individual genes across all cells.

(B) Dot plot showing expression levels of genes previously identified as markers of subsets of vagal PSNs. Genes in red and orange are among the subtype-enriched genes identified here for single subtypes, with genes in red among the best markers.

(C, D) smFISH of the indicated differentially-expressed genes on mouse vagal ganglion sections with WGA retrograde labeling from the lung. Close-ups of boxed areas are shown in insets with channels split; outlined neurons are examples of indicated PSN subtypes. Scale bars: $20 \mu \mathrm{m}, 10$ $\mu \mathrm{m}$ (insets).

(E) Quantification of smFISH and immunostaining results for indicated PSN subtypes using probes/antibodies for the subtype selective genes indicated. *, likely lower than the true fraction of PSN-H neurons since Npy2r is expressed in a subset of these neurons. ${ }^{* *}$, likely higher than the true fraction of PSN3 neurons for the same reason. 
Figure S3. Terminal locations and morphologies of PSN subtypes, related to Figure 2

(A) Labeling strategy for PSN subtype neurons. AAVs expressing Cre-dependent reporter genes (fluorescent proteins or alkaline phosphatase) were injected into the vagal ganglia (1) or instilled into the lungs (2) of mice expressing Cre recombinase driven by a gene (promoter of gene $\mathrm{X}$ in diagram) selectively expressed by the subtype(s) of interest.

(B-F) Immunostaining of lung sections from $\operatorname{Trp} v 1^{\mathrm{Cre} /+}$ mice injected with AAV-CAG-DIOtdTomato into the vagal ganglia. $\mathrm{B}, \mathrm{A} \mathrm{SP}^{+}$tdTomato ${ }^{+}$fiber (PSN1) runs beneath the airway smooth muscle stained for smooth muscle actin (SMA). Note gaps between smooth muscle fibers on conducting airways, which likely allow sensing of environmental chemicals just penetrating epithelium or molecules secreted by epithelial cells. e, epithelium (outlined); al, airway lumen. $\mathrm{C}, \mathrm{A} \mathrm{SP}^{+}$tdTomato $^{+}$fiber (PSN1) terminates on a pulmonary blood vessel (bv). ca, conducting airway. Panels (right), close-up of boxed region. D, A SP ${ }^{+}$tdTomato $^{+}$fiber (PSN1) runs outside vascular smooth muscle. bvl, blood vessel lumen. E, A SP`tdTomato ${ }^{+}$fiber (PSN2, H or 3) terminates on a pulmonary blood vessel (endothelial cells stained by Pecam, blue). Panels (right), close-up of boxed region. F, A rare example of an alveolar terminal (likely PSN2) elaborating on the surface of an air sac. SP, substance P. Scale bars: $20 \mu \mathrm{m}(\mathrm{B}), 100 \mu \mathrm{m}(\mathrm{C}), 20 \mu \mathrm{m}(\mathrm{C}$ inset), $5 \mu \mathrm{m}(\mathrm{D}), 50 \mu \mathrm{m}(\mathrm{E}), 10 \mu \mathrm{m}$ (E inset), $10 \mu \mathrm{m}(\mathrm{F})$.

$(\mathrm{G}, \mathrm{H})$ Whole-mount alkaline phosphatase (AP) staining of lung lobes from $N p y 2 r^{\text {ires-Cre/+ }}$ mice intratracheally instilled with the Cre-dependent AP virus. Note in $\mathrm{H}$, all branches of the labeled PSN2 fiber terminate in the alveolar region and not the conducting airways. Scale bars: $1 \mathrm{~mm}$ (G), $0.1 \mathrm{~mm}(\mathrm{H})$.

(I) Dot plot showing expression patterns of Mc4r and PSN6 marker Lmcdl across PSN subtypes. 
(J) smFISH of PSN6 subtype marker Lmcd1 with mCherry and WGA double immunostaining on a vagal ganglion section from a $M c 4 r^{2 \mathrm{~A}-\mathrm{Cre} /+}$ mouse injected with AAV-Syn-DIO-hM3DqmCherry into the ganglion. Close-up of the boxed area is shown in right panels with channels split; outlined neuron is an example of labeled PSN6 neuron. Scale bars: $50 \mu \mathrm{m}, 10 \mu \mathrm{m}$ (inset).

(K) Quantification of PSN6 labeling specificity (serial sections of two ganglia from two $M c 4 r^{2 \mathrm{~A}-}$ ${ }_{\mathrm{Cre} /+}$ mice). Lmcd1- labeled neurons are likely PSN5 neurons.

(L) Locations of labeled termini in $M c 4 r^{2 \mathrm{~A}-\mathrm{Cre} / \mathrm{+}}$ mice injected with reporter virus into the ganglia. Quantifications were done by examining all vibratome sections of 4 entire left lobes from 4 mice. ASM, airway smooth muscle; BP, branch point.

(M) Immunostaining of PSN7 marker Calb1 with mCherry and WGA on a vagal ganglion section from a Calb $1^{2 \mathrm{~A}-\mathrm{dgCre} /+}$ mouse injected with AAV-Syn-DIO-hM3Dq-mCherry into the ganglion. Close-up of the boxed area with a labeled PSN6 neurons is shown in right panels with channels split. Scale bars: $50 \mu \mathrm{m}, 10 \mu \mathrm{m}$ (inset).

(N) Quantification of PSN7 labeling specificity (serial sections of 3 ganglia from 2 Calbl $^{2 \mathrm{~A}-}$ dgCre/+ mice).

$(\mathrm{O}, \mathrm{P})$ Whole-mount AP staining of right cranial lung lobes from $M c 4 r^{2 \mathrm{~A}-\mathrm{Cre} /+}$ (left) and Calb $1^{2 \mathrm{~A}-}$ dgCre/+ (right) mice injected with AAV-CMV-FLEX-PLAP into the vagal ganglia. Asterisks mark termini in each lobe. Insets are close-ups of boxed regions showing terminal morphology. Scale bars: $1 \mathrm{~mm}, 0.1 \mathrm{~mm}$ (insets).

(Q) Method of calculating proximal-distal (P-D) position of PSN termini. A line between the primary bronchus entry point into the lobe and a given terminal was drawn and extended to the 
edge of the lobe. Terminal P-D position is the ratio of the segment length between the entry point and the terminal to the total length of the line.

(R) Kernel density plot showing distributions of PSN6 (blue) and PSN7 (red) terminal P-D positions ( $\mathrm{n}=29$ PSN6 termini, $\mathrm{n}=36$ PSN7 termini). Note PSN7 termini are distributed more proximally than PSN6 termini.

(S, T) Close-ups from whole-mount AP staining showing termini of single PSN6 (S) and PSN7 (T) fibers. Individual termini are numbered. Scale bar: $0.1 \mathrm{~mm}$. We cannot exclude the possibility that these axons also terminate in other lung lobes or organs. 
Figure S4. Functional receptors expressed in PSN subtypes, related to Figure 3

(A) Dot plot showing expression patterns of nicotinic acetylcholine receptor subunits across PSN subtypes.

(B) Summary of predicted physiological sensing and neuronal signaling of the PSN subtypes not shown in Figure 3D.

(C) Expression of entry receptors for respiratory viruses and intracellular nucleic acid sensors (magenta) across PSN subtypes. A, adenovirus; M, measles; P, parechovirus; C-SARS, SARScoronavirus; I, influenza; RSV, respiratory syncytial virus; VS, vesicular stomatitis virus; MR, mammalian orthoreovirus; AAV, adeno-associated virus; HSV, herpes simplex virus. 


\section{Figure S5. Local interactions between lung cells and PSNs, related to Figure 4}

Predicted bi-directional cytokine signaling interactions between lung cells and vagal PSNs, inferred from expression of cytokine genes and the cognate receptor genes (connected by lines, arrows at top show the signaling direction). Full list of ligands and receptors screened is in Table S5, and genes expressed in $>50 \%$ of neurons and a mean $\log _{2}(\mathrm{TPM}+1)>1$ in at least one PSN subtype are shown here. Dashed lines divide lung immune (right side) from non-immune (left) cell types (left dot plots), and Group I (left) from Group II (right) PSN subtypes (right dot plots). AT1, alveolar type 1 cells; AT2, alveolar type 2 cells; Ba, basal cells; Cil, ciliated cells; Club, club cells; PNEC, pulmonary neuroendocrine cells; Cap, general capillary endothelial cells (gcap); Cap-a, capillary aerocytes; Artery, arterial endothelial cells; Vein, venous endothelial cells; Lymp, lymphatic endothelial cells; AlvF, alveolar fibroblasts; MyoF, myofibroblasts; Peri, pericytes; AdvF, adventitial fibroblasts; SM, smooth muscle cells; Glial, peripheral glial cells; Meso, mesothelial cells; Chondro, Chondrocytes; mDC1, myeloid dendritic type 1 cells; mDC2, myeloid dendritic type 2 cells; Ccr7+ DC, Ccr7+ dendritic cells; NC Mono, non-classical monocytes; inter Mono, intermediate monocytes; C Mono, classical monocytes; B, B cells; CD4+ T, CD4+ T cells; CD8+ T, CD8+ T cells; NK, natural killer cells; Neu, neutrophils; Bas, Basophils; InterM $\phi$, interstitial macrophages; AlvM $\phi$, alveolar macrophages. 
bioRxiv preprint doi: https://doi.org/10.1101/2021.11.10.468116; this version posted November 13,2021 . The copyright holder for this preprint (which was not certified by peer review) is the author/funder. All rights reserved. No reuse allowed without permission.

Liu et al., p. 66

Figure S6 PSN sensing inflammation and interacting with lung immune cells, related to

\section{Figure 4}

Summary of inflammatory mediators detected (blue) and immune cell-signaling cytokines secreted (magenta molecules) by the 6 PSN subtypes not shown in Figure 4C. 
Figure S7. PSN6 and PSN7 ablation and breathing changes, related to Figure 5 and 6

(A) smFISH of PSN6 subtype marker Lmcdl with GFP and WGA double immunostaining on a thin vagal ganglion section from a $M c 4 r^{2 \mathrm{~A}-\mathrm{Cre} /+}$ mouse injected with AAV-CBA-FLEX-DTRGFP into the ganglia. WGA was instilled into the lung to label PSNs. Panels at right are split channels of close-up of boxed region showing two DTR-GFP expressing PSN6 neurons. Arrowhead, another DTR-GFP expressing PSN6 neuron. Scale bars: $50 \mu \mathrm{m}$ and $10 \mu \mathrm{m}$ (inset). (B) Quantification of the percentage of $\mathrm{Lmcdl}^{+} \mathrm{WGA}^{+}$PSN6 neurons expressing DTR-GFP in PSN6-DTR mice $\left(\mathrm{n}=57 \mathrm{Lmcdl}^{+} \mathrm{WGA}^{+}\right.$neurons scored from 3 ganglia in 3 mice, with each dot representing the result from all serial sections of one ganglion).

(C) Immunostaining for GFP and WGA in a ganglion section from a PSN6-DTR mouse three days after DT injection.

(D) Quantification of DTR-GFP expressing neurons in vagal ganglia of PSN6-DTR mice before and after DT injection (each dot represents result from all serial sections of one ganglion).

(E) Selection of quiet breathing periods. Representative airflow traces during actively moving (left) or quiet (right) phases, recorded by whole body plethysmography. Portions of traces highlighted by red boxes are enlarged below. PIF.change is the absolute difference of peak inspiratory flows of two adjacent breaths. Mean PIF.change is the average PIF.change in one minute period. Note large difference in this parameter between active $(1.2 \mathrm{ml} / \mathrm{s})$ and quiet $(0.3$ $\mathrm{ml} / \mathrm{s}$ ) breathing phases. Dashed lines, zero flow; Insp, inspiration; Exp, expiration.

(F) Kernel density plot showing distribution of mean PIF.change for every minute over the entire recording period. Time periods with mean PIF.change less than twice the PIF.change at the first peak (a) were selected as quiet breathing periods. 
(G-I) Ratios of expiratory time (Te, panel G), breathing frequency (F, panel H) and minute ventilation (MV, panel I) distribution modes after DT injection (post) to before DT injection (pre) in control and PSN6-DTR mice.

(J) Reflex frequency index (F.index) in control and PSN6-DTR mice after DT injection. F.index is the frequency of EMG bursts during inspiration holding (at the indicated pressure) normalized to that during expiration holding $\left(2 \mathrm{cmH}_{2} \mathrm{O}\right)$ for each mouse.

(K) Ventilation target pressures assessed by stable EMG activity of diaphragm in control and PSN6-DTR mice. All mice were initially ventilated at $10 \mathrm{cmH}_{2} \mathrm{O}$ target pressure, which completely suppressed diaphragm EMG activity. Target pressure was then gradually reduced until diaphragm resumed rhythmic EMG activity.

(L) Immunostaining for PSN7 subtype marker Calb1 along with GFP and WGA on a thin vagal ganglion section from a Calb1 $1^{2 \mathrm{~A}-\mathrm{dgCre} /+}$ mouse injected with AAV-CGA-FLEX-DTR-GFP into the ganglion and treated with TMP. WGA was instilled into the lung to label PSNs. Arrowheads, DTR-GFP expressing PSN7 neurons. Scale bar: $50 \mu \mathrm{m}$.

(M) Quantification of the percentage of Calb1 $1^{+} \mathrm{WGA}^{+}$PSN7 neurons expressing DTR-GFP in PSN7-DTR mice $\left(\mathrm{n}=95 \mathrm{Calb}^{+} \mathrm{WGA}^{+}\right.$neurons scored from 4 ganglia of 2 mice, with each dot representing result for all serial sections of one ganglion).

(N) Immunostaining for GFP and WGA on a ganglion section from a PSN7-DTR mouse three days after DT injection.

(O) Quantification of DTR-GFP expressing PSNs in vagal ganglia of PSN7-DTR mice before (pre) and after (post) DT injection (each dot represents result from all serial sections of one ganglion). 
bioRxiv preprint doi: https://doi.org/10.1101/2021.11.10.468116; this version posted November 13,2021 . The copyright holder for this preprint (which was not certified by peer review) is the author/funder. All rights reserved. No reuse allowed without permission.

Liu et al., p. 69

(P-S) Ratios of peak expiratory flow (PEF, panel P), expiratory time (Te, panel Q), breathing frequency $(\mathrm{F}$, panel $\mathrm{R})$ and minute ventilation (MV, panel S) distribution modes after DT injection (post) to before DT injection (pre) in control and PSN7-DTR mice.

(T) Reflex frequency index (F.index) in control and PSN7-DTR mice after DT injection. 


\section{RESOURCE AVAILABILITY}

\section{Lead contact and materials availability}

Further information and requests for resources and reagents should be directed to the Lead Contact, Mark A. Krasnow (krasnow@stanford.edu). All unique/stable reagents generated in this study are available from the Lead Contact upon proper request.

\section{EXPERIMENTAL MODEL AND SUBJECT DETAIL}

\section{Animals}

Mouse lines used in this study are listed in the Key Resources Table. Wild type (C57BL/6) mice were obtained from Jackson Laboratories. All studies were carried out on a mixture of adult (at least 6-week-old) male and female mice, with comparable numbers of each sex allocated to experimental groups. Mice were maintained in 12 hour light/dark cycle with food and water provided ad libitum. All animal husbandry, maintenance, and experiments were performed in accordance with Stanford University’s IACUC-approved protocols (APLAC 9780, 28836).

\section{METHODS DETAIL}

\section{Retrograde labeling of vagal PSNs}

Mice were anesthetized with $2.5 \sim 3 \%$ isoflurane in induction chamber for at least 15 min and then placed on an intubation stand (Rodent Tilting Workstand, Hallowell EMC). Light from a microscope illuminator was placed gently against the neck. The mouse's mandible was lifted, and tongue was extended with a Q-tip. Vocal cord was visualized with a speculum and an intubation tube (1 inch $20 \mathrm{G}$ disposable catheter, Fisher Scientific) was inserted into the trachea with a guide wire (Mouse Intubation Pack, Hallowell EMC). After removing the guide wire, 50 $\mu 1$ of $1 \mathrm{mg} / \mathrm{ml}$ fluorescent conjugated WGA was instilled into the lung during natural breathing. For double labeling from the lung, each of the fluorescent conjugated WGAs (Alexa488 and 
Alexa555) was instilled separately one day apart. Two other commonly used tracers, $\operatorname{DiIC}_{18}(3)$ (1 $\mathrm{mg} / \mathrm{ml}$ in 10\% DMSO/90\% saline, sonicated) and fluorescent conjugated CTB $(1 \mathrm{mg} / \mathrm{ml})$, were also tested, but they labeled fewer neurons than WGA (data not shown).

\section{Retrograde labeling of vagal stomach sensory neurons}

Mice were anesthetized with $1.5 \%$ isoflurane via nose cone (after induction with $2-3 \%$ isoflurane). A horizontal incision was made in abdominal skin, followed by an incision in the abdominal muscle to expose the stomach. Fluorescent conjugated WGA ( $5 \mathrm{ul} \mathrm{of} 5 \mathrm{mg} / \mathrm{ml}$ solution) was injected into the wall of the dorsal stomach ( $3 \sim 6$ sites) using glass pipettes connected to an aspirator tube (Sigma, A5177).

\section{Single cell transcriptional profiling}

Mice used for collecting single PSNs for transcriptional profiling were adults 6-12 weeks old. Vagal ganglion dissociation

Our protocol was modified from one previously described (Dubuis et al., 2013). Only one mouse was used for dissociation each day (two ganglia yield enough cells for picking, and the source animal for each cell was recorded so that transcriptomic variance introduced by individual mice could be analyzed and excluded during cluster analysis). Four to seven days after retrograde labeling, mice were euthanized with $\mathrm{CO}_{2}$ and transcardially perfused with cold Hank's balanced salt solution (HBSS) (without $\mathrm{Ca}^{2+}$ and $\mathrm{Mg}^{2+}$ ). Vagal ganglia were quickly dissected after perfusion in cold buffered HBSS (10 mM HEPES, pH7.2-7.5) and each was cut into $2 \sim 3$ pieces. Ganglion tissue was then digested with papain solution at $20 \mathrm{U} / \mathrm{ml}$ (Worthington LS003124, in buffered HBSS containing 0.5 mM EDTA, $1.5 \mathrm{mM} \mathrm{CaCl2,} 0.4 \mathrm{mg} / \mathrm{ml}$ L-cysteine, pre-warmed for $30 \mathrm{~min}$ for activation) for $10 \mathrm{~min}$ at $37^{\circ} \mathrm{C}$, and then collagenase/dispase solution (collagenase IV, 300 U/ml, Worthington LS004186; dispase, 1mg/ml, Thermo Fisher Scientific 
$17105-041$, in HBSS, pre-warmed) for $30 \mathrm{~min}$ at $37^{\circ} \mathrm{C}$ with tube inversion to agitate every 10 min. After centrifuging at $400 \mathrm{~g}$ for $4 \mathrm{~min}$, tissue pellet was resuspended in $1 \mathrm{ml} \mathrm{L15}$ medium (with 10mM HEPES pH 7.2-7.5 and 10\% fetal bovine serum) and triturated using fire-polished Pasteur pipettes. A Percoll gradient spin was used to clear axonal debris and glial cells by layering the cell suspension onto a buffered L15 medium (10 mM HEPES)/Percoll (4:1) solution and then centrifuging for $9 \mathrm{~min}$ at $400 \mathrm{~g}$. The cell pellet was resuspended in $2 \mathrm{ml}$ buffered L15 medium, centrifuged again for $3 \mathrm{~min}$ at $750 \mathrm{~g}$, and resuspended in $200 \mu \mathrm{l}$ suspension medium (L15 medium, 10mM HEPES pH 7.2-7.5, 1mg/ml BSA, and 0.1\% Pluronic F-68). We tested this protocol for satellite glial dissociation by placing the cells onto poly-d-lysine coated cover glasses, fixing with $4 \%$ PFA in PBS, and then staining for DAPI; less than $10 \%$ of single neurons were associated with additional nuclei on their side, indicating nearly complete removal of glial cells. The protocol is optimized for the nodose side of the vagal ganglion, and the jugular side (neural crest derived) was likely over-digested; only $\sim 3 \%$ of WGA labeled cells were neural

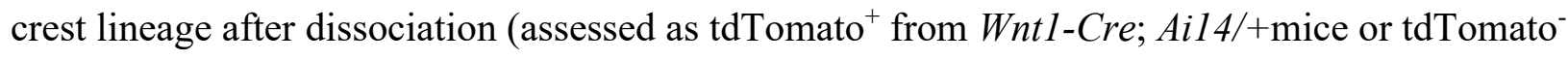
from Phox2b-Cre; Ail4/+ mice).

\section{Single cell picking}

The single cell suspension was placed on ice, and cell picking was carried out within 2 hours after completion of ganglion dissociation to maximize cell health. Drops $(2 \mu 1)$ of cell suspension were placed on a petri dish and examined with an inverted fluorescent microscope to identify WGA-488 labeled sensory neurons. Individual labeled neurons were collected using a custom glass micropipette with inside filament (WPI TW100F-4) connected to an aspirator tube (Sigma A5177). The micropipette was made with a pipette puller (Sutter instrument, P-87) and the tip fabricated by the "glass-on-glass" method (Pipette cookbook) to produce a 50-100 micron 
diameter opening. Liquid $(0.3 \mu 1$ or less $)$ containing a single labeled cell was aspirated into the pipette by capillary action and then transferred to a fresh, ice-cold $2 \mu 1$ drop of suspension medium. The cell was picked again with a fresh glass micropipette, transferred to a $0.2 \mathrm{ml} \mathrm{PCR}$ tube containing $4 \mu \mathrm{l}$ of lysis buffer, and then immediately frozen on dry ice. Transfer to fresh medium and the second round of picking was performed to ensure purity. A micrograph was taken of each cell prior to the second pick, and later used to measure cell size. Single cell lysates were stored at $-80^{\circ} \mathrm{C}$ before proceeding to cDNA synthesis and amplification.

cDNA synthesis and amplification

cDNA synthesis and amplification of single vagal PSNs were carried out using the SMARTSeq v3 Ultra Low Input RNA Kit (Clontech). To minimize amplification bias, cDNA amplification was limited to $14-15$ cycles. The concentration and quality of cDNA was examined by Bioanalyzer using the High Sensitivity DNA kit (Agilent 5067-4626), and any sample with $<30 \mathrm{pg} / \mu 1 \mathrm{cDNA}$ was discarded (1 of 137).

\section{Library preparation, sequencing, and raw data processing}

Our method for constructing sequencing libraries has been described (Ouadah et al., 2019). Briefly, amplified cDNA was diluted to $30 \sim 150 \mathrm{pg} / \mu 1$ range and followed by tagmentation and amplification with index primers using the Nextera XT DNA Library Preparation Kit (Illumina FC-131-1096) and Index Adapters (Illumina, FC-131-1002). These steps followed the C1 system for mRNA Seq Protocol (Fluidigm). Library concentration and quality was examined by Bioanalyzer using the High Sensitivity DNA kit (Agilent 5067-4626), and sequencing was carried out on NextSeq 500 (Illumina) with 75 bp paired-end reads. Raw reads were aligned to the NCBI RefSeq mouse transcriptome (modified to exclude model XR_/XM_sequences and include the tdTomato sequence) using kallisto with default settings (Bray et al., 2016). Read 
count and TPM values from splice isoforms of the same genes were collapsed, and for quality control cells with less than 2,000,000 paired-end reads that mapped to endogenous genes or less than 10,000 endogenous genes with mapped paired-end reads were excluded from the following analysis.

\section{scRNA-seq data analysis}

\section{Identification and exclusion of glial-contaminated cells}

The TPM table was log-transformed $\left(\log _{2}(\mathrm{TPM}+1)\right)$ and scaled, and PCA was performed using 305 glial cell-enriched genes (Li et al., 2016). Cells were plotted along the first two PCs and 7 cells clearly separated from the rest (Figure S1F), which were considered glialcontaminated and eliminated from the following cluster analysis.

Iterative cluster analysis

Our algorithm is modified from one previously described (Tasic et al., 2016). For each round of iterative clustering, the following steps were performed:

1. Mean expression and variance were calculated based on $\log _{2}(\mathrm{TPM}+1)$ values for all endogenous genes.

2. Highly variable genes with different combinations of mean expression (1 to 3 with 0.5 increment) and variance (1 to 3 with 0.5 increment) thresholds were selected ( 25 sets). Step 3 to step 5 were carried out for each set.

3. PCA was performed using highly variable genes (prcomp function) and significant PCs were selected using the Jackstraw package. When the number of highly variable genes was smaller than the number of cells, data was randomized for each column (cell) and PCA repeated 100 times. Eigen values of each PC from the original PCA were compared with eigen values of PC1 
from the permuted PCAs using a t-test, and PCs with multiple comparison adjusted p-value $<0.05$ were selected as significant PCs.

4. Euclidian distances were calculated using the cell loadings from the significant PCs and followed by hierarchical clustering using the "ward. D2" method.

5. Only the first split from hierarchical clustering was taken into consideration and the significance level of the split was calculated using SigClust package.

6. Among the 25 sets of highly variable genes, if no p-value from step 5 was $<0.05$, clustering was terminated.

7. If one or more $\mathrm{p}$-values from step 5 was $<0.05$, the set of highly variable genes that resulted in the lowest p-value was selected and randomly subsampled (80\%), and steps 3 to step 5 repeated 100 times. Cells that clustered into the same group in $>90 \%$ of the events were accepted as a stable cluster, whereas cells that switched between groups in $>10 \%$ of the events were labeled as outliers. When a group of cells ( $\geq 5$ cells) switched their group identity together in $>90 \%$ of the events, they were accepted as a third cluster.

8. Clusters comprising less than 5 cells or cells that all came from one animal were not promoted to the next round or accepted as final clusters.

9. After the final clusters were obtained, each cluster was cross-validated with each of the other clusters (except those that were resolved together at the final round of iteration) using step 1-7, and new outliers were identified.

\section{Identification of subtype-enriched genes}

Subtype-enriched gene analysis was carried out using the scde package with read counts table as input (Kharchenko et al., 2014). Error models for cells from different subtypes were fitted independently using the "groups" argument. For each subtype (except PSN-H), differential 
expression analysis was carried out with each of the other subtypes to generate 8 lists of upregulated genes (adjusted $\mathrm{z}$ score $>1.7$ and fold-change $>2$ ), and subtype-enriched genes were further identified by intersecting the lists. For PSN1-8, the lists of upregulated genes identified by comparison to PSN9 using scde were not used due to the low number of cells in PSN9; instead the average $\log _{2}(\mathrm{TMP}+1)$ was used, and genes expressed $\geq 2$-fold higher $\left(\Delta \log _{2}(\mathrm{TMP}+1)>1\right)$ in PSN1-8 than PSN9 were included in the list. For PSN9, the enriched genes were identified by intersecting lists of upregulated genes generated by scde from all comparisons.

Mouse lung scRNA-seq data analysis scRNA-seq data of the mouse lung was a combined dataset of two previous reports (Tabula Muris, 2018; Travaglini et al., 2020), containing 2153 cells from adult mice (postnatal 60-90 days) profiled using SMART-seq2 method. The previously assigned cell type annotations were used.

\section{Histology}

\section{Immunostaining on vagal ganglion sections}

Vagal ganglia were dissected freshly and fixed with $4 \% \mathrm{PFA}$ at $4{ }^{\circ} \mathrm{C}$ for 2 hours. After washing with PBS, ganglia were cryoprotected with $30 \%$ sucrose in PBS and embedded in optimal cutting temperature compound (OCT). Cryosections were cut at $18 \mu \mathrm{m}$ and dried overnight at room temperature. After washing $2 \times 5$ min with PBS and $1 \times 5$ min with PBS $+0.1 \%$ Triton X-100, sections were incubated with blocking solution (PBS+0.1\% Triton X-100+5\% normal donkey serum) for $30 \mathrm{~min}$ at room temperature, followed by primary antibodies at $4{ }^{\circ} \mathrm{C}$ overnight. The next day, sections were washed $3 \times 10$ min with PBS $+0.1 \%$ Triton X-100, incubated with secondary antibodies (1:500) for 45-60 min at room temperature. After washing 
again $3 \times 10$ min with PBS $+0.1 \%$ Triton X-100 and then briefly rinsing in PBS, sections were mounted with fluoromount-G. Primary antibodies used are listed in the Key Resources Table. Single molecule fluorescent in situ hybridization (smFISH) with immunostaining

Vagal ganglia from mice retrogradely labeled with WGA were mounted in OCT and freshly frozen on dry ice. Cryosections were cut at $12-14 \mu \mathrm{m}$ and post-fixed for 45-60 min to preserve the WGA throughout in situ hybridization. smFISH (RNAscope) was performed using the supplier's protocol (Advanced Cell Diagnostics, ACD), followed by the immunostaining protocol described above.

\section{Immunostaining on lung vibratome sections}

Mouse lungs were perfused with PBS through the pulmonary circulation and inflated with 2\% low-melting point agarose in PBS. After removal from the body, the inflated lungs were fixed with $4 \%$ PFA in PBS for 5 hrs at $4{ }^{\circ} \mathrm{C}$. After washing thoroughly with PBS, lung lobes (left and right cranial) were separated and glued onto the cutting stage of the vibratome, and sections (350 $\mu \mathrm{m})$ were cut parallel to the ventral surface of the lobes.

Vibratome sections were washed with PBS, blocked with PBS $+0.5 \%$ Triton X-100 $+5 \%$ normal donkey serum for 1 hour at room temperature, then incubated with primary antibodies for 2.5 days at $4{ }^{\circ} \mathrm{C}$ on a nutator/shaker. Antibody-stained sections were washed with PBS $+0.5 \%$ Triton X-100 for $2 \times 30$ min, $2 \times 1$ hour, $1 \times 2$ hours, then incubated with secondary antibodies (1:500) for 1-2 days at $4^{\circ} \mathrm{C}$ on a nutator/shaker. Sections were washed as above after secondary antibody incubation, then post-fixed with $4 \%$ PFA in PBS for $1-2$ hrs at $4{ }^{\circ} \mathrm{C}$, dehydrated with $50 \%, 75 \%$, and 100\% methanol, then cleared in BABB (Benzyl Alcohol:Benzyle Benzoate 1:2). Images were acquired with a Zeiss 780 confocal scanning microscope under 25x oil objective.

\section{Alkaline Phosphatase staining}


Mouse lungs were perfused and inflated as described above. After removal from the body, the inflated lungs were fixed with $4 \%$ PFA in PBS for 2 hours at $4{ }^{\circ} \mathrm{C}$. Whole lungs were then washed with $\mathrm{PBS}$, incubated in $\mathrm{PBS}$ at $65-68^{\circ} \mathrm{C}$ for 2 hours to inactive endogenous alkaline phosphatase, and stained with BCIP/NBT solution (diluted in $0.1 \mathrm{M}$ Tris- $\mathrm{HCl} \mathrm{pH} 9.6,0.1 \mathrm{M}$ $\mathrm{NaCl}, 50 \mathrm{mM} \mathrm{MgCl} 2,0.1 \%$ Triton X-100) overnight at room temperature. The next day, lungs were washed with PBS, post-fixed with $4 \%$ PFA in PBS overnight at $4{ }^{\circ} \mathrm{C}$, dehydrated with $50 \%$, $75 \%$, and $100 \%$ methanol, then cleared in BABB.

\section{Vagal ganglion injection}

Vagal ganglion injections were performed as previously described with minor modifications (Chang et al., 2015). Briefly, mice were intubated and kept anesthetized with $1.5 \%$ isoflurane (23\% for induction) using a low-flow anesthesia system (Somnosuite, Kent Scientific, Physiosuite modules incorporated). To avoid complications from breathing movements, mice were ventilated (RoVent module) at 90 breaths per minute (10-12 $\mathrm{cmH}_{2} \mathrm{O}$ target pressure). The vagal ganglion was exposed from the ventral side of the neck. Injections were carried out using custom glass pipettes (pulled by micropipette puller P-87, Sutter Instrument) connected to UltraMicroPump (World Precision Instruments) with Micro4 controller (World Precision Instruments). Virus solution (200 nl), containing 0.05\% Fast Green (Sigma, F7252) for visualization during injection, was injected to each ganglion at a rate of $20 \mathrm{nl}$ per minute. For histological analysis, mice injected with AAV-CAG-DIO-tdTomato or AAV-Syn-DIO-hM3Dq-mCherry were sacrificed at least 3 weeks after ganglion injection, and mice injected with AAV-CMV-DIO-PLAP were sacrificed at least 2 weeks later.

\section{Chemical delivery}

\section{Trimethoprim}


Trimethoprim (TMP, Sigma T7883) was delivered to mice by oral gavage. On each day of treatment, a $50 \mathrm{mg} / \mathrm{ml}$ stock solution was freshly prepared by dissolving TMP in DMSO followed by vigorous vortexing. The stock solution was further diluted 1:5 in sterile water and immediately delivered in the appropriate volume at a dose of $300 \mu \mathrm{g} / \mathrm{g}$ body weight.

\section{Diphtheria toxin}

Diphtheria toxin (Biological Laboratory, \#150) was dissolved in PBS to make a $20 \mu \mathrm{g} / \mathrm{ml}$ stock solution and stored at $-20^{\circ} \mathrm{C}$. On each day of treatment, stock solution was diluted in sterile saline to make a $200 \mathrm{ng} / \mathrm{ml}$ injection solution and injected i.p. at a dose of $10 \mathrm{ng} / \mathrm{g}$ body weight.

\section{Breathing assays}

\section{Whole-body plethysmography}

Whole-body plethysmography recordings were performed on unanesthetized and unrestrained mice using Buxco chambers (450 ml, Model PY4211) or Vivoflow chambers (SciReq) and data was acquired and analyzed using usbAMP and iox2 software (SciReq). For ablation experiments, each mouse was pre-acclimated to the chamber for two consecutive days (minimum 1.5 hours per day) before the first day of recording (prior to DT injection) and for one day before the post DT recording (third day post DT injection). On each recording day, mice were recorded for at least 2 hours.

To analyze respiratory parameters, breaths in the first 30 min of recordings were not used, and breaths that had a tidal volume (TV) less than $0.05 \mathrm{ml}$ or greater than $2 \mathrm{ml}$, or a peak inspiratory flow (PIF) less than $1 \mathrm{ml} / \mathrm{s}$ or greater than $25 \mathrm{ml} / \mathrm{s}$ were filtered out. To automatically select calm breaths, all breaths after filtering were segmented into 1 min windows, and the average change in PIF of every pair of consecutive breaths was calculated within all windows. The distribution of average PIF changes was calculated by the density function in $\mathrm{R}$, and all 1 minute windows in 
the first peak $(\leq 2 \mathrm{x}$ the first mode) were selected as quiet breathing periods (Figure S7E, F). Breaths in quiet breathing periods were used to plot distributions of individual parameters and calculate distribution modes or averages for comparison. Minute ventilation was calculated by summing the TV of all calm breaths then dividing by the total time (in minutes).

\section{Inflation assay}

Mice were anesthetized by two consecutive i.p. injection of $20 \%$ urethane in saline (each 1 $\mathrm{mg} / \mathrm{g}$ body weight) one hour apart, and surgery started one hour after the second injection. Additional injection(s) of 2-4 mg were given (total $<10 \mathrm{mg}$ ) as needed if the toe pinch reflex was not completely abolished. Mice were intubated with a 1 inch $20 \mathrm{G}$ disposable catheter and connected to Physiosuite (Kent Scientific) supplied with room air at 50-60 ml/min. Body temperature was kept at $36^{\circ} \mathrm{C}$ using a heating pad (RightTemp, Kent Scientific). The trachea was exposed, and a suture was used to seal the endotracheal tube airtight in the trachea. Chest and abdominal skin were incised to expose the sternum and the left side of the diaphragm. Prior to opening the chest cavity, the ventilator was started in pressure control mode (RoVent, Kent Scientific, 150 breaths/min, target pressure: $10 \mathrm{cmH}_{2} \mathrm{O}$, PEEP: $2 \mathrm{cmH}_{2} \mathrm{O}, \mathrm{I} / \mathrm{E}$ ratio: 1:1.5). The chest cavity was opened by cutting the sternum (along the midline to avoid bleeding). A two-lead stainless steel needle electrode was inserted into the left costal diaphragm, and diaphragm EMG signal was recorded with BioAmp connected to Powerlab (ADInstrument). Airflow in the ventilation circuit was monitored using a spirometer with a pneumotach flow head, and signals were recorded using Powerlab. Any mice that had diaphragm rhythmic activity when ventilating at $10 \mathrm{cmH}_{2} \mathrm{O}$, indicating an abnormal mechanoreflex, were not proceeded to inspiratory/expiratory challenge. Target pressure was then gradually lowered until diaphragm rhythmic activity initiated $\left(6 \sim 8 \mathrm{cmH}_{2} \mathrm{O}\right)$ with a frequency equal to or higher than ventilation. 
Inflation and deflation holding were delivered by Physiosuite-RoVent for $5 \mathrm{~s}$ each. Between two consecutive holding challenges, there was at least 2 min of regular ventilation. Serial challenges on the same mouse followed an order that alternated between high pressures $\left(>12 \mathrm{cmH}_{2} \mathrm{O}\right)$ and low pressures $\left(<12 \mathrm{cmH}_{2} \mathrm{O}\right)$ to avoid atelectasis throughout multiple challenge trials.

Diaphragm EMG signals were high-pass filtered at $150 \mathrm{~Hz}$. For each inspiratory challenge, average time of individual bursts of EMG activity (mean Ti) and frequency of bursts (F) were calculated, then divided by the mean Ti and F of the EMG bursts during expiratory challenge to generate a Ti.index and a F.index (if multiple expiratory challenge trials were performed, the closest trial was selected). For each mouse, if multiple trials with the same inspiratory pressure were performed then a mean Ti.index and a mean F.index were calculated for the corresponding pressure.

\section{Statistical analysis}

Sample sizes including number of ganglia and number of mice are reported for each experiment in the figures or figure legends. Statistical analysis and plotting were preformed using Graphpad Prism8.4. All bar plots show the mean values \pm SEM (error bars). Statistical methods for comparison are reported in figure legends. In figures, asterisks are used to denote significance, with $* \mathrm{p}<0.05, * * \mathrm{p}<10-2$, and $* * * \mathrm{p}<10-3$. 


\section{Figure 1}
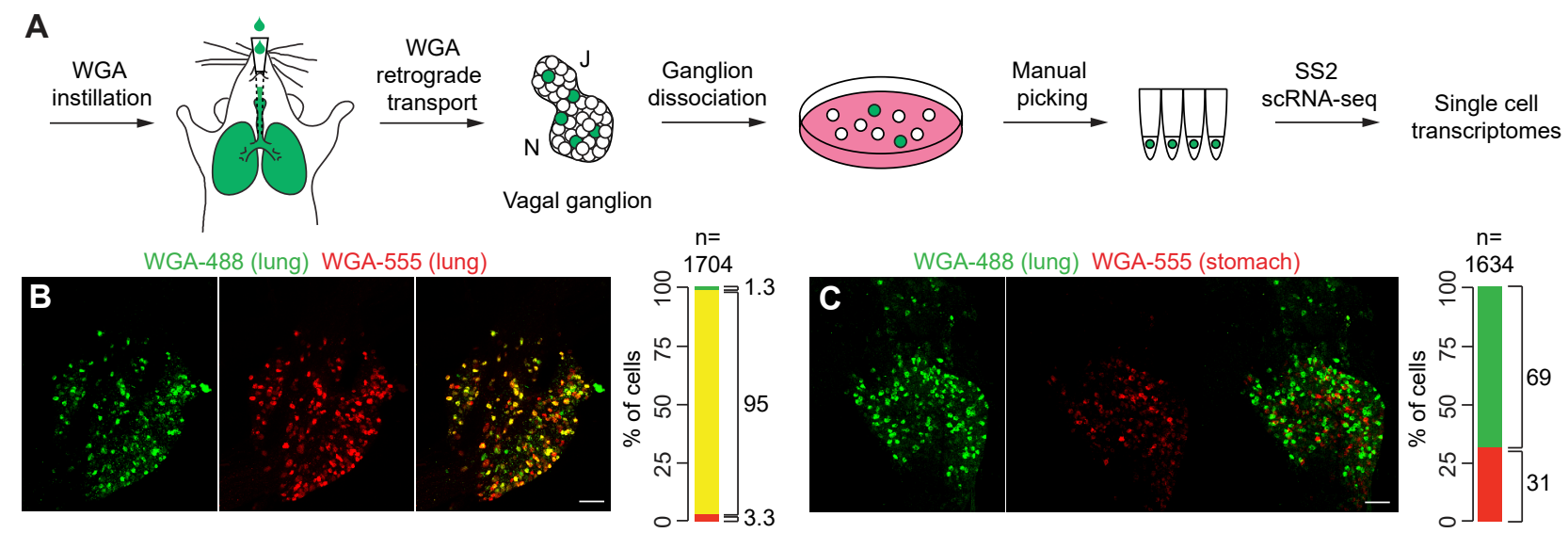

D

$$
\text { QC-qualified }
$$
single cell transcriptomes
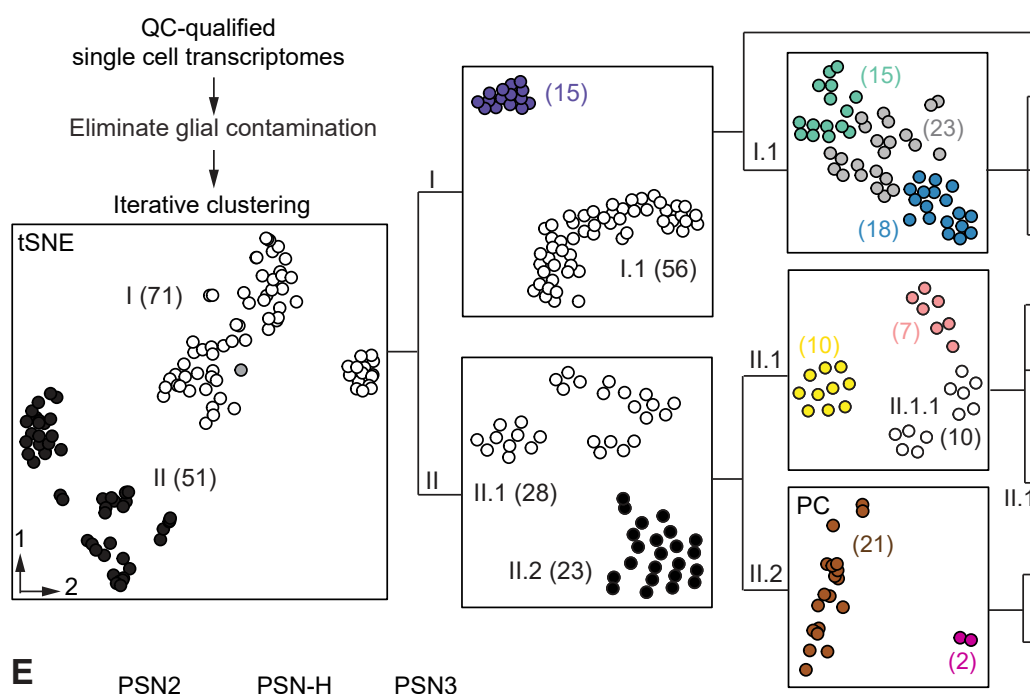

Fiber type

Dev. Lineage

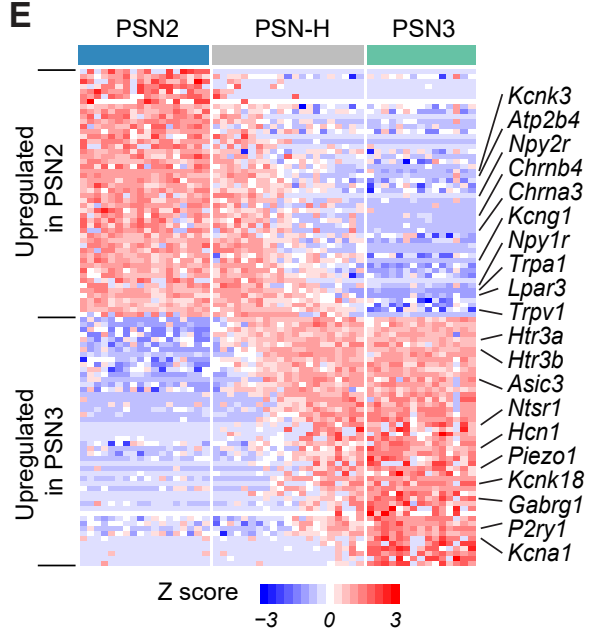

F $\quad$ G

G Nefh

H

Crest

PSN1 C

Placode

Placode

Placode

Placode

Placode

Placode

Placode

Placode

Crest
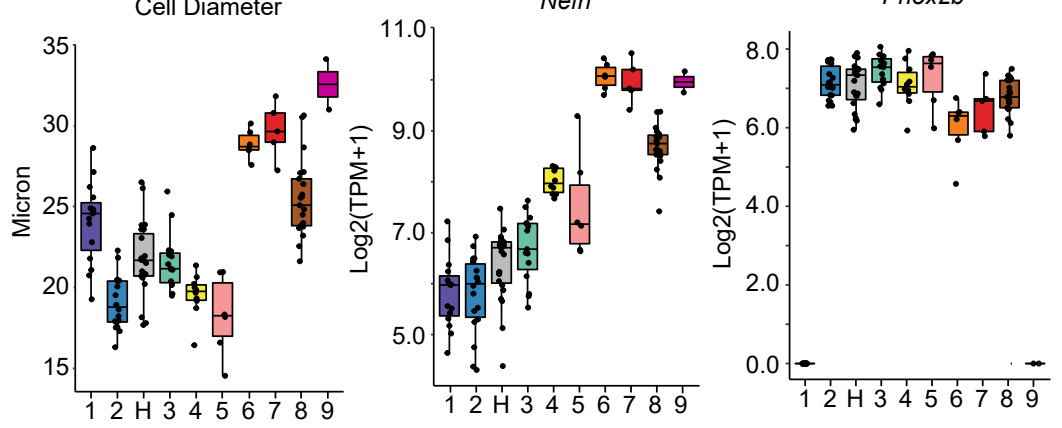


\section{Figure 2}

A
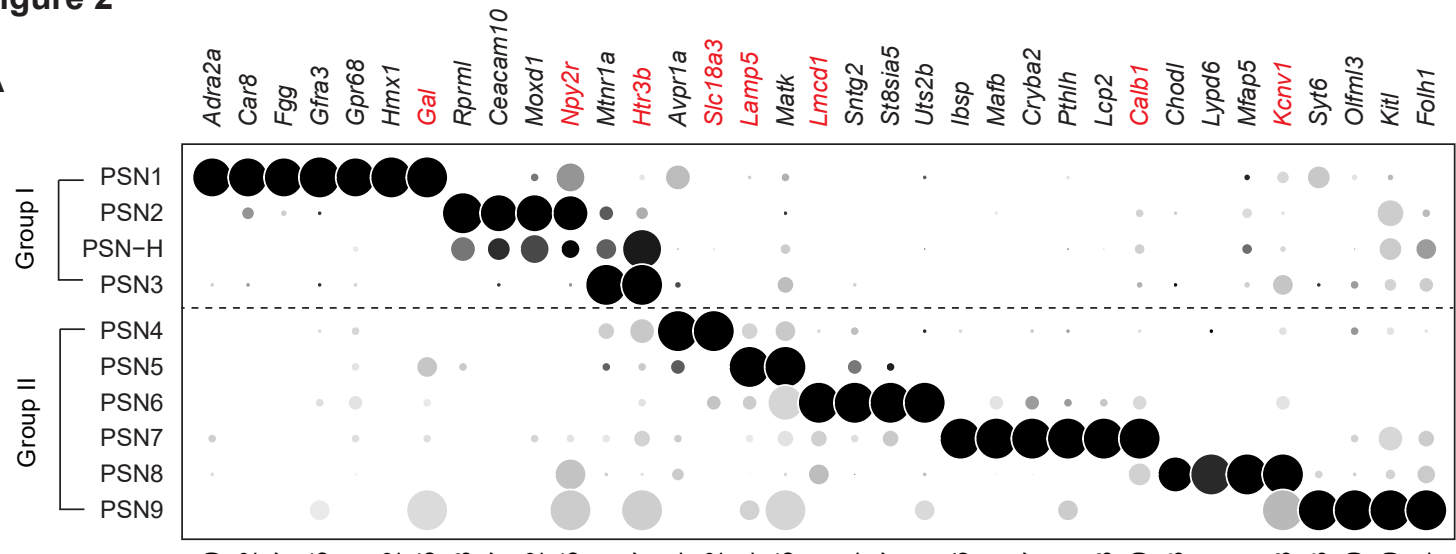

B

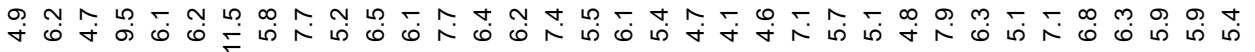
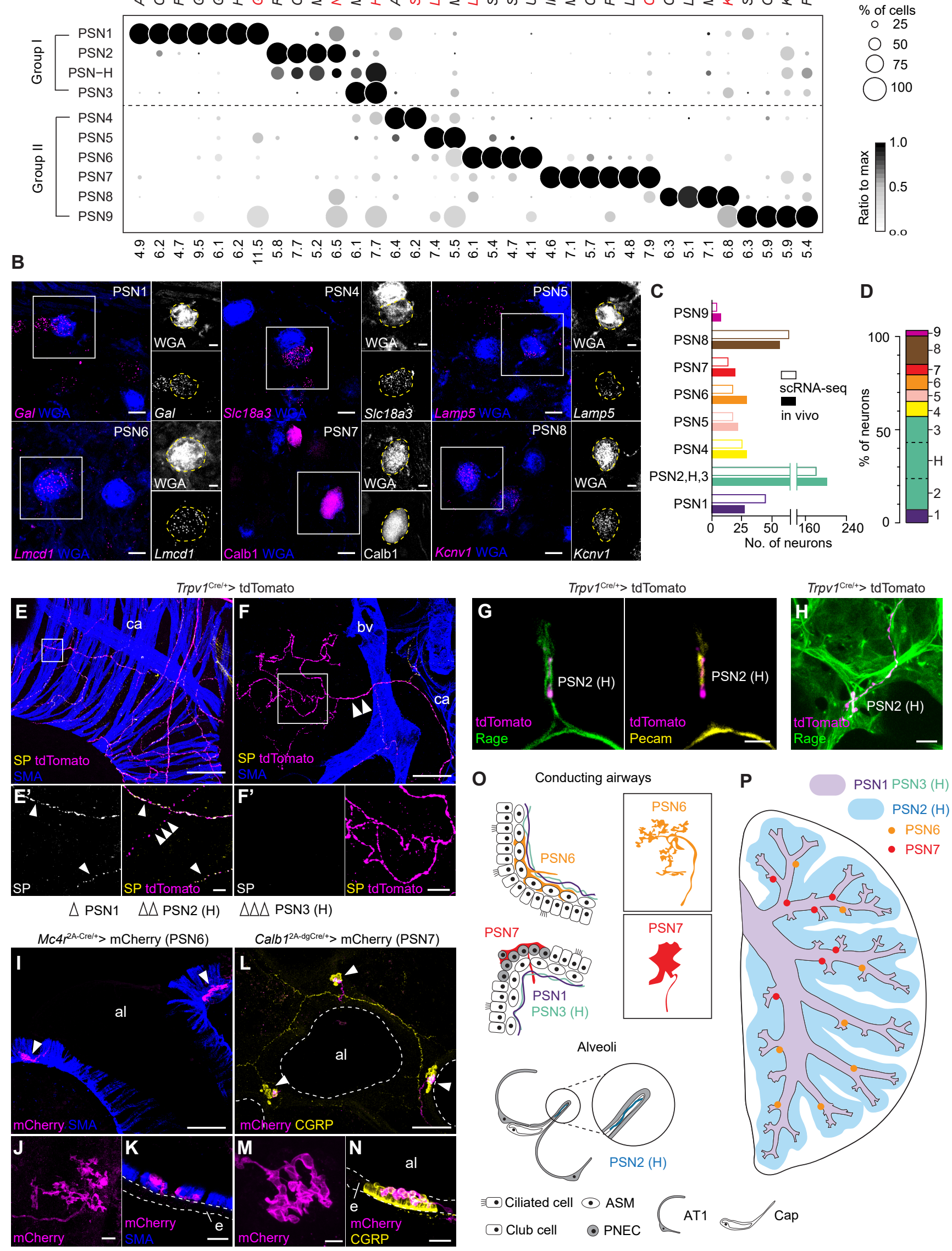
Figure 3

A

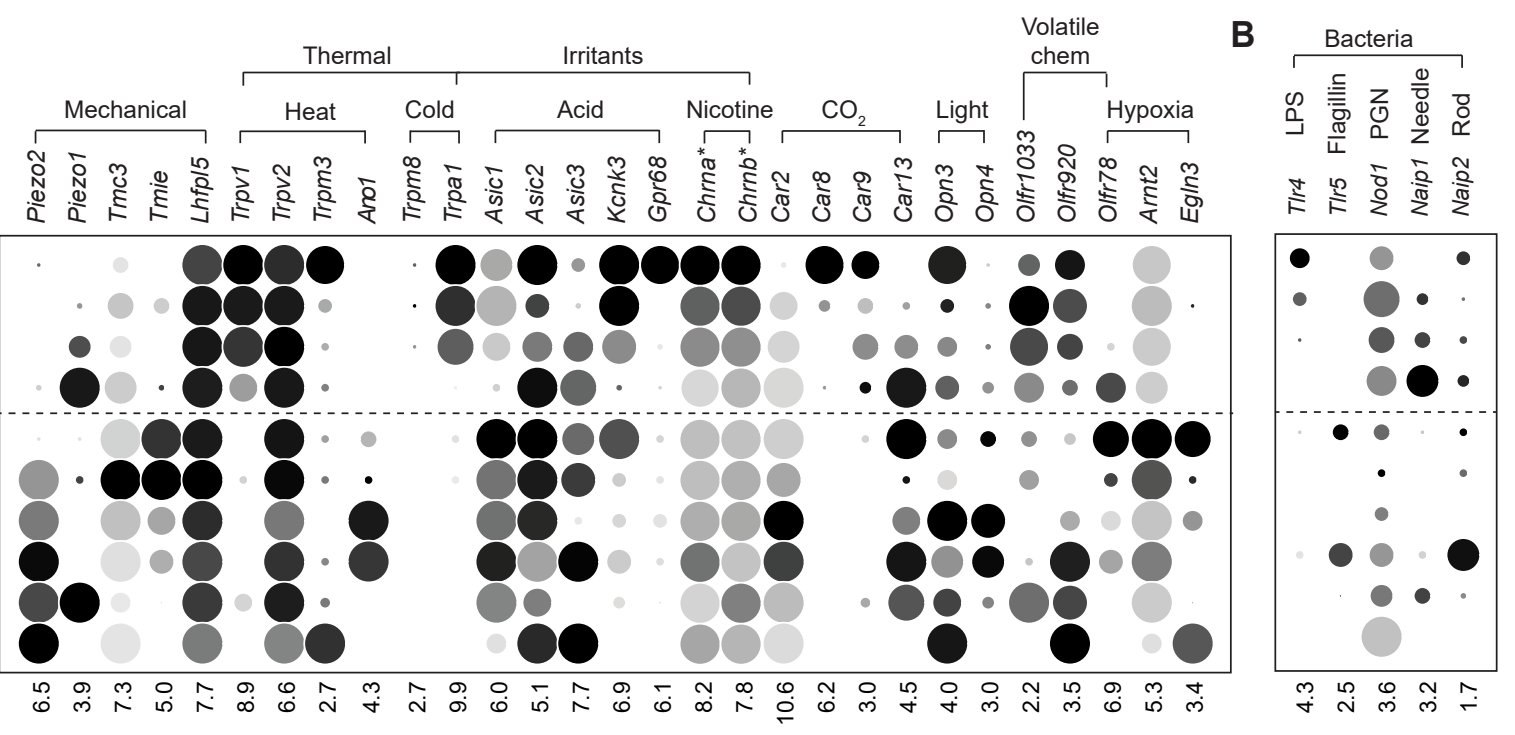

C

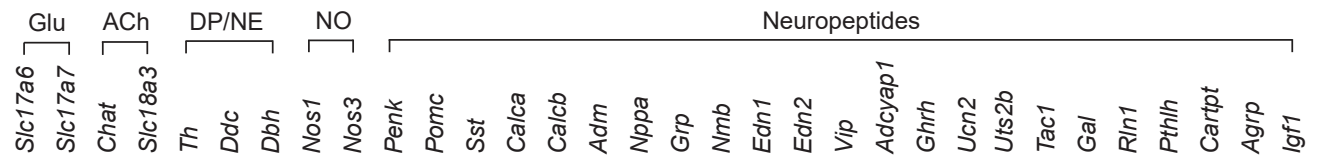

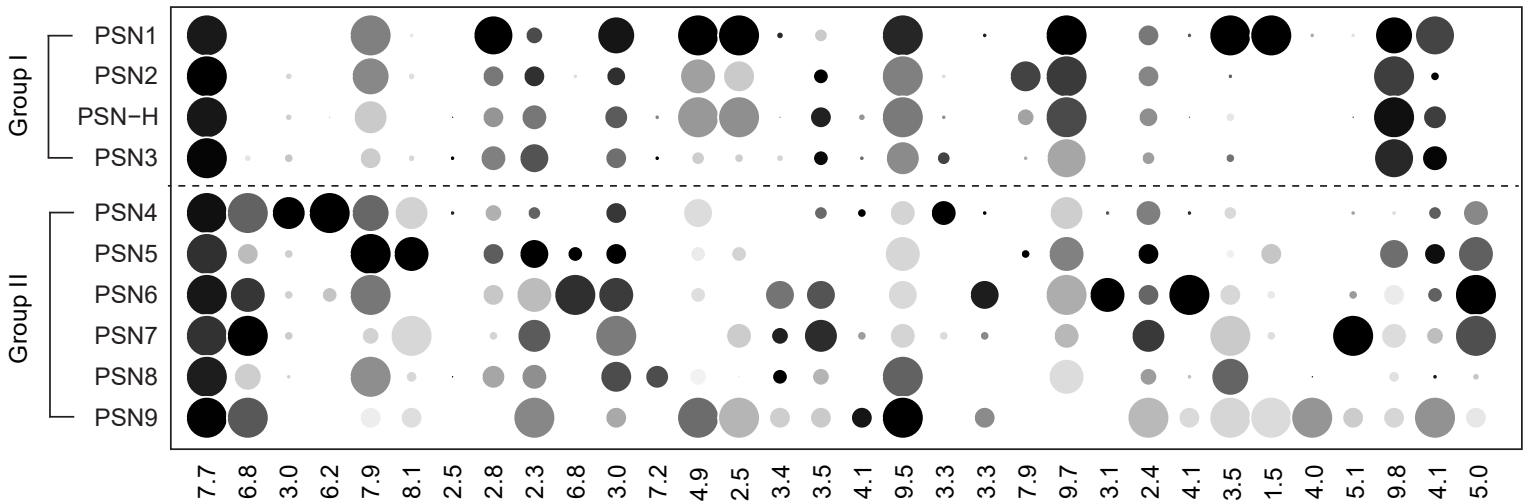

$\%$ of cells

○ 25

○ 50

75

100

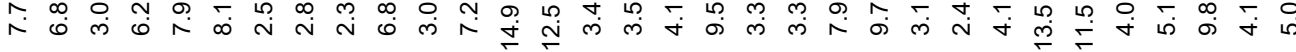

D
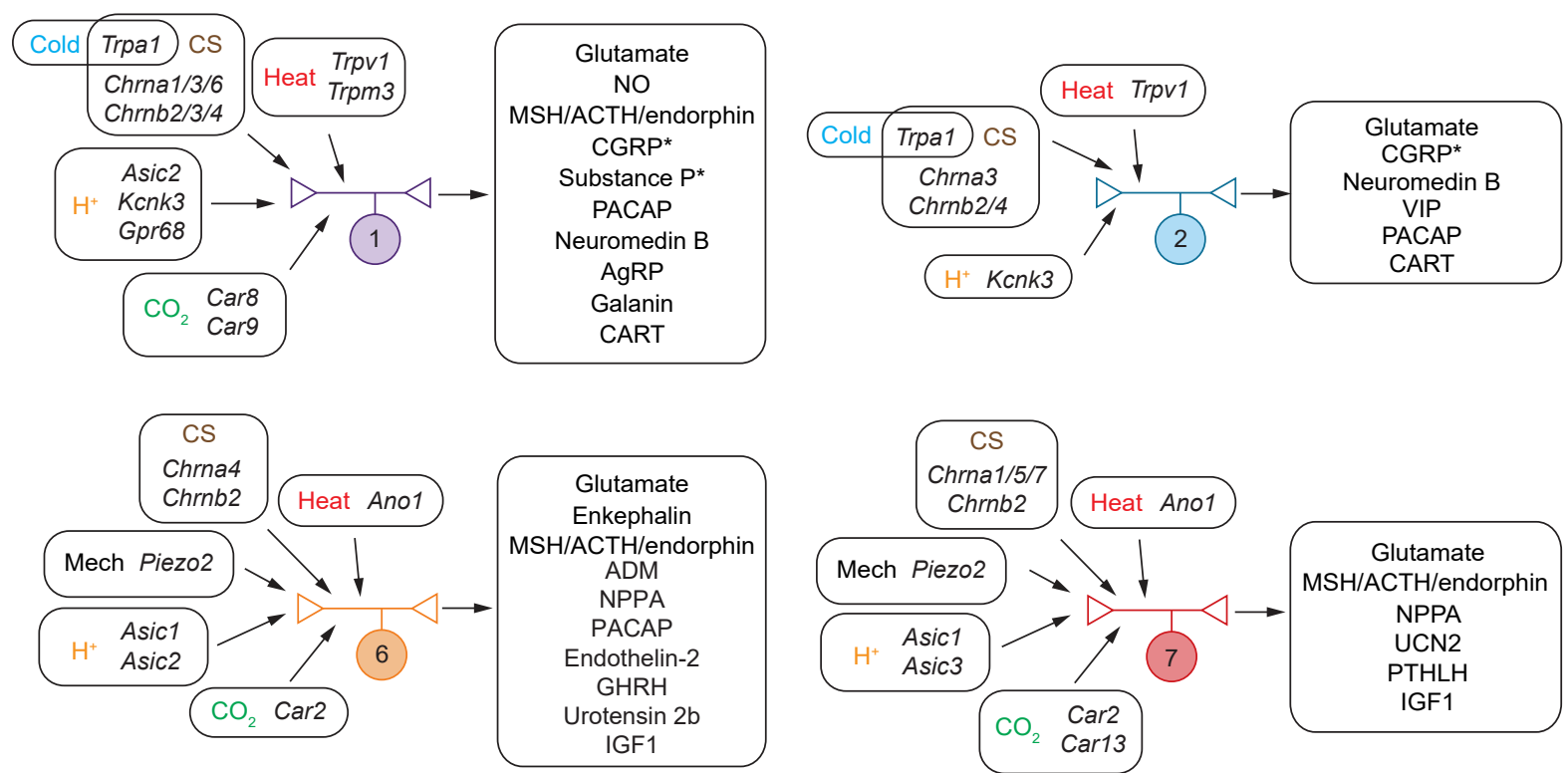
bioRxiv preprint doi: https://doi.org/10.1101/2021.11.10.468116; this version posted November 13, 2021. The copyright holder for this preprint (which was not certified by peer review) is the author/funder. All rights reserved. No reuse allowed without permission.

Figure 4

A

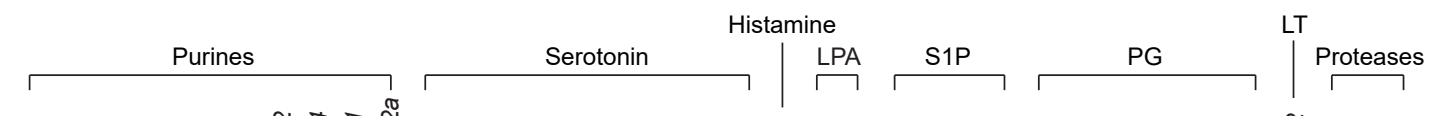

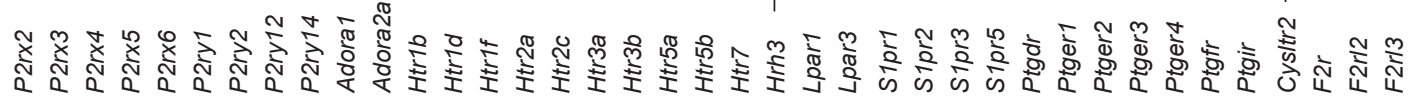

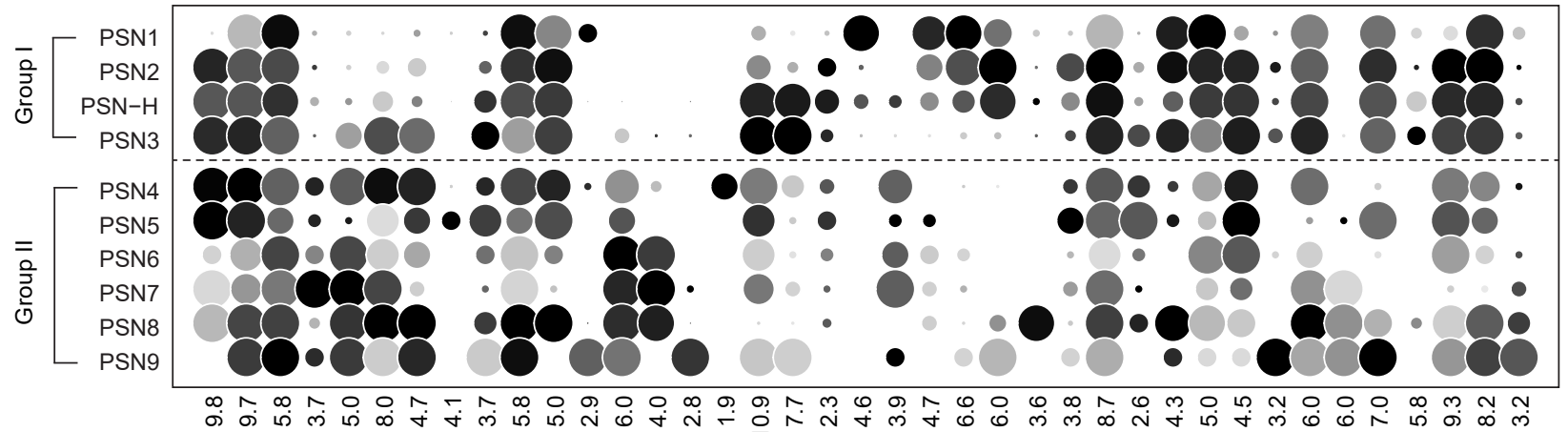

B

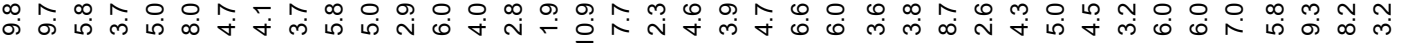
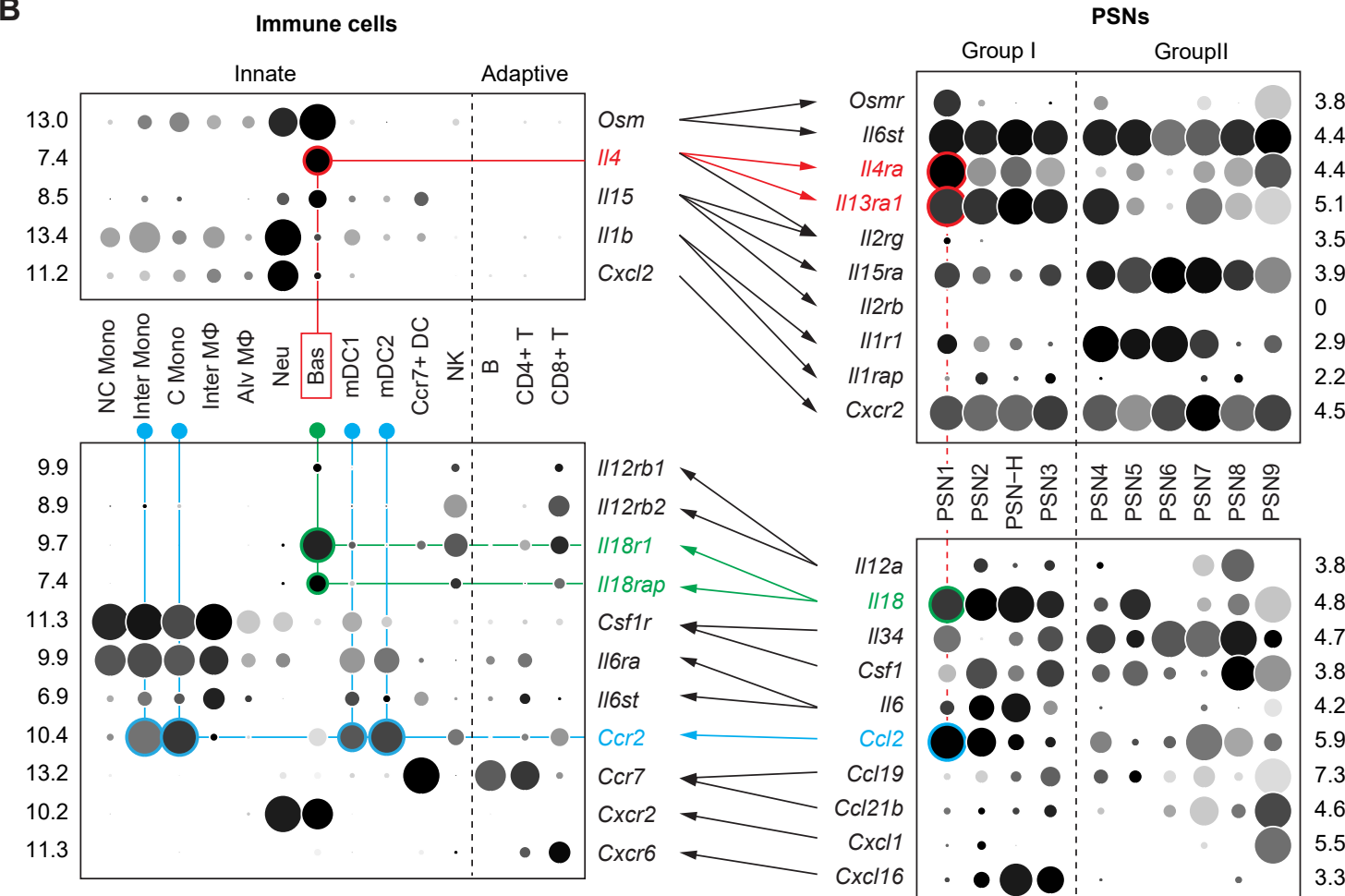

$\%$ of cells
$\circ \quad 25$
$\bigcirc 50$
775
100

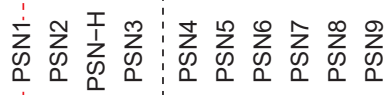

C

Purines, Serotonin, Histamine
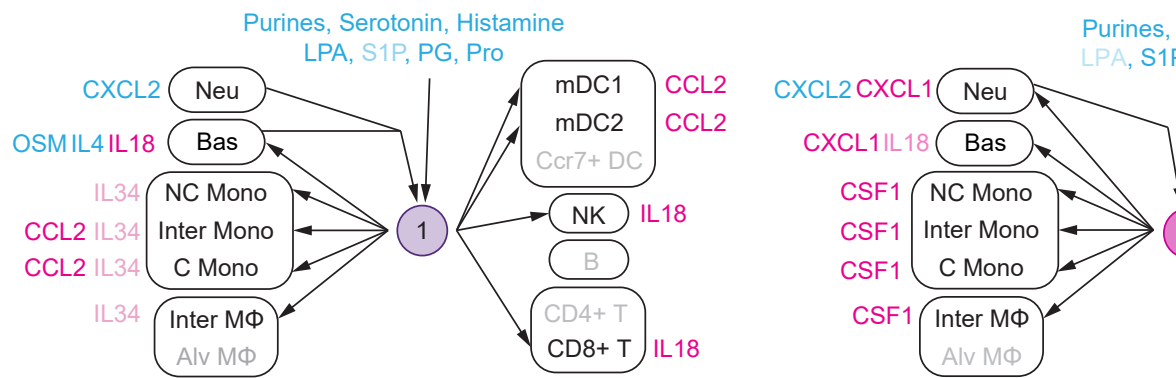

Purines, Serotonin

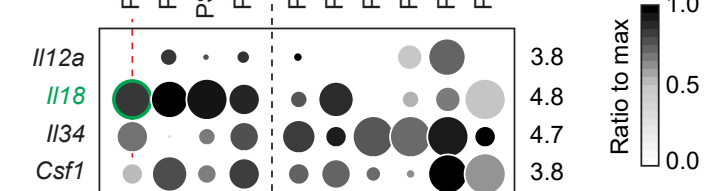

Purines, Serotonin
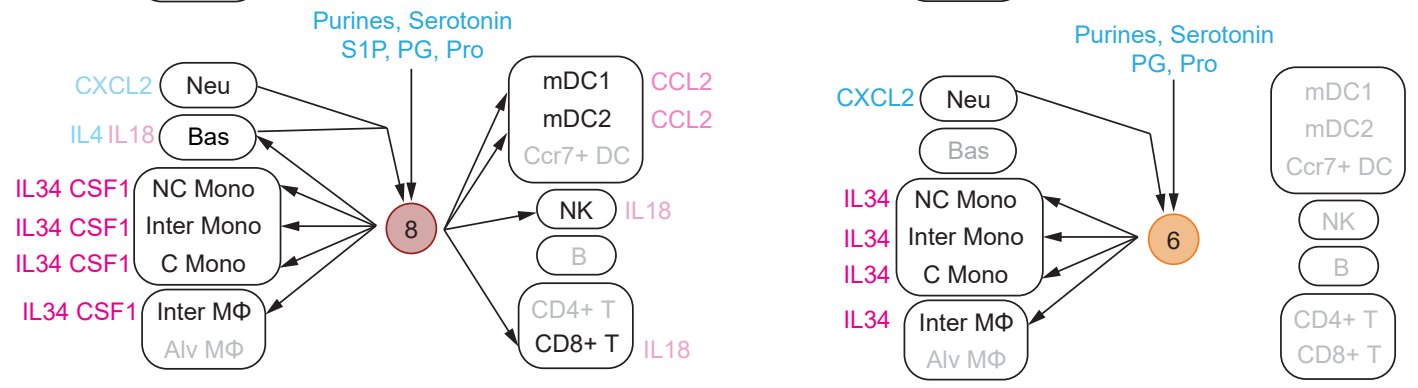
Figure 5

A

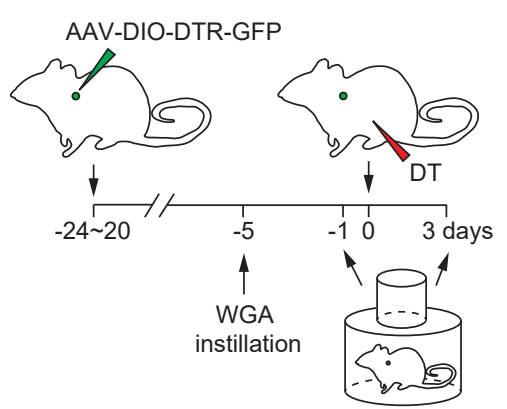

D

PSN6-DTR
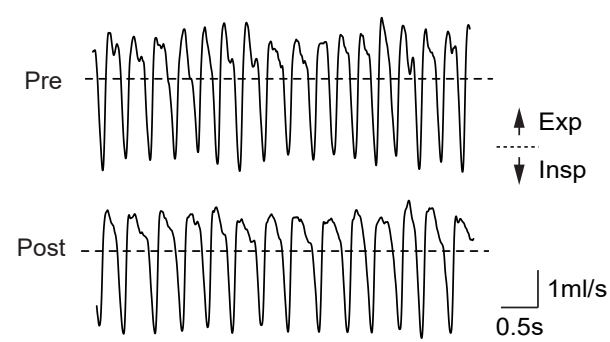

B

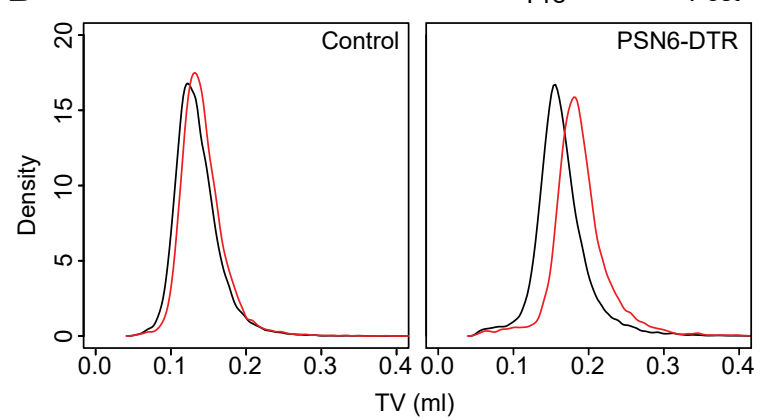

E
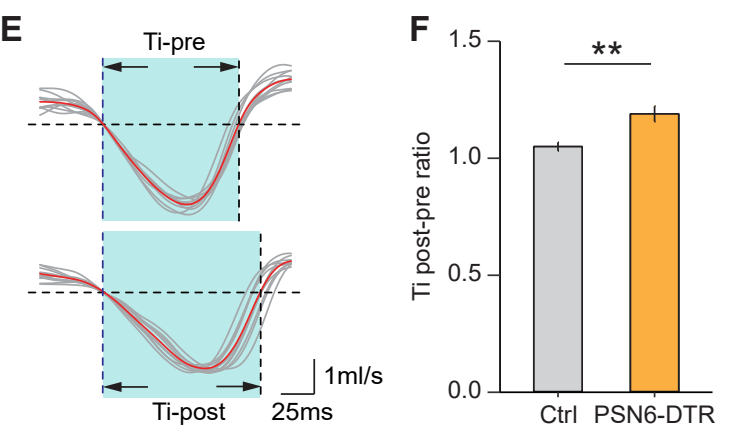
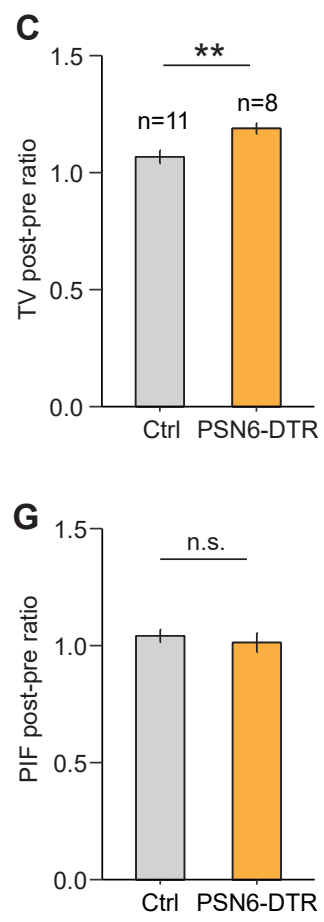

H

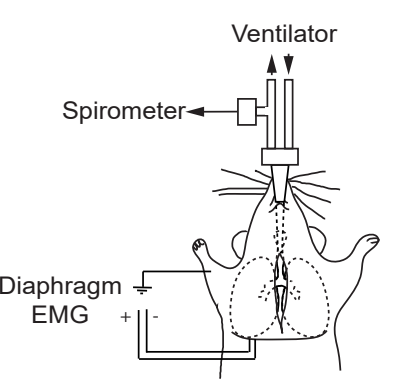

I

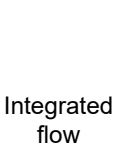

Hold at expiration $\left(2 \mathrm{cmH}_{2} \mathrm{O}\right)$ flow

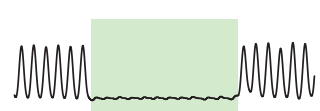

EMG

J

Hold at expiration

Hold at $8 \mathrm{cmH}_{2} \mathrm{O}$
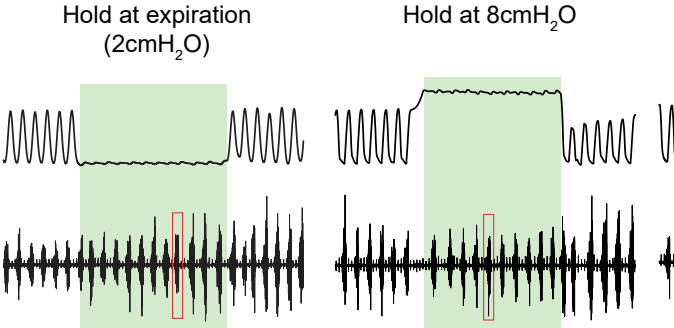

Hold at $10 \mathrm{cmH}_{2} \mathrm{O}$
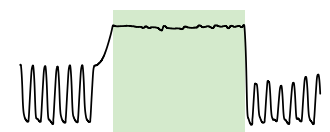

HW

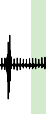

$\sqrt{2}+n^{2}$ $\left(2 \mathrm{cmH}_{2} \mathrm{O}\right)$

Control
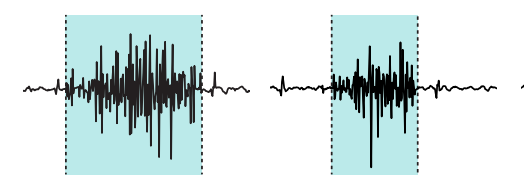

Hold at $10 \mathrm{cmH}_{2} \mathrm{O}$

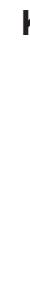

PSN6-DTR
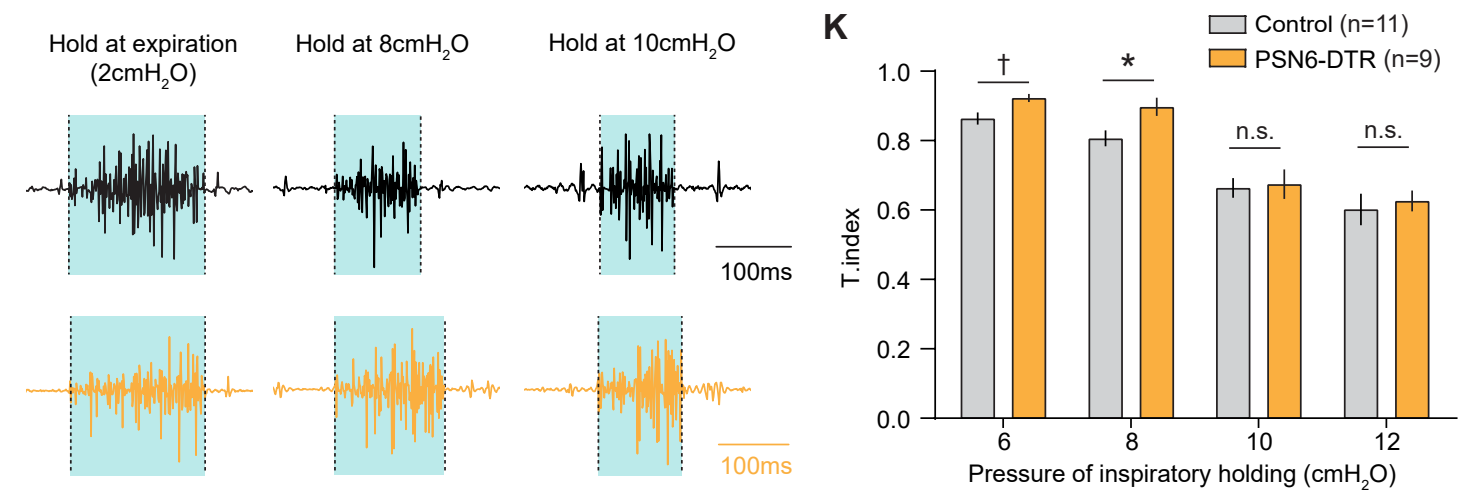


\section{Figure 6}

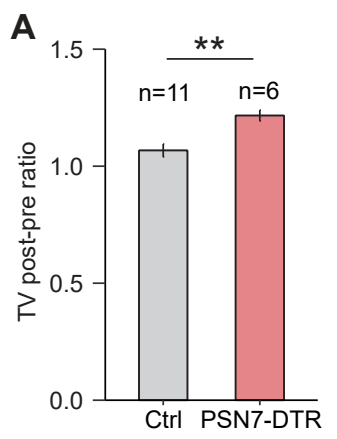

B

PSN7-DTR

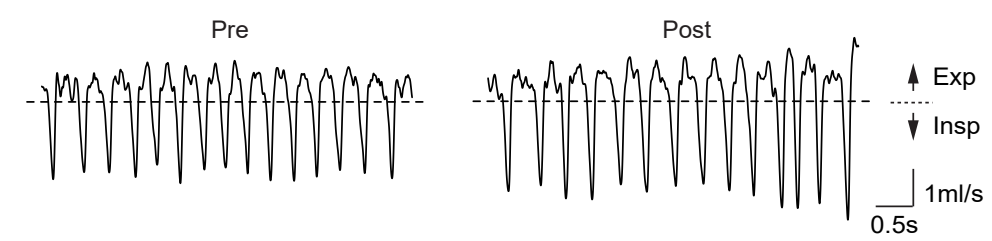

C
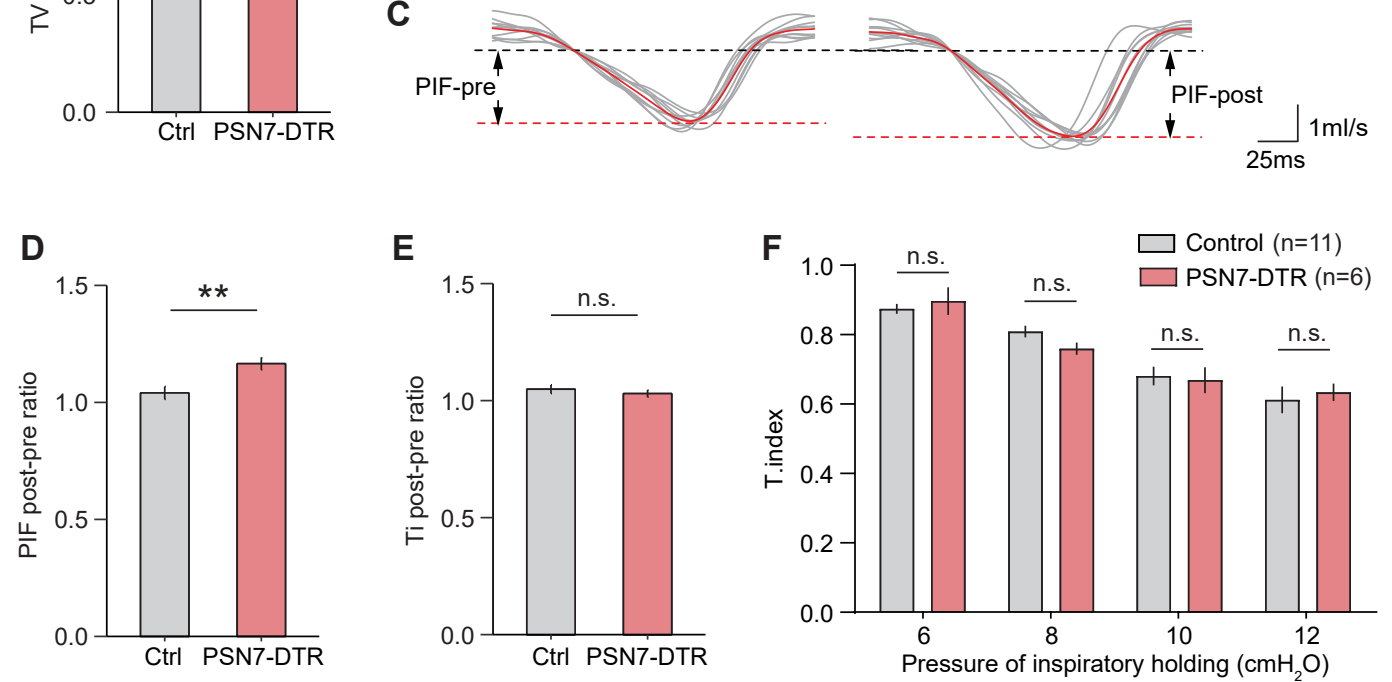
bioRxiv preprint doi: https://doi.org/10.1101/2021.11.10.468116; this version posted November 13,2021 . The copyright holder for this preprint (which was not certified by peer review) is the author/funder. All rights reserved. No reuse allowed without permission.

\section{Figure 7}

A

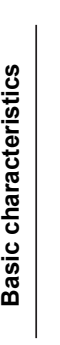

Developmental lineage

Cell Size

Fiber type $(P)$

Conventional

subtypes (P)

Molecular

markers

No. of cells

Thermal

Acids

Irritants

Mechanical

Hypoxia

Hypercapnia

Light

Volatile chem

Bacteria

Inflammation

Neurotransmitters

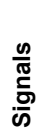

Neuropeptides

Cytokines
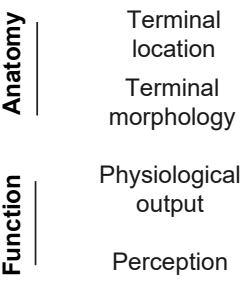

Group I

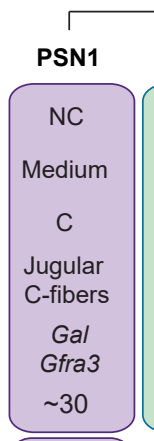

\begin{tabular}{ccc|}
\hline NP & NP & NP \\
Small & Small/ & Small/ \\
Medium & Medium \\
C & C & C \\
\multicolumn{3}{c}{ Nodose C-fibers } \\
Npy2r & \multicolumn{3}{c}{ Htr3b } \\
Rprml & & Mtnr1a \\
& $\sim 200$ &
\end{tabular}

$\operatorname{Trpv1/2}$

Trpm3
Kcnk3
Gpr68
Asic2
Trpa1
Chrna/b

\begin{tabular}{lll}
\hline $\operatorname{Trpv1/2}$ & $\operatorname{Trpv1/2} \quad \operatorname{Trpv2}$ \\
\hline
\end{tabular}

Kcnk3 Kcnk3

Kcnk3 Kcnk3

Asic $2 / 3 \quad$ Asic $2 / 3$

Trpa1 Trpa1

Chrna/b Chrna/b

.

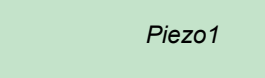

Car8/9

Opn3

Olfr920

TIr4
Hrh3
Lpar1/3
II4ra
Osmr
Cxcr2

\begin{tabular}{ccc} 
Olfr920 & Olfr920 & \\
& & Naip1 \\
Lpar1/3 & Lpar3 & S1pr3 \\
S1pr3 & S1pr3 & Tnfrsf1a \\
Tnfrsf1a & Tnfrsf1a & Cxcr2 \\
Cxcr2 & Cxcr2 & \\
\hline
\end{tabular}

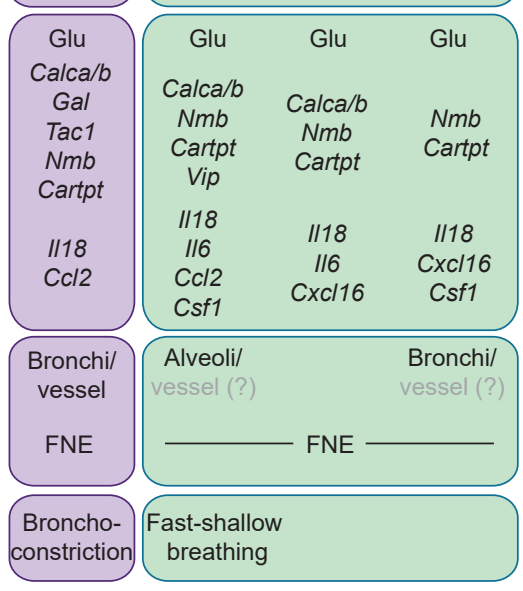
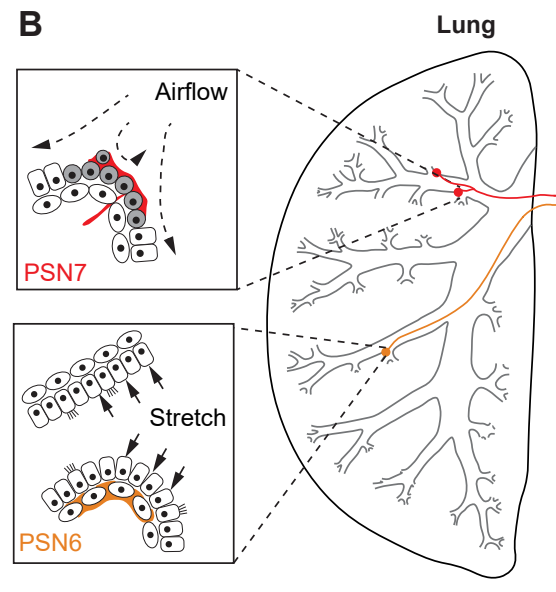

C
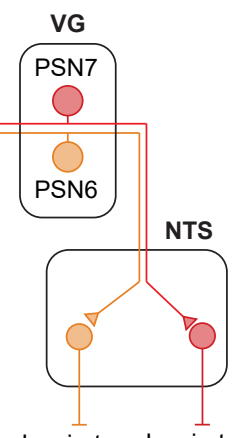

Inspiratory Inspiratory time flow
Group II
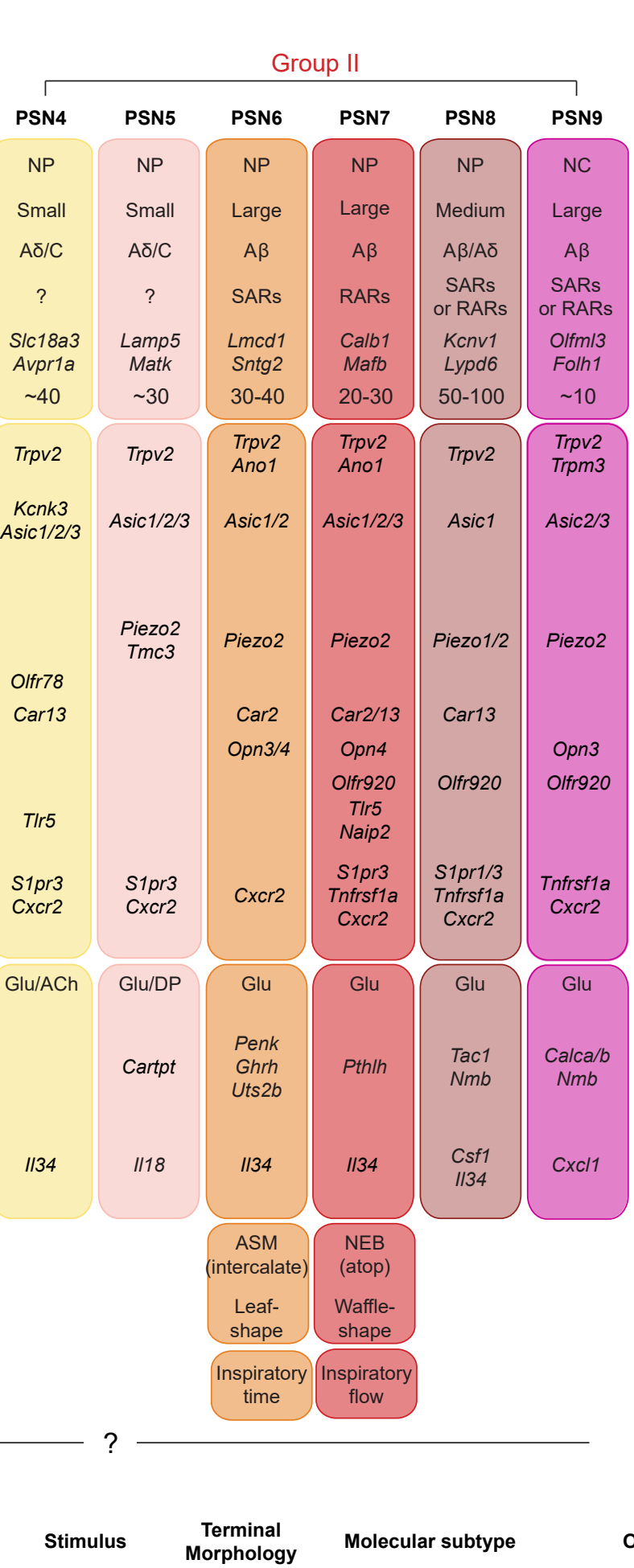

Lung
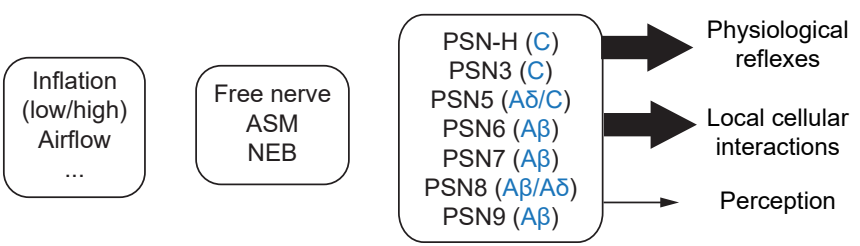

Skin $\begin{gathered}\text { Indentation } \\ \text { Hair deflection } \\ \text { Stroking } \\ \text { Hair pulling } \\ \text { Pricking } \\ \ldots\end{gathered}$
Output

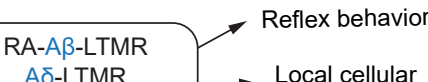
C-LTMR
SA/Field-A $\beta$-LTMR HTMRs $(A \bar{\delta} / \mathrm{C})$
Aঠ-LTMR 


\section{Figure S1}
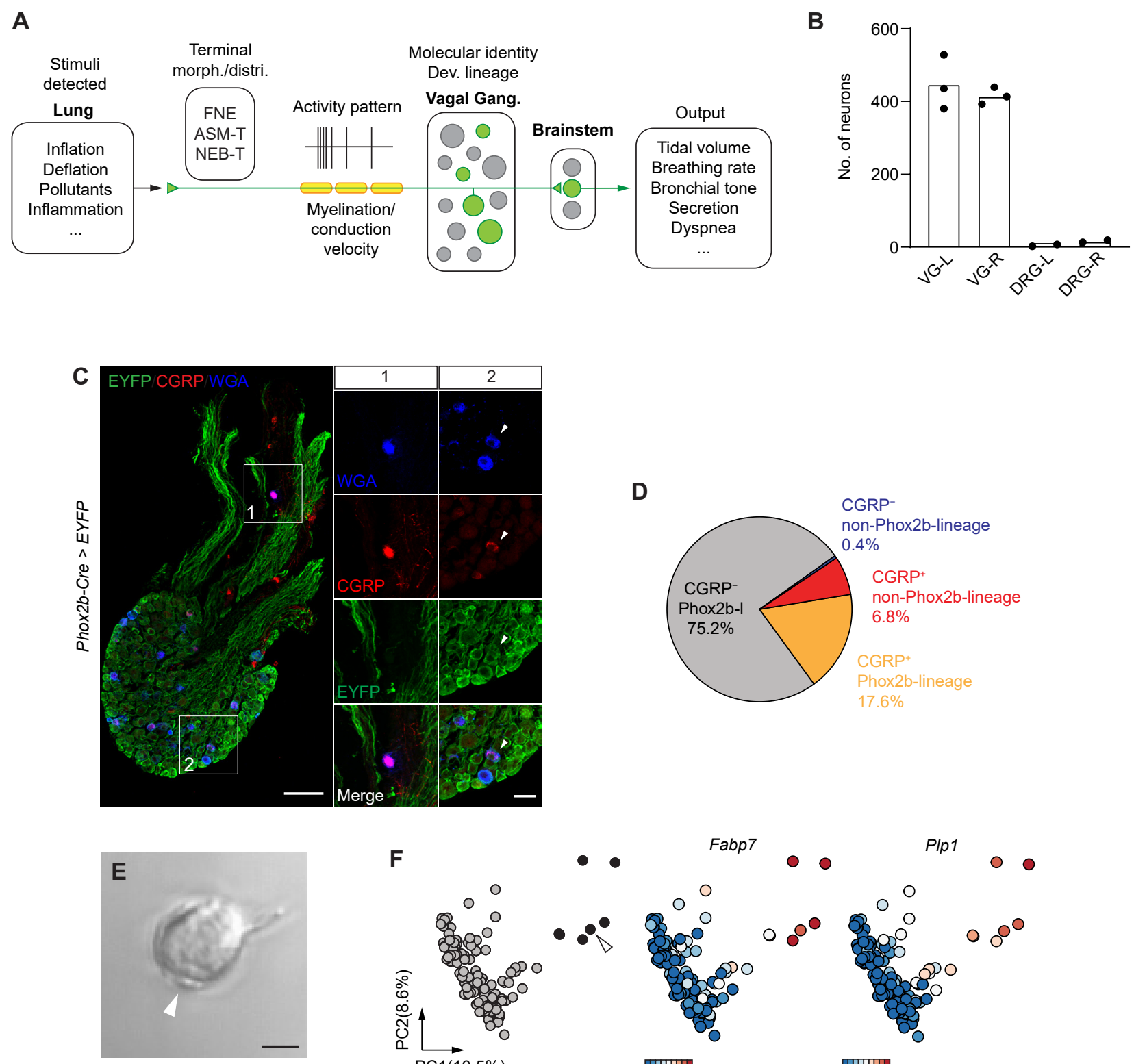

$\mathbf{F}$

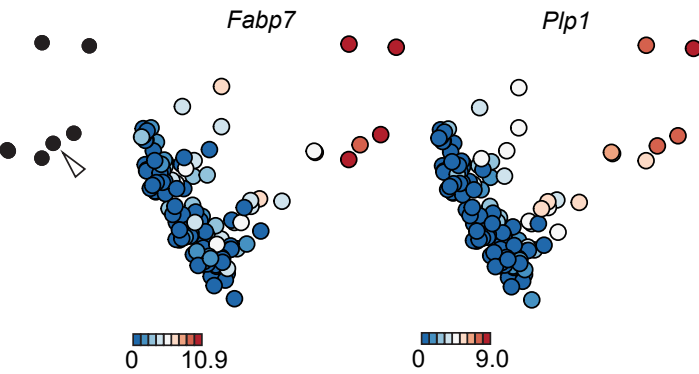

$\mathbf{G}$

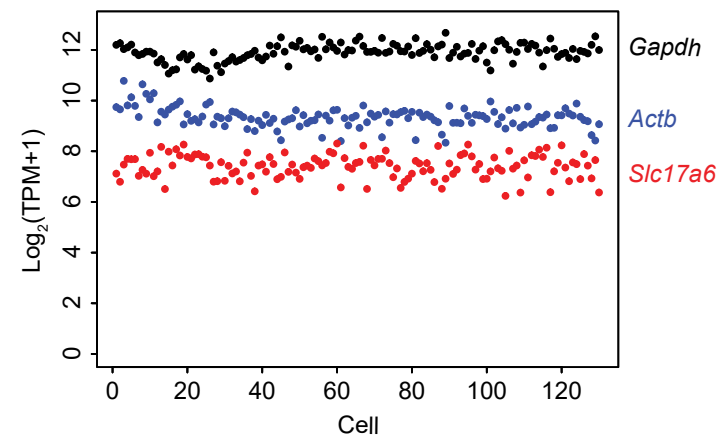

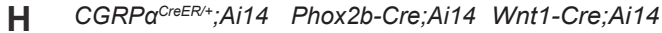

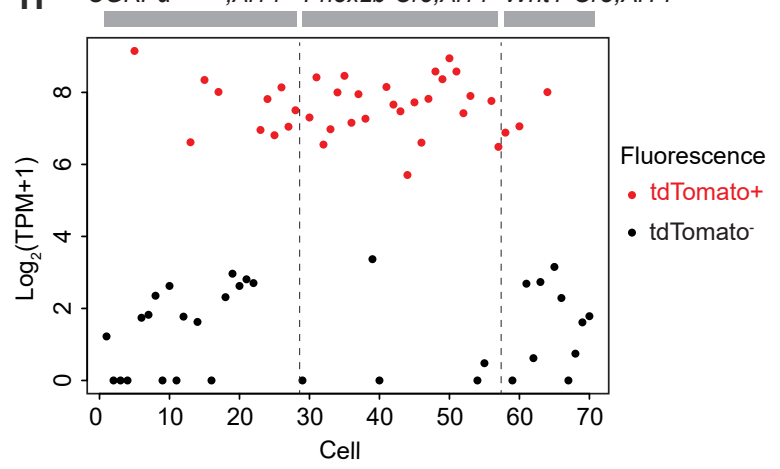




\section{Figure S2}
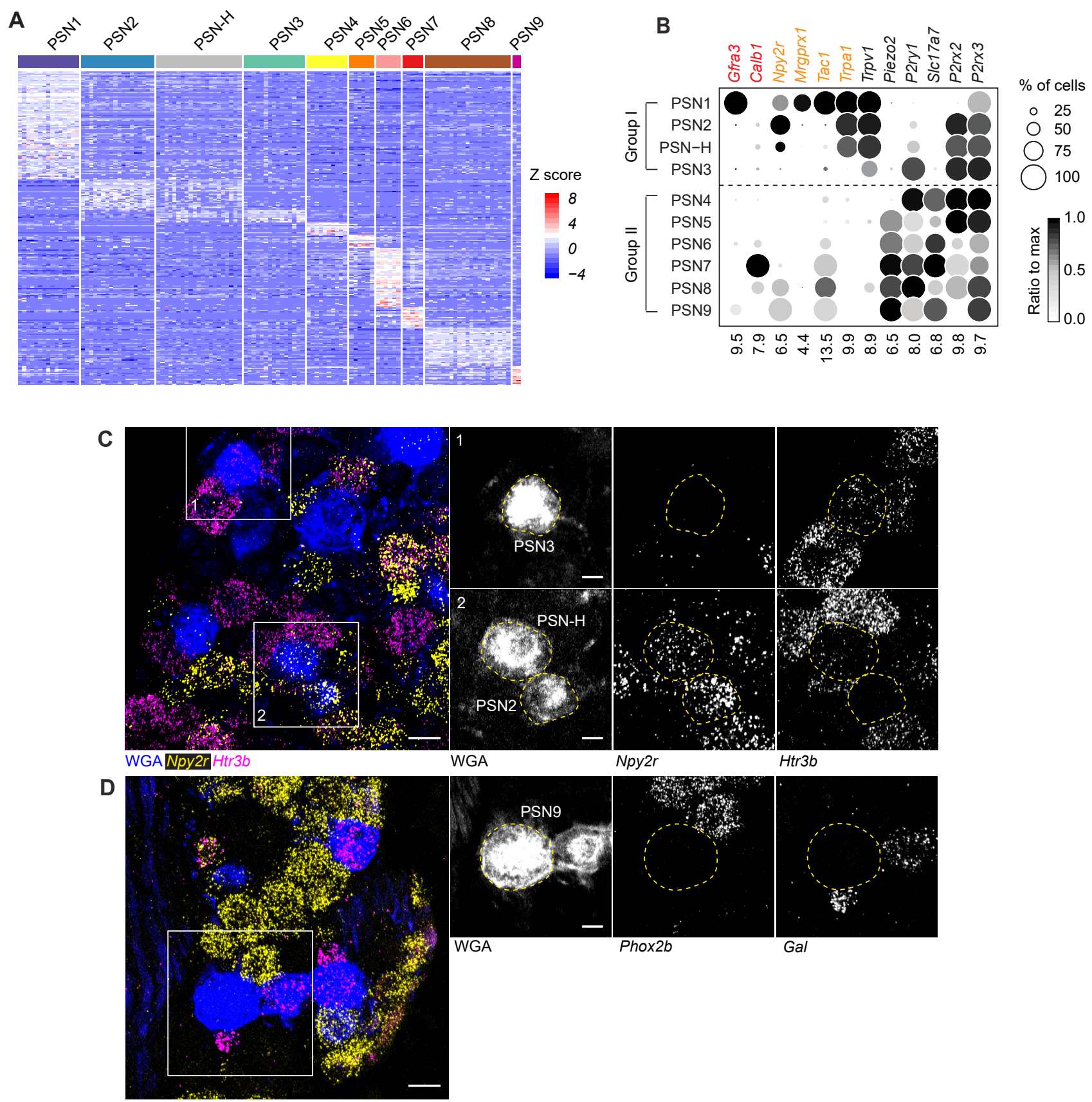

WGAPhox2b Gal

E

\begin{tabular}{ccccc}
\hline Subtype & Genes & No./total WGA+ cells & $\%$ & n (ganglia) \\
\hline PSN1 & Gal+ & $48 / 620$ & 7.7 & 3 \\
PSN2 & Npy2r+Htr3b- & $143 / 772$ & 18.5 & 3 \\
PSN-H & Npy2r+ Htr3b+ & $75 / 772$ & $9.7^{*}$ & 3 \\
PSN3 & Npy2r- Htr3b+ & $166 / 772$ & $21.5^{* *}$ & 3 \\
PSN4 & Slc18a3+ & $67 / 832$ & 8.1 & 5 \\
PSN5 & Lamp5+ & $53 / 856$ & 6.2 & 3 \\
PSN6 & Lmcd+ & $45 / 556$ & 8.1 & 3 \\
PSN7 & Calb1+ & $164 / 2211$ & 7.4 & 5 \\
PSN8 & Kcnv1+ & $94 / 618$ & 15.2 & 3 \\
PSN9 & Gal- Phox2b- & $15 / 620$ & 2.5 & 3 \\
\hline
\end{tabular}




\section{Figure S3}

A

AAV-DIO-reporter gene

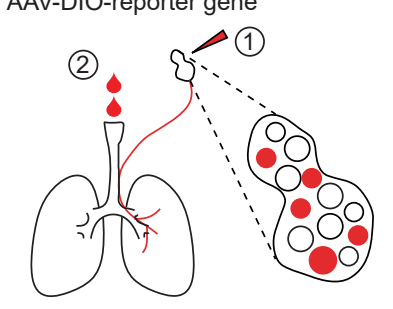

Gene $X \longrightarrow$ Cre
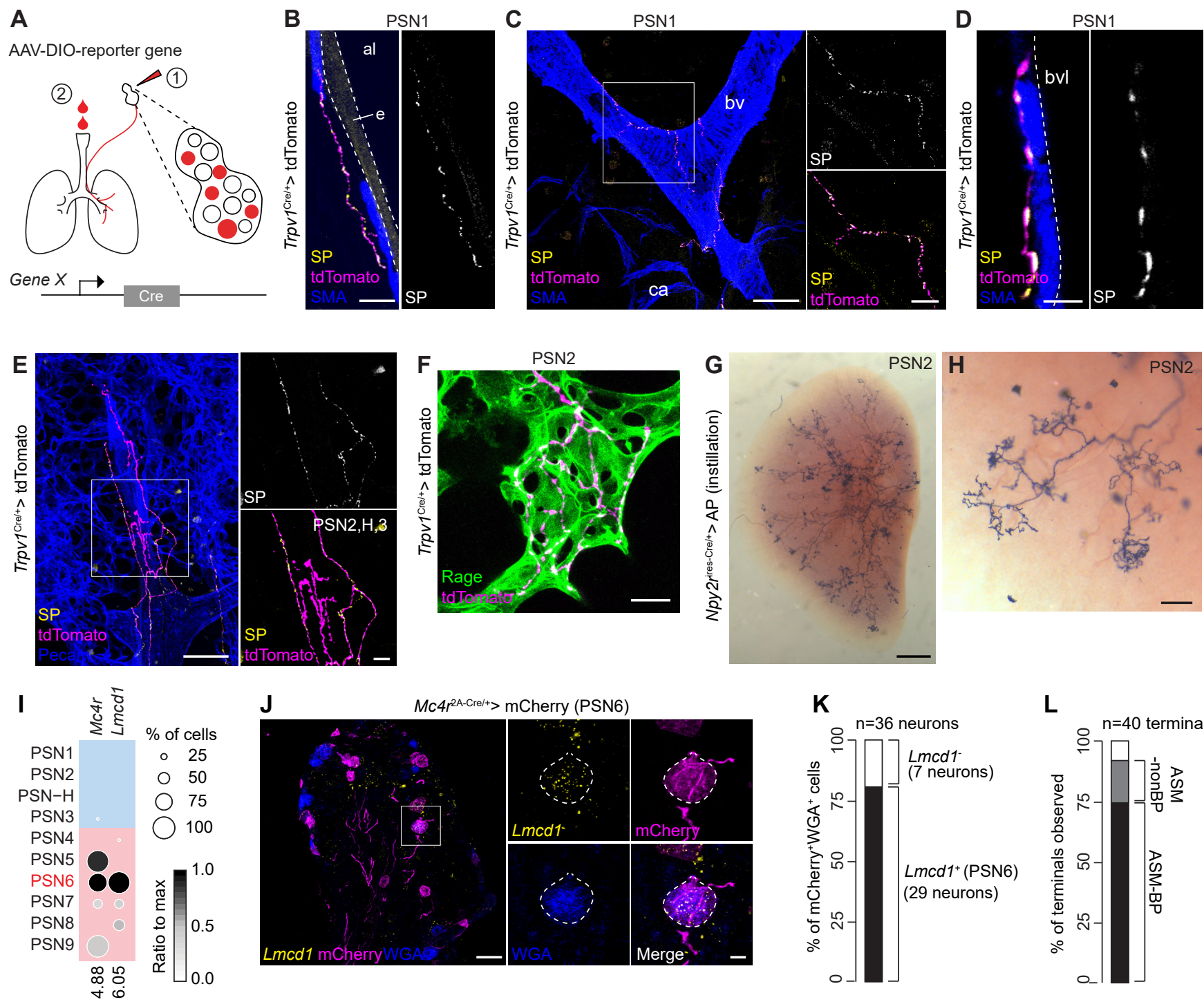

K
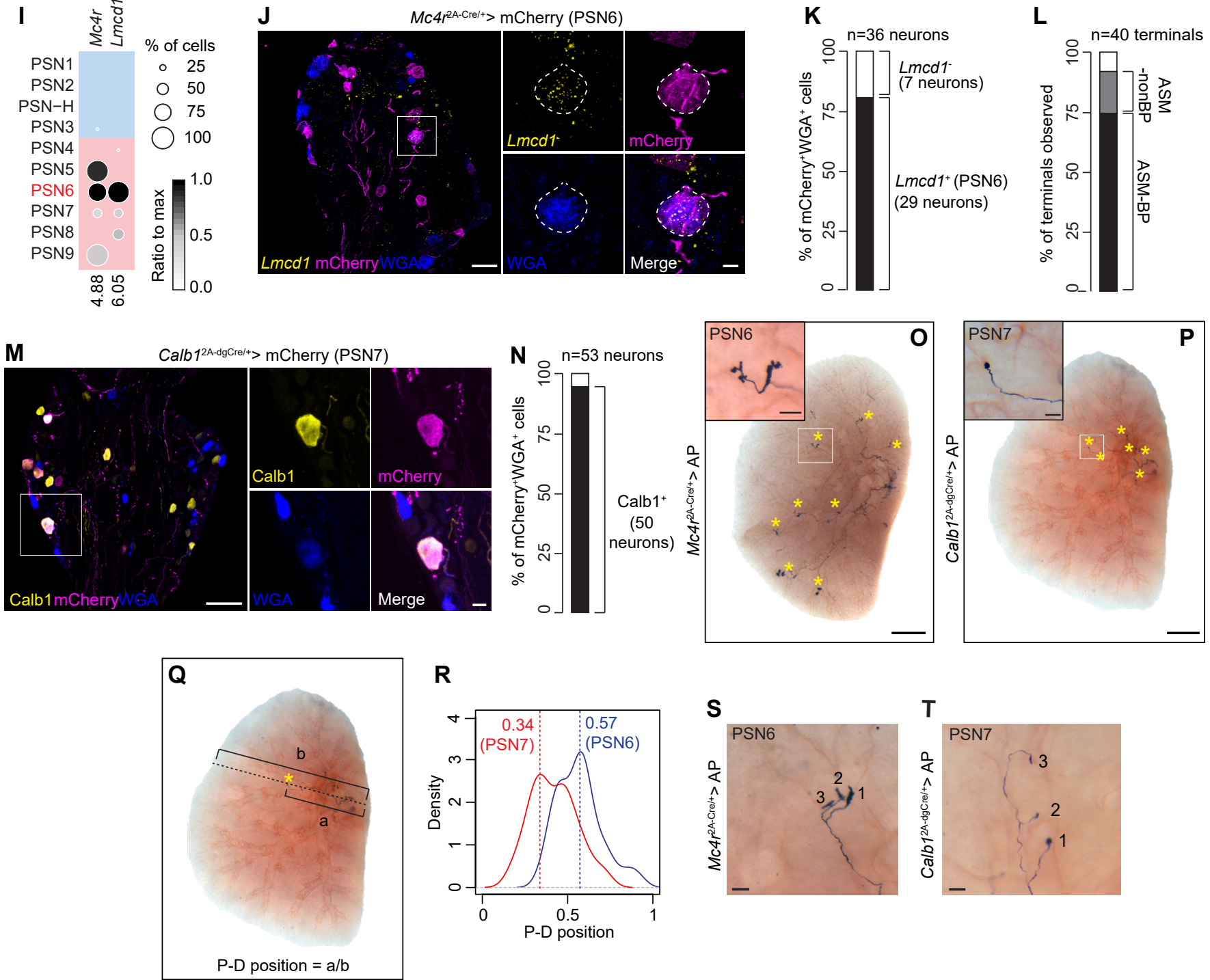

$\mathbf{R}$
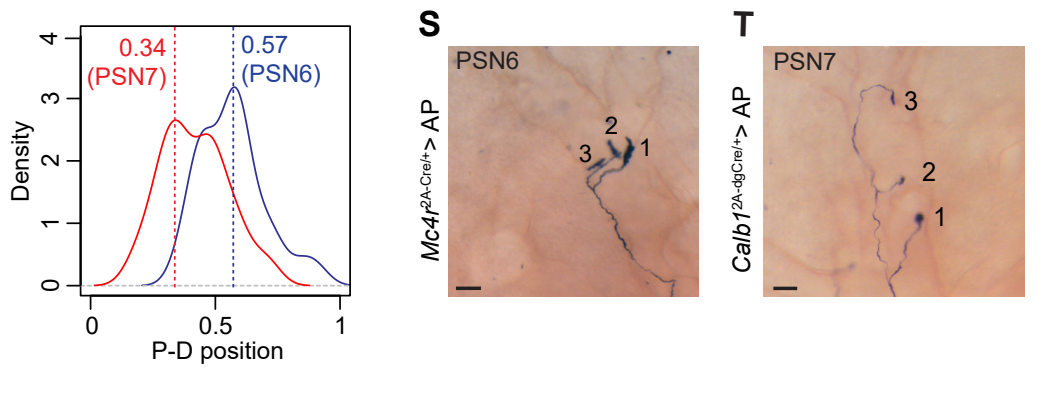
bioRxiv preprint doi: https://doi.org/10.1101/2021.11.10.468116; this version posted November 13,2021 . The copyright holder for this preprint (which was not certified by peer review) is the author/funder. All rights reserved. No reuse allowed without permission.

Figure S4

A

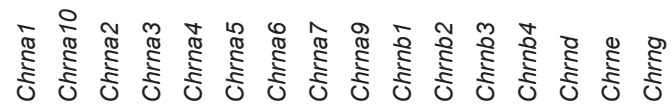

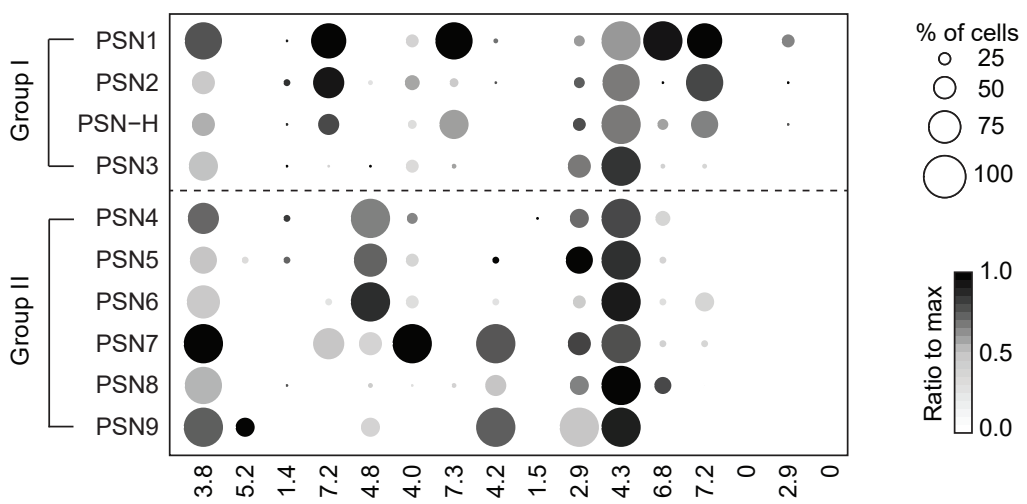

B
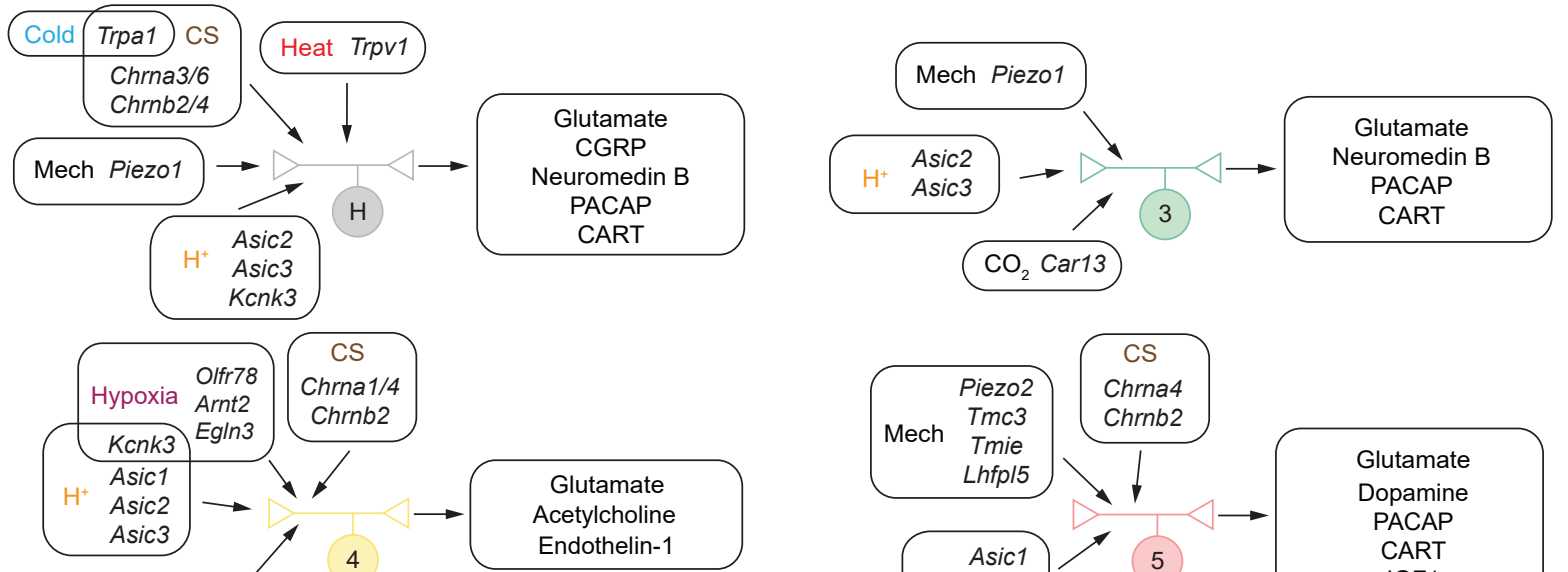

$\mathrm{CO}_{2} \mathrm{Car13}$
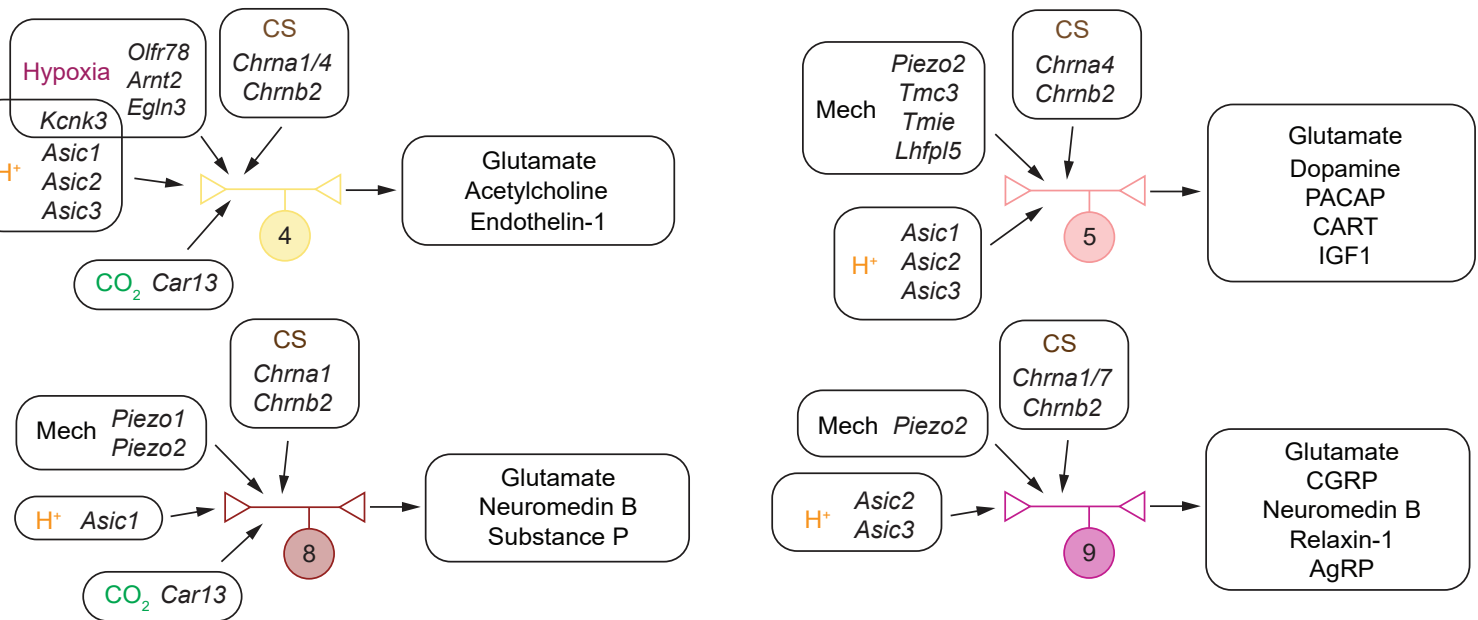

C

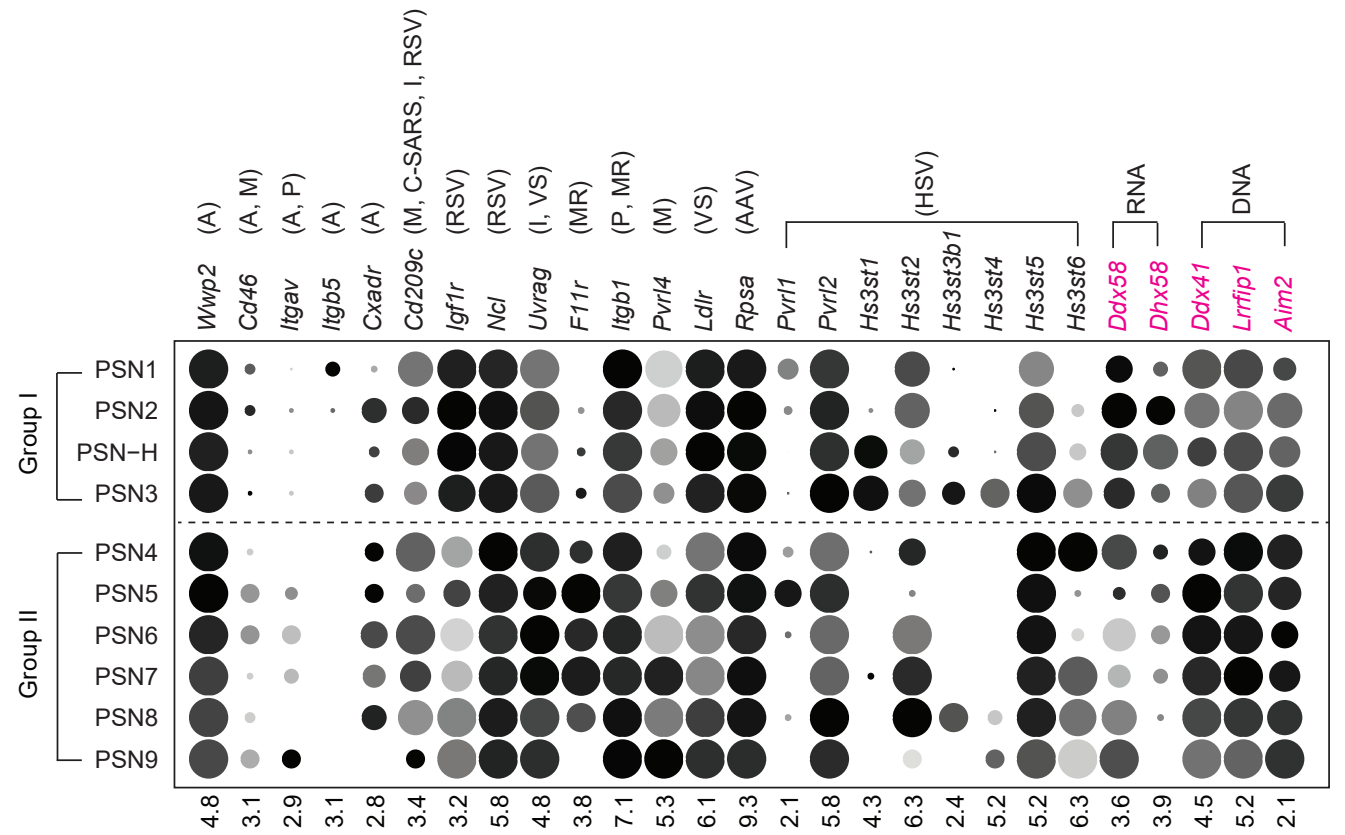


bioRxiv preprint doi: https://doi.org/10.1101/2021.11.10.468116; this version posted November 13, 2021. The copyright holder for this preprint (which was not certified by peer review) is the author/funder. All rights reserved. No reuse allowed without permission.

Figure S5

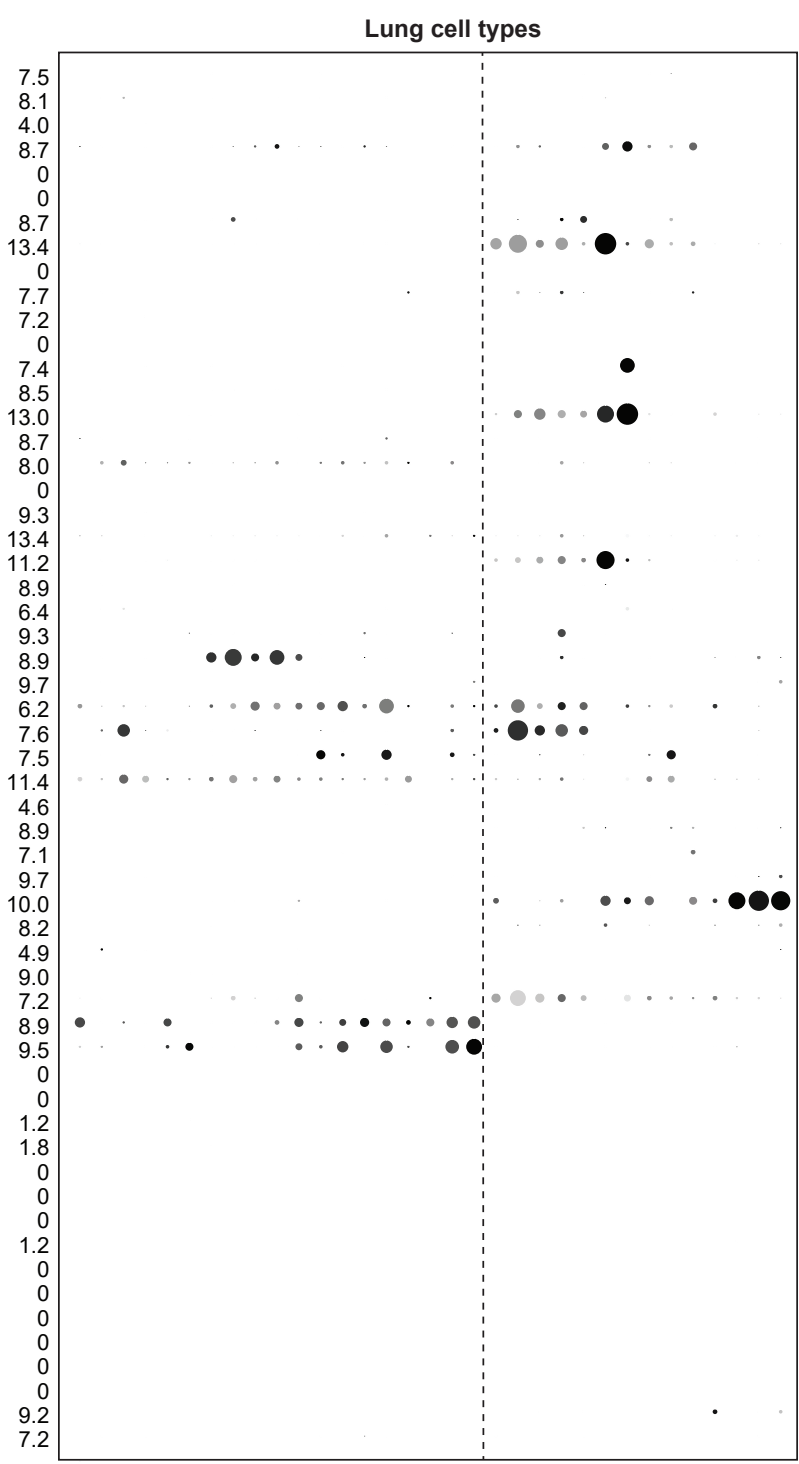

Cytokines Receptors

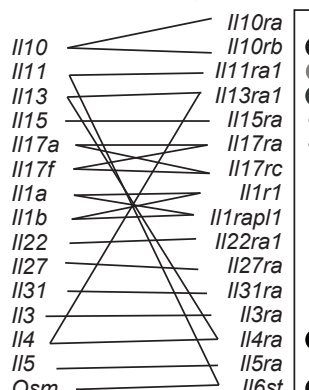

Osm $116 s t$

Ccl27a $\longrightarrow$ Lifr

$\mathrm{C} / 27 b \longrightarrow$
$\mathrm{C} / 28 \longrightarrow C \mathrm{cr} 10$

$\mathrm{Cxcl1} \longrightarrow \mathrm{C}$ Cr10

$\mathrm{Cxcl} 2={ }_{\mathrm{CxCl}} \longrightarrow \mathrm{CxCr} 2$
$\mathrm{CxCr} 5$

$\mathrm{CxCl5}$

Cxcl13 Tnfrsf10b

Tnfsf10 Tnfrsf10b

Tnfsf11 $\square$ Tnfrsf12a

Tnfsf12 $\longrightarrow$ Tnfrsf13b

Tnfsf13b Tnfrsf13c

${ }_{\text {Tnfsf18 }}^{\text {Camfrsf18 }}{ }_{\text {Tnfrsf1a }}$

\begin{tabular}{l|l} 
Tnf \\
Tnfsf 4 \\
Tnfrsf1b 22 \\
Tnfrsf23
\end{tabular}

Lta $\longrightarrow$ Tnfrsf23

Ltb
Tnfsf14
$L t b r$

Csf2 Csf2ra

Tgfb1 $\angle$ Tgfbr1

Tgfb2 $>\quad<\begin{aligned} & T \text { Tgfbr2 } \\ & T \text { Tgfbr3 }\end{aligned}$

Tgfb3

Ifna1

Ifna11

Ifna12
Ifna13
Ifna14

Ifna2

Ifna2
Ifna4
Ifna5

Ifna6

Ifna7

Ifna9

Ifnab

Ifne

Ifng
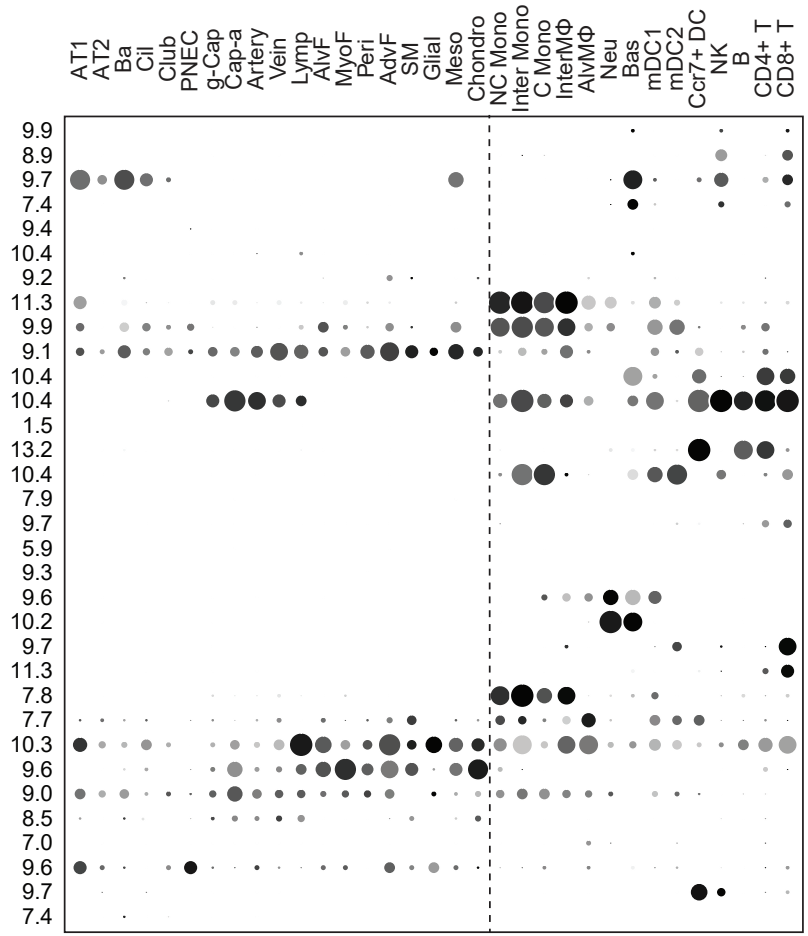

Receptors Cytokines

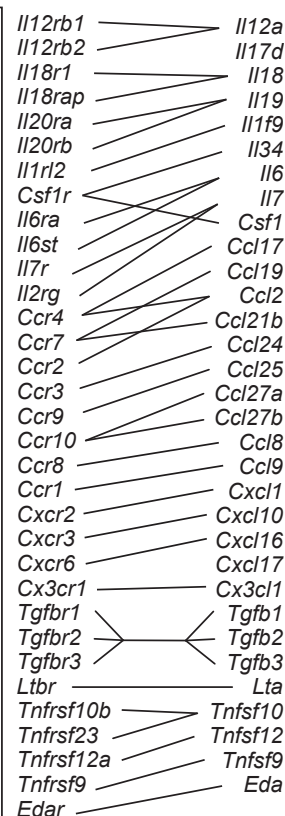

PSNs

$0000000^{2}{ }_{7.7}^{2.8}$

0000989007.7

000:- 5.1

- 000003.9

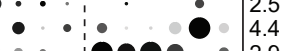

- . 0000.2 .9

• .

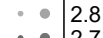

000

-

10000004.4

100

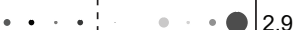

00000080
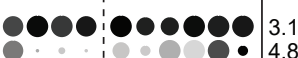

00000

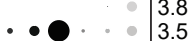

$000 . .003 .4$

000 :

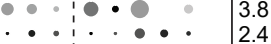

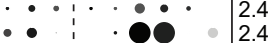

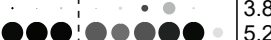

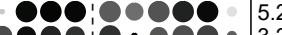

... - 0000

$10 \bullet \cdot 0 \cdot 00003.9$

••..$\bullet 2.0$

 (808):80888 5.1 (5.3

6.8

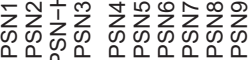

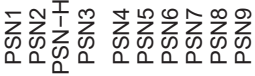

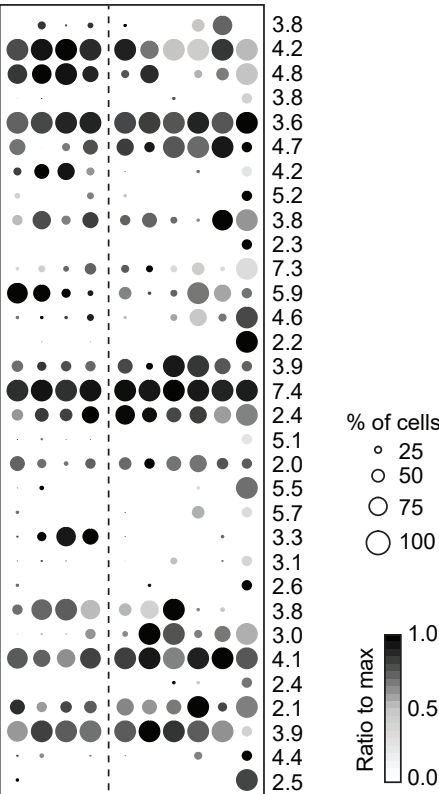




\section{Figure S6}
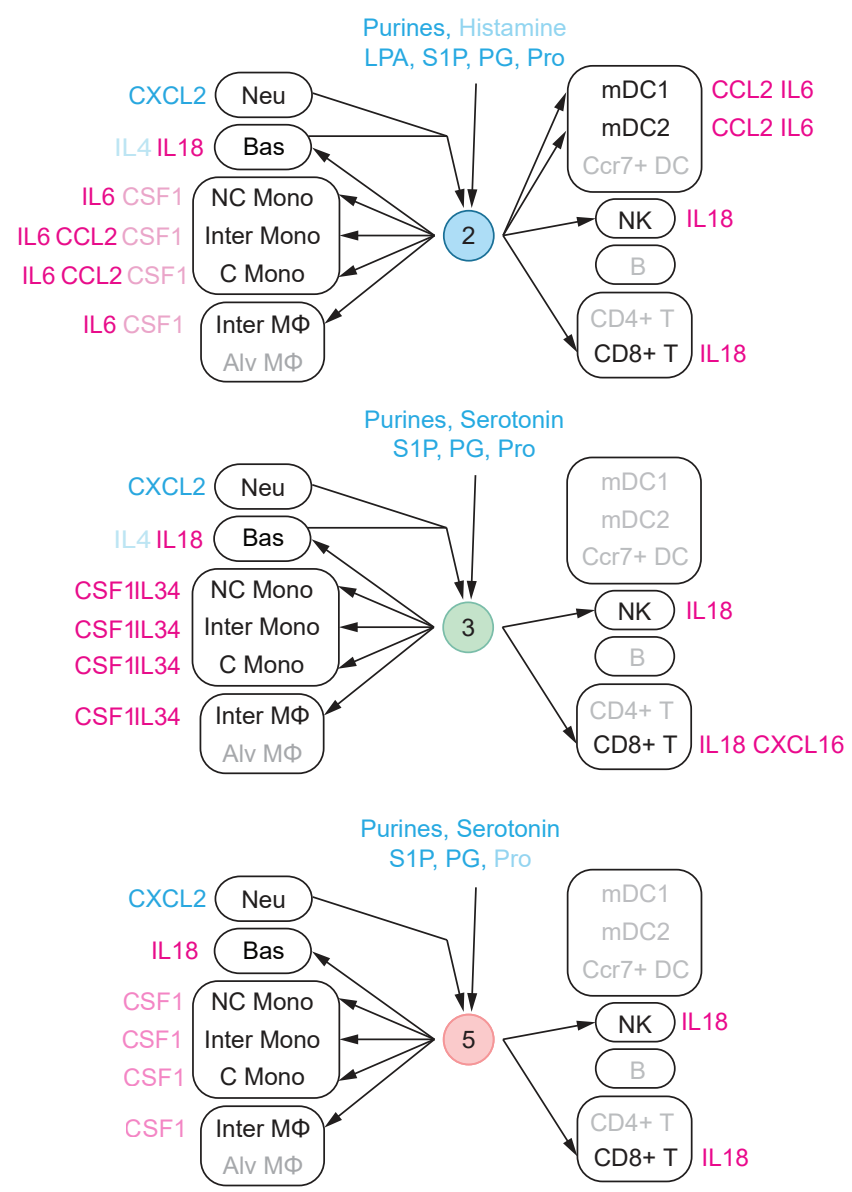
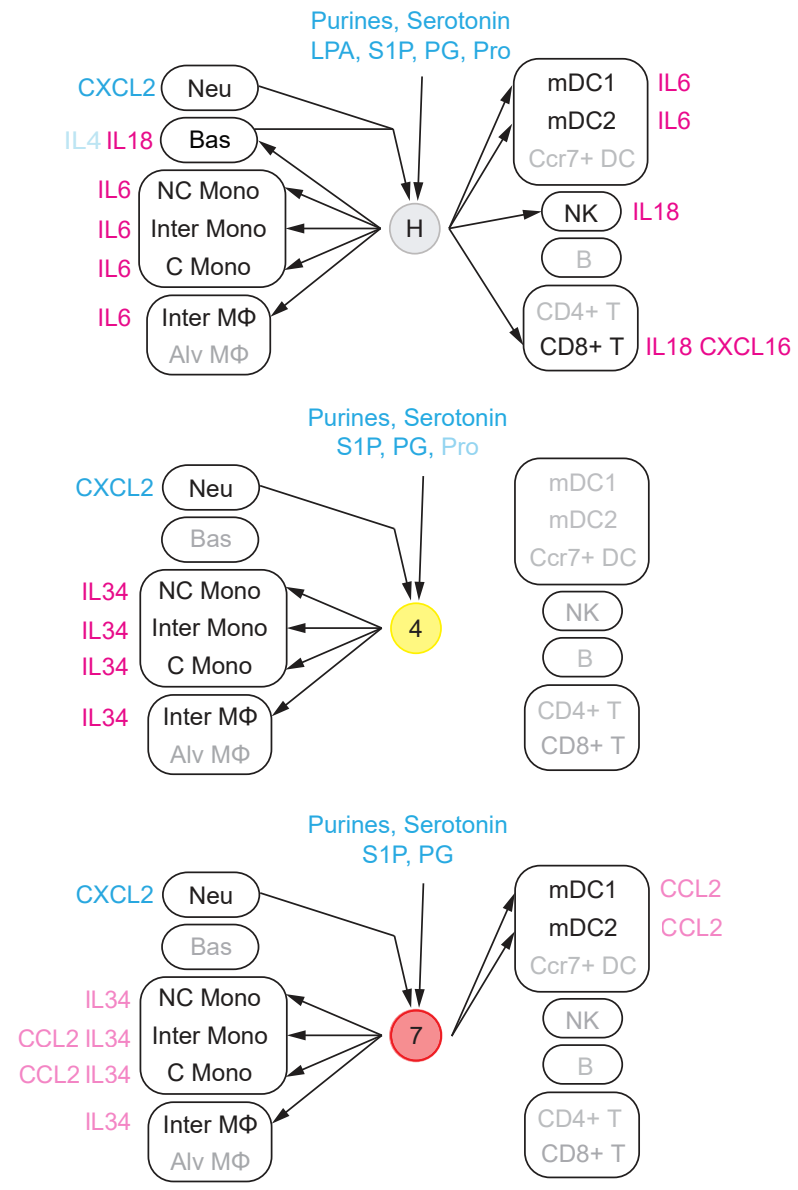
Figure S7
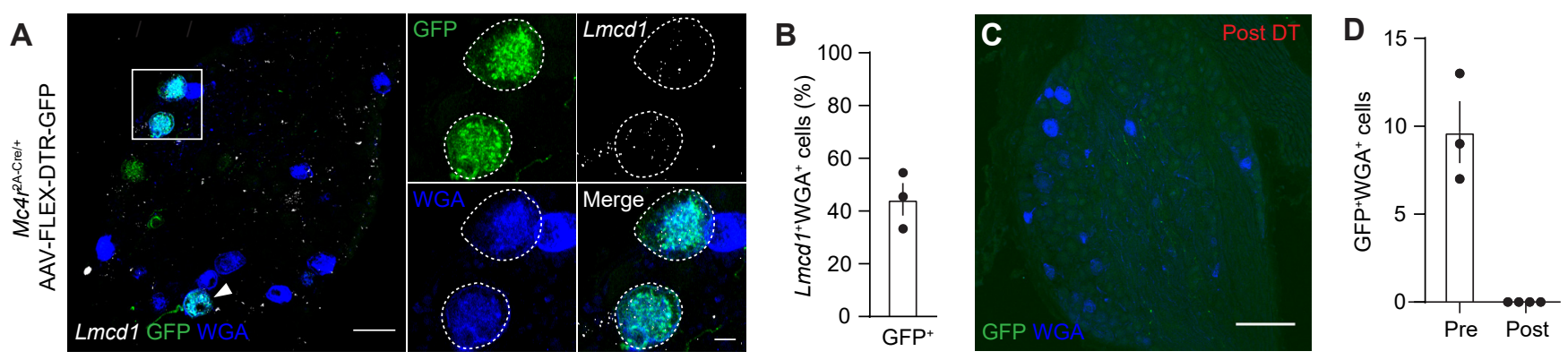

E

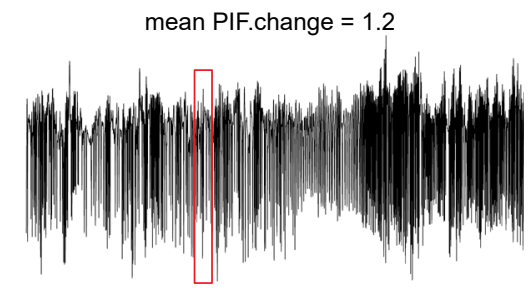

Exp
Quiet

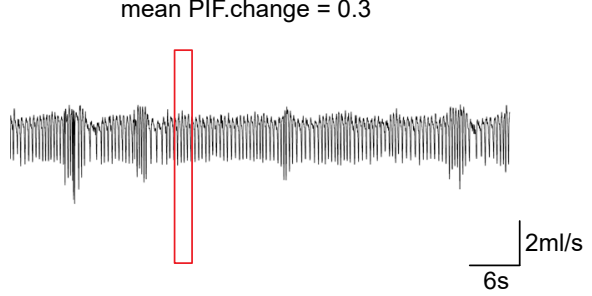

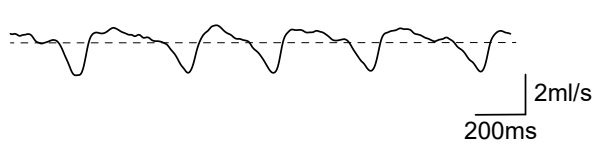

$\underset{200 \mathrm{~ms}}{2 \mathrm{~m} / \mathrm{s}}$

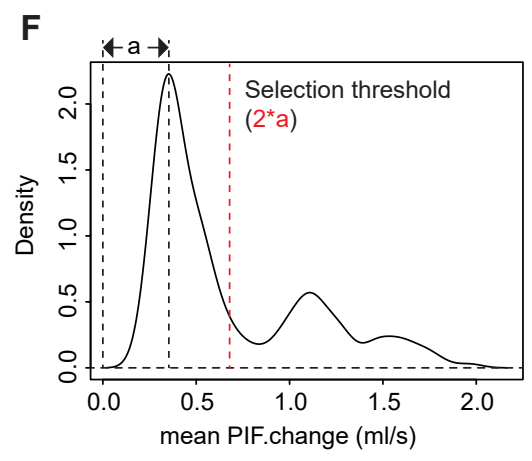

breath.1
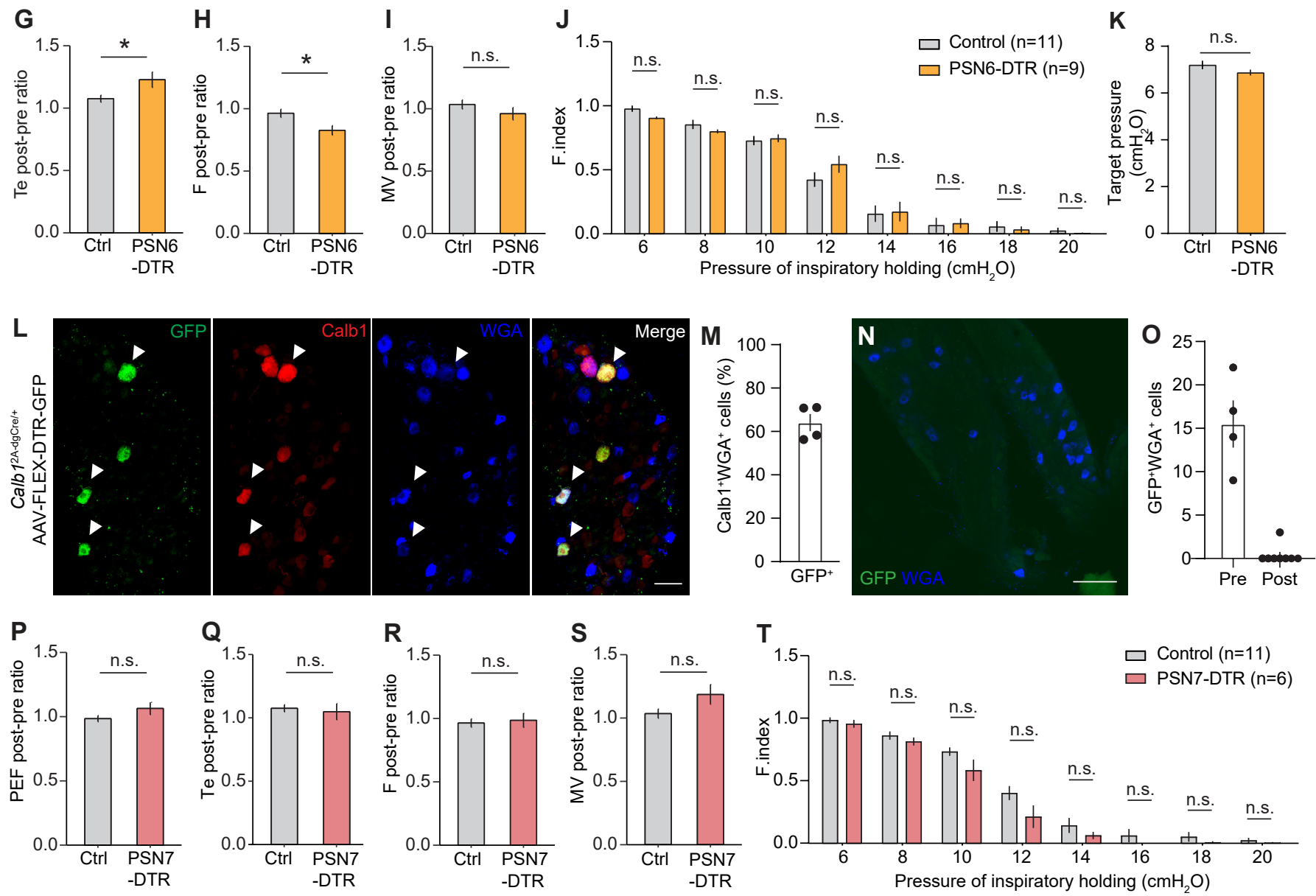